\title{
The Geometry of the Milnor Number
}

\author{
Dissertation
}

\author{
zur Erlangung des \\ mathematisch-naturwissenschaftlichen \\ Doktorgrades \\ "Doctor rerum naturalium" \\ der Georg-August Universität Göttingen
}

\author{
vorgelegt von \\ Adrian Szawlowski \\ aus Berlin
}

Göttingen 2012 
Referent: Prof. Dr. Victor Pidstrygach Koreferent: Prof. Dr. Thomas Schick

Tag der mündlichen Prüfung: 19. April 2012 
For my family 



\section{Acknowledgements}

Ich danke meiner Familie, besonders meinen Eltern, für eure Unterstützung und Liebe in all den Jahren und meinen Freunden, die ihr mir das Leben lebenswert gemacht habt.

I also like to thank my advisor Victor Pidstrygach for freeness in my research and very nice discussions during tea time. Last but not least I am thankful to M. Garay, J.-P. Françoise and Lê D. T. for useful conversations. 


\section{Introduction}

What is a singularity? In agreement, both the Star Trek community and researchers in differential geometry will understand it as a point in space of infinite curvature. However there is really no need to go into spacetime to find these things. Quite often during the last year when I looked into my wallet I discovered a singularity, too. In this thesis we will however be concerned with those singularities that occur in local analytic geometry.

It is an old habit of mathematicians to find hopefully interesting group actions and look at the orbits. Let's do that. If $(M, \omega)$ is a symplectic manifold then the group $\operatorname{Sp}(M, \omega)$ of symplectomorphisms has an adjoint action. Concretely it acts on the vector space of symplectic fields $\mathfrak{g}=T_{i d} \operatorname{Sp}(M, \omega)=$ $\left\{X \in \mathbb{C}^{\infty} T M \mid L_{X} \omega=0\right\}$ by

$$
\begin{aligned}
G \times \mathfrak{g} & \rightarrow \mathfrak{g} \\
\operatorname{Ad}_{\phi}(X) & =d_{i d}\left(\operatorname{conj}_{\phi}\right)(X) .
\end{aligned}
$$

If $M$ is connected and 1-connected, then the symplectic fields are exactly the hamiltonian fields which themselves correspond exactly to functions on $M$ modulo constants. In these terms the adjoint action can also be expressed as

$$
\operatorname{Ad}_{\phi}\left(X_{f}\right)=X_{f \circ \phi^{-1}} .
$$

So the problem of finding the orbits comes down to right equivalence of functions modulo constants. What about the manifold $M$ ? We can choose it to be the whole of $\mathbb{C}^{2}$ for example. Such global right equivalence is usually much more difficult to understand than if we just take $M$ to be a very small set, namely a set germ in $\mathbb{C}^{2}$. Note finally that in dimension two, being symplectic or volume-preserving are equivalent conditions. In higher dimensions symplectic is strictly stronger. 


\section{Introduction}

We assume more general that we deal with a holomorphic function germ with an isolated critical point at the origin of $\mathbb{C}^{n}$. Then the most important invariant of right equivalence is the Milnor number. There are several equivalent definitions of this $\mu$ to which we will come in chapter one. One definition is for example

$$
\mu(f)=\operatorname{dim}_{\mathbb{C}} \frac{\mathbb{C}\left\{x_{1}, \ldots, x_{n}\right\}}{\left\langle\partial_{1} f, \ldots, \partial_{n} f\right\rangle} .
$$

Volume-preserving geometry leads to the interpretation of $\mu$ in connection with the Brieskorn module

$$
H_{f}^{\prime \prime}=\frac{\Omega_{\mathbb{C}^{n}, 0}^{n}}{d f \wedge d \Omega_{\mathbb{C}^{n}, 0}^{n-2}}
$$

whose rank is also $\mu(f)$. Their relationship will be considered in chapter two. A third interpretation of $\mu$ occuring in the theory of unfoldings has led me to a formula which is nested in the realm of pencils of holomorphic germs. This is the content of chapter three. It is interesting to note that there is another link to symplectic geometry, for we will see that $f_{x} g_{y}-f_{y} g_{x}$ plays an important role in chapter three and this expression is nothing else than the Poisson bracket.

Let us now describe in more detail the various sections and the main results of the thesis.

Chapter one serves as an introduction to singularity theory. All of the material here is "well-known". We give some basic properties of singularities in the first section and in the next section we clarify the situation around the fibration theorems. In particular these two sections will provide us with many definitions of the Milnor number. We will not only deal with a germ $f:\left(\mathbb{C}^{n}, 0\right) \rightarrow(\mathbb{C}, 0)$ but also with higher dimensional target space. These isolated complete intersections will be needed when we look at deformations of the map $f$ in chapter two.

The second chapter deals with volume-preserving geometry. The first section gives a very nice overview of the situation, so I recommend to read it as well as this introduction. The complex version of the Morse lemma asserts 
that a holomorphic critical germ $f:\left(\mathbb{C}^{n}, 0\right) \rightarrow(\mathbb{C}, 0)$, whose Hessian determinant is nonzero at the origin, is right equivalent to $x_{1}^{2}+\ldots+x_{n}^{2}$. If one tightens the notion of right equivalence by stipulating that the coordinate change has to be volume-preserving, then one gets the classical theorem by Vey asserting that there is a volume-preserving coordinate transformation mapping $f$ to $\Psi\left(x_{1}^{2}+\ldots+x_{n}^{2}\right)$ where $\Psi \in \mathbb{C}\{t\}$. There is another proof of this result by Garay even of a much more general statement, the isochoric versal unfolding theorem. I have chosen isochoric as a synonym for volume-preserving since as far as I know it is used in technical areas as well (isochoric process). In the third and fourth section we deal with the theory of isochoric unfoldings. We explain how the Brieskorn module and hence $\mu(f)$ enters the picture. To understand the proof of the versality theorem we have given background material in section two. In the fifth section we show how one can prove Vey's lemma using the theory developed so far. Now there is even a third proof of Vey's lemma by Françoise ([Fra78 $)$ whose idea was the following. Assume that you already have the desired relation

$$
f \circ \Phi(\mathbf{x})=\Psi\left(x_{1}^{2}+\ldots+x_{n}^{2}\right) \text { with } \Psi(t)=t+o(t), \text { say } .
$$

Putting $\Psi(t)=t u(t)^{2}$ for some $u$ with $u(0) \neq 0$ one rewrites the relation as

$$
f \circ \Psi(\mathbf{x})=\left[x_{1} u\left(x_{1}^{2}+\ldots+x_{n}^{2}\right)\right]^{2}+\ldots+\left[x_{n} u\left(x_{1}^{2}+\ldots+x_{n}^{2}\right)\right]^{2} .
$$

Then the map

$$
\left(x_{1}, \ldots, x_{n}\right) \mapsto\left(x_{1} u\left(x_{1}^{2}+\ldots+x_{n}^{2}\right), \ldots, x_{n} u\left(x_{1}^{2}+\ldots+x_{n}^{2}\right)\right)
$$

is a coordinate transformation and once it is applied, we can reduce the problem to a problem on the Brieskorn module. In the last section of chapter two we we generalize the approach by Françoise to arbitrary quasihomogeneous polynomials $P$ instead of the $x_{1}^{2}+\ldots+x_{n}^{2}$ in the lemmas 2.19 2.21 and 2.22. They deal with the above-mentioned coordinate change which was only sketched in Françoise's paper. Having established this, we can use a nonisolated version of the Brieskorn module which was already considered in Fra82 to deduce a normal form for $P\left(x_{1}, \ldots, x_{n}\right)=x_{1} \cdots x_{n}$. Uniqueness will be proved by integration over the fibre. The following is the main new result of the whole chapter and it was was recently published in [Sza12a]. 


\section{Introduction}

Theorem. Let $f:\left(\mathbb{C}^{n}, 0\right) \rightarrow(\mathbb{C}, 0)$ be a holomorphic germ that is right equivalent to the product of all coordinates: $f \sim x_{1} \cdots x_{n}$. Then there exists a volumepreserving automorphism $\Phi:\left(\mathbb{C}^{n}, 0\right) \rightarrow\left(\mathbb{C}^{n}, 0\right)$ and an automorphism $\Psi:(\mathbb{C}, 0) \rightarrow(\mathbb{C}, 0)$ such that

$$
f(\Phi(\mathbf{x}))=\Psi\left(x_{1} \cdots x_{n}\right) .
$$

$\Psi$ is uniquely determined by $f$ up to a sign.

One of the classical interpretations of the Milnor number $\mu(f)$ is as the dimension of the parameter space of its semi-universal unfolding. Let $f, g \in$ $\mathbb{C}\{x, y\}$ be vanishing at the origin and coprime. In [Suw83] Suwa has shown that for meromorphic maps $(f: g):\left(\mathbb{C}^{2}, 0\right) \rightarrow \mathbb{P}^{1}$ semi-universal unfoldings exist and the parameter space has dimension $\operatorname{dim}_{\mathbb{C}} \frac{\langle f, g\rangle}{\left\langle f_{x} g-f g_{x}, f g_{y}-g f_{y}\right\rangle}$, once this is finite. Because of that I have come to the conjecture that there must be a relation between this number and other ingredients from the pencil $s f+t g,(s: t) \in \mathbb{P}^{1}$. And indeed there is and this is the content of the third chapter. In the first section we give general results about the geometry of the pencil. In particular we give some equivalent conditions for the bifurcation set $\mathscr{B}(f, g)$, the set corresponding to those pencil members which are special compared to the generic one. In this section we will see that the formula

$$
i\left(f, f_{x} g_{y}-f_{y} g_{x}\right)=\mu(f)+i(f, g)-1
$$

(where $i$ is the intersection number) is essential. Using the Brieskorn module I have found an interesting proof for

$$
\operatorname{gcd}\left(f, f_{x} g_{y}-f_{y} g_{x}\right)=1 \Leftrightarrow f \text { isolated singularity, } \operatorname{gcd}(f, g)=1
$$

which is part of the just mentioned formula.

In the second section I introduce the following numbers

$$
\begin{aligned}
& \mu(f, g)=\operatorname{dim}_{\mathbb{C}} \frac{\mathbb{C}\{x, y\}}{\left\langle f_{x} g-f g_{x}, f_{y} g-f g_{y}\right\rangle}, \\
& v(f, g)=\operatorname{dim}_{\mathbb{C}} \frac{\langle f, g\rangle}{\left\langle f_{x} g-f g_{x}, f_{y} g-f g_{y}\right\rangle} .
\end{aligned}
$$


I consider $\mu(f, g)$ as a generalization of the classical Milnor number of a single function to the Milnor number of a pair of functions. After some elementary properties are given in section two, the first main result is theorem 3.16 .

Theorem. Let $f, g \in \mathbb{C}\{x, y\}$ be nontrivial germs that vanish at the origin. Then the following statements are equivalent:

(i) $\mu(f, g)<\infty$

(ii) $v(f, g)<\infty$

(iii) $\operatorname{gcd}(f, g)=1$ and every linear combination $s f+\operatorname{tg}\left(\right.$ with $\left.(s: t) \in \mathbb{P}^{1}\right)$ has an isolated critical point at the origin.

This relates the a priori infinitely many conditions (iii) to just the single (i). Now $\mathscr{B}(f, g)$ is the finite subset of $\mathbb{P}^{1}$ where the pencil member has higher Milnor number than the generic one. The natural question is whether $\mathscr{B}(f, g)$ can have arbitrary finite cardinality. This is answerered positively in an even stronger form in our second result, proposition 3.24

Theorem. Let $n \geq 0$ be a given integer. Then we can give explicitely $f, g \in$ $\mathfrak{m}_{\mathbb{C}^{2}, 0}$ with $\mu(f, g)<\infty$ such that $\mathscr{B}(f, g)$ has cardinality $n$.

In the fourth section we give an account on the following conjecture 3.25 whose invention I described above.

Conjecture (Bifurcation Formula). Assume that $f, g \in \mathbb{C}\{x, y\}$ both vanish at the origin and that $\mu(f, g)$ is finite. If $\mu_{g e n}$ denotes the generic Milnor number and $\mathscr{B}^{*}=\mathscr{B}(f, g) \backslash\{0, \infty\}$ the reduced bifurcation set of the local pencil generated by $f$ and $g$ (in this order), then the following relation holds:

$$
\mu(f, g)=\mu(f \cdot g)+\sum_{(s: t) \in \mathscr{B}^{*}}\left(\mu(s f+t g)-\mu_{g e n}\right) .
$$

Unfortunately I was not able to prove this conjecture. However I have trust in its validity due to numerous examples which I computed partially by hand or in most cases using the computer algebra system SINGULAR. I have written a SINGULAR library in the appendix with which it is easy to compute all numbers that appear in the bifurcation formula for polynomial input $(f, g)$. 


\section{Introduction}

One should note that my first result says that the left-hand side of the bifurcation formula is finite if and only if the right-hand side is. Finally I have proven the conjecture in a special case and sketched a possible idea for a general proof in the fourth section. We have proposition 3.27

Proposition. The bifurcation formula is true when at least one of $f, g$ is a smooth germ.

The results obtained in chapter three will be published in [Sza12b]. 


\section{Contents}

Introduction vii

1. Geometry of Singularities 1

1.1. Topological and Algebraic Properties of Singularities . . . . . . 1

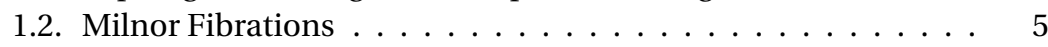

2. Milnor Number and Volume-Preserving Geometry 11

2.1. The Hunting for Isochoric Normal Forms . . . . . . . . . . . . 11

2.2. Brieskorn Modules And Integrals Over Cycles . . . . . . . . . . 15

2.3. Isochoric Versal Unfoldings $\ldots \ldots \ldots \ldots$

2.4. Proof of the Isochoric Versality Theorem . . . . . . . . . . . 22

2.5. Vey's Isochoric Morse Lemma . . . . . . . . . . . . . . . . 27

2.6. An Isochoric Normal Form for Reduced Normal Crossings. . . . 35

3. Milnor Number and Meromorphic Germs 46

3.1. Pencils and Bifurcation . . . . . . . . . . . . . . . 46

3.2. Milnor Number of Pairs of Functions . . . . . . . . . . . . 55

3.3. Singularities in the Pencil . . . . . . . . . . . . . . 65

3.4. The Bifurcation Formula . . . . . . . . . . . . . . . . 70

\begin{tabular}{ll}
\hline A. Appendix & 79
\end{tabular}

A.1. Implementation in SINGULAR . . . . . . . . . . . . . . 79

\begin{tabular}{ll}
\hline Bibliography & 91
\end{tabular} 



\section{Geometry of Singularities}

\subsection{Topological and Algebraic Properties of Singularities}

Most of the material of this section can be found in the books [Dim92, GLS07] and [Mil68].

Following standard conventions we denote by $V(I)$ the vanishing locus of an ideal of functions and by $\sqrt{I}$ the radical ideal of $I$. Since we usually work on germs of functions, $V(I)$ is a set germ. Occasionally we abbreviate the partial derivatives $\partial_{1}, \ldots, \partial_{n}$ simply by a single $\partial$.

Let $\mathbb{C}\left\{x_{1}, \ldots, x_{n}\right\}$ be the power series ring in $n$ complex variables. Sometimes this ring is denoted as $\mathscr{O}_{\mathbb{C}^{n}, 0}$ emphasizing that it is the stalk of the sheaf of germs of holomorphic functions on the $n$-dimensional complex vector space. Its maximal ideal is denoted by $\mathfrak{m}_{\mathbb{C}^{n}, 0}$. An element of $\mathbb{C}\{\mathbf{x}\}$, $f:\left(\mathbb{C}^{n}, \mathbf{0}\right) \rightarrow \mathbb{C}$ is nothing but a holomorphic function germ. If $f \in \mathfrak{m}_{\mathbb{C}^{n}, 0}$, then by $V(f)$ we denote the germ of a set, whereas by $(f=0)$ we will denote the set of zeros of $f$ not as a germ but for a fixed representative of $f$.

We say that $f$ has an isolated singularity at the origin (or is an isolated singularity) if its critical locus $\operatorname{Crit}(f)=\left\{\mathbf{x} \in\left(\mathbb{C}^{n}, \mathbf{0}\right) \mid \partial_{1} f(\mathbf{x})=0, \ldots, \partial_{n} f(\mathbf{x})=\right.$ $0\}$ is either void or the origin itself. The ring $\mathbb{C}\left\{x_{1}, \ldots, x_{n}\right\}$ is factorial and a non-unit $f$ is called reduced if in its prime factor decomposition no multiple factor occurs. We have

Proposition 1.1. Let $f:\left(\mathbb{C}^{n}, \mathbf{0}\right) \rightarrow(\mathbb{C}, 0)$ be a holomorphic germ.

1. If $f$ has an isolated singularity at the origin, then it is reduced.

2. If $n=2$ and $f$ is reduced, then $f$ has an isolated singularity at the origin. 


\section{Geometry of Singularities}

3. On the level of germs of sets we have $V(\partial f) \subset V(f)$.

To two plane germs $f, g:\left(\mathbb{C}^{2}, \mathbf{0}\right) \rightarrow \mathbb{C}$ we can assign their intersection number $i(f, g)$. It can be defined as the dimension of the vector space $\frac{\mathbb{C}\{x, y\}}{\langle f, g\rangle}$. With this definition it follows that $i(f, g)$ is zero if and only if $f(0) \neq 0$ or $g(0) \neq 0$. Furthermore $i(f, g)<\infty$ if and only if $f$ and $g$ have no common factor. If $g$ is irreducible and $\gamma(t)$ is a parametrization of $V(g)$, then $i(f, g)$ can be computed as the order in $t$ of $f \circ \gamma(t)$.

To any holomorphic germ $f:\left(\mathbb{C}^{n}, \mathbf{0}\right) \rightarrow \mathbb{C}$ we can assign the Milnor number $\mu(f)$ which can be given as the dimension of the vector space $\frac{\mathbb{C}\left\{x_{1}, \ldots, x_{n}\right\}}{\left\langle\partial_{1} f, \ldots, \partial_{n} f\right\rangle}$. This number is finite if and only if $f$ has an isolated singularity at the origin.

If $f:\left(\mathbb{C}^{n}, \mathbf{0}\right) \rightarrow(\mathbb{C}, 0)$ is a holomorphic germ, we can take a sufficiently small sphere $S_{\epsilon}^{2 n-1}$ (of radius $0<\epsilon \ll 1$ around the origin $\mathbf{0}$ ) and intersect it with the vanishing locus of $f$. This defines a link $L:=S_{\epsilon}^{2 n-1} \cap(f=0) \hookrightarrow S_{\epsilon}^{2 n-1}$ whose isotopy type is independent of the choice of $\epsilon$. If we remove this exceptional set from the sphere we obtain the classical (knottheoretic) Milnor fibration:

Theorem 1.2 (Milnor fibration).

Let $f:\left(\mathbb{C}^{n}, \mathbf{0}\right) \rightarrow(\mathbb{C}, 0)$ be a holomorphic germ. Then for any sufficiently small $\epsilon>0$, the map

$$
\frac{f}{|f|}: S_{\epsilon}^{2 n-1} \backslash L \rightarrow S^{1}
$$

is a smooth locally trivial fibration.

The (diffeomorphism type of the) fibre of this fibration is called the Milnor fibre of $f$. Since the topology of hypersurface singularities is so closely related with knots resp. links we must dwell a little on that subject. One has the following theorem of Milnor and Burghela-Verena:

Proposition 1.3 (Conic Structure Lemma). Let $f:\left(\mathbb{C}^{n}, \mathbf{0}\right) \rightarrow(\mathbb{C}, 0)$ be a holomorphic germ. Then the vanishing locus of $f$ is homeomorphic to the cone over the link of $f$ with apex at $\mathbf{0}$. This homeomorphism extends to the natural homeomorphism of the closed ball with the cone over the sphere. To be precise, if we denote by ${\overline{B_{\epsilon}}}^{2 n}$ the closed $\epsilon$-ball 


\subsection{Topological and Algebraic Properties of Singularities}

around $\mathbf{0}$, then for sufficiently small $\epsilon>0$ we have a homeomorphism of pairs

$$
\left.{\overline{\left(B_{\epsilon}\right.}}^{2 n},{\overline{B_{\epsilon}}}^{2 n} \cap(f=0)\right) \approx \operatorname{Cone}\left(S_{\epsilon}^{2 n-1}, S_{\epsilon}^{2 n-1} \cap(f=0)\right) .
$$

In particular $V(f)$ is contractible, whereas $V(f) \backslash\{0\}$ is homotopy equivalent to a disjoint union of circles with the same number of components as the link has. Furthermore, the complement ${\overline{B_{\epsilon}}}^{2 n} \backslash(f=0)$ has the same homology as that stated in the next proposition.

Proposition 1.4. Let $L \hookrightarrow S^{3}$ be a link with $m$ components. Then

$$
\begin{aligned}
& H_{0}\left(S^{3} \backslash L ; \mathbb{Z}\right)=\mathbb{Z} \\
& H_{1}\left(S^{3} \backslash L ; \mathbb{Z}\right)=\mathbb{Z}^{m} \\
& H_{2}\left(S^{3} \backslash L ; \mathbb{Z}\right)=\mathbb{Z}^{m-1} \\
& H_{i}\left(S^{3} \backslash L ; \mathbb{Z}\right)=0, i \geq 3 .
\end{aligned}
$$

It is possible to describe explicitely a basis for the first homology of the link complement. On each connected component of the link fix a point and choose a small transversal slice through this point. Then in the transversal slice take a loop encircling the distinguished point. When we do this for all the distinguished points we get a collection of loops which provides a basis for $H_{1}\left(S^{3} \backslash L ; \mathbb{Z}\right)$. The following theorem is valid with modifications in higher dimensions as well, the proof uses however the Gysin sequence, we refer to Dim92.

Theorem 1.5. Let $f=f_{1}^{m_{1}} \ldots f_{r}^{m_{r}}$ be the prime factor decomposition of $f \in$ $\mathfrak{m}_{\mathbb{C}^{2}, 0}$. Then the Milnor fibre $F$ of $f$ has exactly $m:=\operatorname{gcd}\left(m_{1}, \ldots, m_{r}\right)$ connected components and for any base point $z \in F$ we have an exact sequence

$$
0 \rightarrow\left[\pi_{1}\left(S_{\epsilon}^{3} \backslash L, z\right), \pi_{1}\left(S_{\epsilon}^{3} \backslash L, z\right)\right] \rightarrow \pi_{1}(F, z) \rightarrow \mathbb{Z}^{r-1} \rightarrow 0
$$

Proof. The Milnor fibration $F \rightarrow S_{\epsilon}^{3} \backslash L \rightarrow S^{1}$ yields the exact homotopy sequence

$$
0 \rightarrow \pi_{1}(F, z) \rightarrow \pi_{1}\left(S_{\epsilon}^{3} \backslash L, z\right) \rightarrow \pi_{1}\left(S^{1}, f(z)\right) \rightarrow \pi_{0}(F) \rightarrow \pi_{0}\left(S_{\epsilon}^{3} \backslash L\right) \rightarrow \pi_{0}\left(S^{1}\right)=0 .
$$

First recall that the link complement is connected. Therefore by the universal property of the abelianization we can factor one of the involved maps by 


\section{Geometry of Singularities}

the Hurewicz homomorphism $H$

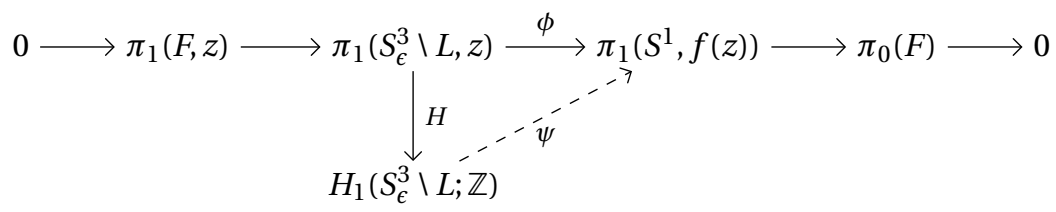

The map $\psi$ sends a small transversal loop $\gamma_{i}$ corresponding to the link component $V\left(f_{i}\right) \cap S$ to its image in $S^{1}$ by composition with $f /|f|$. Choose a sufficiently small neighbourhood of a fixed point on $\left(f_{i}=0\right) \cap S_{\epsilon}^{3}$ so that when we write

$$
\frac{f}{|f|}=\frac{f_{i}^{m_{i}}}{\left|f_{i}^{m_{i}}\right|} \prod_{j=1, j \neq i}^{r} \frac{f_{j}^{m_{j}}}{\left|f_{j}^{m_{j}}\right|}
$$

the product term is nonzero and such that the point is given in local coordinates by $(x, y)=(0,0)$, the function is $f_{i}=x$ and the transversal loop is $\gamma(t)=\left(\delta e^{i 2 \pi t}, 0\right)$ where $t \in[0,1]$ and $\delta<<1$. Then after a coordinate transformation we have

$$
\frac{f}{|f|} \circ \gamma(t)=\frac{x^{m_{i}}}{\left|x^{m_{i}}\right|} \circ \gamma(t)=e^{i 2 \pi m_{i} t}
$$

Hence $\Psi$ maps the basis $\gamma_{1}, \ldots, \gamma_{r}$ of $H_{1}\left(S_{\epsilon}^{3} \backslash L ; \mathbb{Z}\right)$ to $m_{1}\left[S^{1}\right], \ldots, m_{r}\left[S^{1}\right] \bmod$ ulo orientation. The image of $\Psi$ is therefore $m \mathbb{Z}$ where $m=\operatorname{gcd}\left(m_{1}, \ldots, m_{r}\right)$ and since $H$ is surjective we get $\pi_{0}(F) \cong \mathbb{Z} / m \mathbb{Z}$, hence the Milnor fibre has $m$ connected components. The final assertion about the exact sequence is now easily seen.

Corollary 1.6. A reduced and a nonreduced holomorphic germ cannot be right equivalent under a homeomorphism.

The following remarkable theorem is due to various people. It states that a certain module is free and of finite rank. In 1970, E. Brieskorn and P. Deligne proved the finiteness of the module and M. Sebastiani proved its torsionfreeness. A nice proof of the latter has also been given by B. Malgrange.

Theorem 1.7. ([Bri70], [Seb70], [Mal74])

If $f:\left(\mathbb{C}^{n}, \mathbf{0}\right) \rightarrow(\mathbb{C}, 0)$ has an isolated singularity at the origin, then the Brieskorn 
module

$$
H_{f}^{\prime \prime}=\frac{\Omega^{n}}{d f \wedge d \Omega^{n-2}}
$$

is a free $\mathbb{C}\{f\}$-module of $\operatorname{rank} \mu(f)$.

In the second chapter we will examine this and related modules more closely.

\subsection{Milnor Fibrations}

In this section we introduce the Milnor-Lê fibrations not only for a single holomorphic function germ $f:\left(\mathbb{C}^{m}, 0\right) \rightarrow(\mathbb{C}, 0)$ but for a map germ $f:\left(\mathbb{C}^{m}, 0\right) \rightarrow$ $\left(\mathbb{C}^{k}, 0\right)$ which defines an isolated complete intersection singularity. We indicate how the fibration theorem can be proved. On the one hand we will need this more complicated situation for the generalization of the Brieskorn module to a parametrized version due to Greuel in theorem 2.3. On the other hand, a more thorough discussion of the fibration theorem is neccessary in order to understand the difficulties for nonisolated singularities, which will be require a different strategy. The function $f\left(x_{1}, \ldots, x_{n}\right)=x_{1} \cdots x_{n}$ gives a nonisolated singularity to which the fibration theorem is applied in the last section of chapter two. For the isolated complete intersection case we refer to [Lo084. For the nonisolated case the reader is referred to the paper [LT83.

Fix some natural numbers $m>k$ and let $f:\left(\mathbb{C}^{m}, p\right) \rightarrow\left(\mathbb{C}^{k}, 0\right)$ be a holomorphic germ. We denote by $\operatorname{Jac}(f)$ the ideal in $\mathscr{O}_{\mathbb{C}^{m}, p}$ which is generated by the determinants of the $k$-minors of the Jacobian matrix of $f$. Let $\operatorname{Crit}(f)$ be the critical locus of $f$. This is the (germ of an) analytic set which is defined by the vanishing of the ideal $\operatorname{Jac}(f)$.

Proposition 1.8. Let $f:\left(\mathbb{C}^{m}, p\right) \rightarrow\left(\mathbb{C}^{k}, 0\right)$ be as above. Denote by $X_{0}$ a sufficiently small representant of the complex space germ $\left(f^{-1}(0), p\right)$. Then the following statements are equivalent:

1. $f$ is flat at $p$ and $p$ is an isolated singular point of $X_{0}$.

2. $\operatorname{dim}\left(X_{0}, p\right)=m-k$ and $p$ is an isolated singular point of $X_{0}$. 


\section{Geometry of Singularities}

3. $\operatorname{dim}\left(X_{0} \cap \operatorname{Crit}(f), p\right) \leq 0$, i.e. $X_{0} \cap \operatorname{Crit}(f)$ is either empty or equals $\{p\}$.

4. $\mathscr{O}_{\mathbb{C}^{m}, p} / \operatorname{Jac}(f)$ is a finite $\mathscr{O}_{\mathbb{C}^{k}, 0}$-module (with module structure induced by $f$ ).

5. The restriction $f_{\mid}:(\operatorname{Crit}(f), p) \rightarrow\left(\mathbb{C}^{k}, 0\right)$ is a finite map germ.

The use of the scheme-theoretic fibre is important as one can see from the example $f(x, y)=x^{2}$. The above proposition was mentioned without proof in Gre75 for the more general case when $\left(\mathbb{C}^{m}, p\right)$ is replaced by the germ of a complete intersection of the same dimension. (A proof can however be found in Greuel's thesis.)

In the situation of the last proposition, one says that $f=\left(f_{1}, \ldots, f_{k}\right)$ defines an isolated complete intersection singularity $\left(X_{0}, p\right)$ at $p$. In this case the ideal $\left\langle f_{1}, \ldots, f_{k}\right\rangle$ is radical in the ring $\mathscr{O}_{\mathbb{C}^{m}, p}$ ([Loo84], prop. 1.10).

For real numbers $\epsilon, \delta>0$ denote by $B_{\epsilon}(p)$ resp. $\bar{B}_{\epsilon}(p)$ the open resp. closed $\epsilon$-ball around $p \in \mathbb{C}^{m}$ with boundary $S_{\epsilon}(p)$ and by $D_{\delta}(0)$ the open $\delta$-ball around the origin 0 in $\mathbb{C}^{k}$. Let $D(f):=f(\operatorname{Crit}(f))$ be the discriminant of $f$ as a set (germ). Following Looijenga ([Loo84],p. 25f.) we introduce the following standard notations:

$$
\begin{aligned}
S & =D_{\delta}(0) \\
\bar{X} & =\bar{B}_{\epsilon}(p) \cap f^{-1}(S) \\
X & =B_{\epsilon}(p) \cap f^{-1}(S) \\
S^{\prime} & =S \backslash D(f) \\
X^{\prime} & =B_{\epsilon}(p) \cap f^{-1}\left(S^{\prime}\right) \\
\bar{X}^{\prime} & =\bar{B}_{\epsilon}(p) \cap f^{-1}\left(S^{\prime}\right) \\
\partial X^{\prime} & =\partial B_{\epsilon}(p) \cap f^{-1}\left(S^{\prime}\right) .
\end{aligned}
$$

By construction, $f: X^{\prime} \rightarrow S^{\prime}$ is submersion. Even more holds.

Theorem 1.9 (([|Ham71], $\mid \overline{\text { Mil68 }])) . ~(M i l n o r-L e ̂ ~ f i b r a t i o n) ~}$ If $f:\left(\mathbb{C}^{m}, p\right) \rightarrow\left(\mathbb{C}^{k}, 0\right)$ defines an isolated complete intersection singularity, 
then for all sufficiently small $\epsilon>0$ and sufficiently small $0<\delta \ll \epsilon$, the map

$$
f:\left(\bar{X}^{\prime}, \partial X^{\prime}\right) \rightarrow S^{\prime}
$$

is a smooth $\left(=C^{\infty}\right)$ locally trivial fibre bundle pair.

The map is written in such a way that $\epsilon$ and $\delta$ do not appear anymore. This is standard practise not only due to convenience. In fact, for another choice of $1 \gg \epsilon \gg \delta>0$ we obtain a diffeomorphic fibration ([Loo84],prop. 2.9).

In general of course we cannot expect the discriminant to be just the origin. In fact, for an isolated complete intersection singularity the discriminant is an analytic set in $\left(\mathbb{C}^{k}, 0\right)$ of the same dimension at 0 as $\operatorname{dim}(\operatorname{Crit}(f), p)$. This follows from the finite coherence theorem and the fact that the dimension does not change under the finite map $f_{\mid}:(\operatorname{Crit}(f), p) \rightarrow\left(\mathbb{C}^{k}, 0\right)$. For example for an isolated hypersurface singularity $f:\left(\mathbb{C}^{m}, p\right) \rightarrow(\mathbb{C}, 0)$ we have by definition $\operatorname{dim}(\operatorname{Crit}(f), p) \leq 0$ and hence the discriminant is an analytic set germ of dimension $\leq 0$ in $(\mathbb{C}, 0)$. In other words it consists of at most one point. This is included in proposition 1.1 part (3) as well. What follows is the classical

Theorem 1.10. (Milnor's fibration theorem) Let $f:\left(\mathbb{C}^{m}, p\right) \rightarrow(\mathbb{C}, 0)$ be a holomorphic germ with $\operatorname{Crit}(f) \subset\{p\}$. Then for all sufficiently small $0<\delta \ll \epsilon \ll 1$, the map

$$
f:\left(\bar{B}_{\epsilon}(p), S_{\epsilon}(p)\right) \cap f^{-1}\left(D_{\delta}(0) \backslash\{0\}\right) \rightarrow D_{\delta}(0) \backslash\{0\}
$$

is a smooth locally trivial fibre bundle pair.

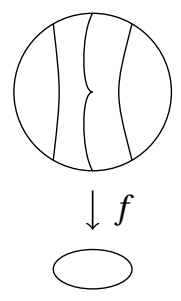

The induced fibration $f: X^{\prime} \rightarrow S^{\prime}$ is, when restricted on some small circle $S_{\delta}^{1} \subset S^{\prime}$, equivalent to the knottheoretic Milnor fibration in theorem 1.2 (i.e. 


\section{Geometry of Singularities}

there is a diffeomorphism of circles and of the total spaces of the fibrations which makes a commutative diagram). The general fibre is also called the Milnor fibre $\mathrm{Mil}_{f, 0}$ (and this is the one we usually deal with) and its only nonvanshing homology groups are $H_{0}\left(\operatorname{Mil}_{f, 0} ; \mathbb{Z}\right)=\mathbb{Z}$ and $H_{n-1}\left(\operatorname{Mil}_{f, 0}\right)=\mathbb{Z}^{\mu(f, 0)}$. In fact, Milnor has shown in Mil68] that the Milnor fibre of $f:\left(\mathbb{C}^{n}, 0\right) \rightarrow(\mathbb{C}, 0)$ with an isolated critical point at the origin is homotopy equivalent to a $\mathrm{CW}$ complex of dimension $n-1$ (which is remarkable since the Milnor fibre is a complex manifold of complex dimension $n-1)$ and its $(n-1)$ th homology group has rank equal to the degree of the map $\partial f /\|\partial f\|: S_{\epsilon}^{2 n-1} \rightarrow S^{2 n-1}$. Palamodov has shown that this is equal to the formula already given in the first section $\mu(f, 0)=\operatorname{dim}_{\mathbb{C}} \mathbb{C}\{\mathbf{x}\} /\left\langle\partial_{1} f, \ldots, \partial_{n} f\right\rangle$.

As was noticed by Lê and others, for a general map germ $f:\left(\mathbb{C}^{m}, p\right) \rightarrow$ $\left(\mathbb{C}^{k}, 0\right)$, i.e. not neccessarily an isolated complete intersection singularity, there need not exist a fibration in a neighbourhood of $p$ and away from the discriminant locus. I will refer to such a fibration as of Milnor-Lê type.

Consider for the following standard example

$$
f:\left(\mathbb{C}^{3}, 0\right) \rightarrow\left(\mathbb{C}^{2}, 0\right), f(x, y, z)=\left(x^{2}-y^{2} z, y\right) .
$$

This map germ is flat and all fibres (even the special fibre) are smooth and $f$ is a submersion away from the zero fibre. However, the critical locus of $f$ is not isolated in $f^{-1}(0)$, but comprises the whole fibre. This makes it impossible to apply the fibration theorem. Since moreover some of its nonspecial fibres are connected, some are not (check $f^{-1}(0, \delta)$ and $f^{-1}\left(\delta^{2}, 0\right)$ ), a fibration of Milnor-Lê type does not exist for this example.

Let us now sketch a proof of the fibration theorem. The main ingredient is the Ehresmann lemma which we recall. Let $E$ be a smooth manifold with boundary and $B$ be a smooth manifold, connected for simplicity. Let $f: E \rightarrow B$ be a proper smooth map such that $\operatorname{rk}(f)=\operatorname{dim} B$ and $\operatorname{rk}(f \mid \partial E)=$ $\operatorname{dim} B$ everywhere. Then $f:(E, \partial E) \rightarrow B$ is a smooth locally trivial fibre bundle pair. This theorem will be applied to $f_{\mid}: \bar{B}_{\epsilon}(p) \cap f^{-1}\left(D_{\delta}(0) \backslash D(f)\right) \rightarrow$ $D_{\delta}(0) \backslash D(f)$ which is clearly proper since $\bar{B}_{\epsilon}(p)$ is compact. The condition $\operatorname{rk}(f)=\operatorname{dim} B$ is of course fulfilled since we have already taken away the discriminant locus where $f$ is not a submersion. The second rank con- 
dition $\operatorname{rk}(f \mid \partial E)=\operatorname{dim} B$ is the troublemaker. It is a priori not clear whether $\partial E=S_{\epsilon}(x) \cap f^{-1}\left(D_{\delta}(0) \backslash D(f)\right)$ is a manifold at all. The basic idea is that - since the fibre $f^{-1}(0)$ has $x$ as an isolated singular point, the space $S_{\epsilon}(x) \cap f^{-1}(0)$ will be smooth conjecturally. In fact, one can use the curve selection lemma by Milnor to show that this intersection is transverse for all small $\epsilon$.

Apart from the proof of the Milnor-Lê fibration theorem with the Ehresmann lemma one could also use the Thom isotopy lemma. Of course, then we would not get a smooth fibration, but still a topological one which suffices for most purposes. A basic condition behind behind the Thom lemma is the so called Thom $a_{f}$-condition, a relative Whitney-(a) condition. But this condition can also be used in the smooth context. According to Hironaka ([Hir77]) for a holomorphic function germ $f \in \mathfrak{m}_{\mathbb{C}^{n}, 0}$ one can find a representative which has a complex-analytic Thom-Whitney stratification such that $f^{-1}(0)$ is a union of strata. We show how one can conclude the fibration theorem using this. It is a known property of Whitney stratifications ("Bertini-Sard") that by taking $\epsilon$ smaller if necessary, $S_{\epsilon}(0)$ intersects all strata transversely, in particular those of $f^{-1}(0)$. The image space has as stratification the one consisting of $\{0\}$ and its open complement (recall that all critical points of $f$ are known to be in $\left.f^{-1}(0)\right)$. Since the $a_{f}$-condition is satisfied for these two stratifications, we can show that every $f^{-1}(t), t \in D_{\delta}(f(0)) \backslash f(0)$ intersects $S_{\epsilon}$ transversely, for any sufficiently small $\delta<<\epsilon$ (transverse intersection property). We argue by contradiction. So if this was not the case there would exist a sequence $y_{i} \in S_{\epsilon}$ converging to some $x \in f^{-1}(f(0)) \cap S_{\epsilon}$ such that $T_{y_{i}} f^{-1}\left(f\left(y_{i}\right)\right)$ is not transversal to $S_{\epsilon}$ at $y_{i}$. Since the real codimension of $S_{\epsilon}$ is one, this is equivalent to say $T_{y_{i}} f^{-1}\left(f\left(y_{i}\right)\right) \subset T_{y_{i}} S_{\epsilon}$. By compactness of the Grassmannian we can take a subsequence such that the left hand side converges (for the right it is clear): $\lim _{i} T_{y_{i}} f^{-1}\left(f\left(y_{i}\right)\right) \subset \lim _{i} T_{y_{i}} S_{\epsilon}=T_{x} S_{\epsilon}$. If we denote by $S$ the stratum of $f^{-1}(f(0))$ which contains $x$, we have by Thom$a_{f}$ that $T_{x} S \subset \lim _{i} T_{y_{i}} f^{-1}\left(f\left(y_{i}\right)\right)$. Taking together this implies $T_{x} S \subset T_{x} S_{\epsilon}$. Hence $f^{-1}(0)$ is not stratified-transverse to $S_{\epsilon}$, a contradiction. This transverse intersection property suffices to get the fibration by the use of the Ehresmann lemma again. More general one even has the following theorem by Lê ([Lê77]) where $X$ is arbitary analytic.

Theorem 1.11. (Lê's fibration theorem) Let $X \subset U \subset \mathbb{C}^{n}$ be an analytic subset of an open subset $U$ in $\mathbb{C}^{n}$. Let $f: X \rightarrow \mathbb{C}$ 


\section{Geometry of Singularities}

be an analytic function with $f(x)=0$ for some $x \in X$. Then for $0<\delta \ll \epsilon \ll 1$, the restriction of $f$,

$$
f: B_{\epsilon}(0) \cap X \cap f^{-1}\left(D_{\delta}^{*}\right) \rightarrow D_{\delta}^{*}
$$

is a stratified locally trivial fibration.

We have seen already a couple of different definitions of the Milnor number $\mu$ of an isolated singularity $f:\left(\mathbb{C}^{n}, 0\right) \rightarrow(\mathbb{C}, 0)$. It is the rank of the $(n-1)$ th homology group of the fibre of any of the Milnor fibrations (i.e. knottheoretic or complex-analytic). It can be computed as a vector space dimension. It the degree of the map $\nabla f /\|\nabla f\|: S_{\epsilon}^{2 n-1} \rightarrow S^{2 n-1}$ where $\nabla=\left(\partial_{1}, \ldots, \partial_{n}\right)$. But it is also the degree of the branched covering $\nabla f:\left(\mathbb{C}^{n}, 0\right) \rightarrow\left(\mathbb{C}^{n}, 0\right)$. In two dimensions $\mu(f)$ is the Hopf invariant of $\left(f_{x}: f_{y}\right): S_{\epsilon}^{3} \rightarrow \mathbb{P}^{1} \cong S^{2}$ since the Hopf invariant of $(x: y): S^{3} \rightarrow \mathbb{P}^{1}$ is equal to one and we have a commutative diagram with $\left(f_{x}, f_{y}\right) /\left\|\left(f_{x}, f_{y}\right)\right\|: S_{\epsilon}^{3} \rightarrow S^{3}$ whose degree is $\mu(f)$. A quite remarkable property was already found by Milnor in his book: $\mu(f)$ equals the number of critical points in a generic (Morse) perturbation $f_{t}$ of $f$. We suggest the reader to have a look at the nice paper by Orlik [Orl77], the classical books by Arnold, Varchenko and Gusein-Zade ( the newer book [Ebe01] by Ebeling. 


\section{Milnor Number and Volume-Preserving Geometry}

\subsection{The Hunting for Isochoric Normal Forms}

For two smooth germs $f$, $g \in \mathfrak{m}_{\mathbb{C}^{n}, 0}$ is is clear by the implicit function theorem that they are right equivalent. It is also true that they are even right equivalent under a volume-preserving automorphism. If $n=2$ then a volume form is nothing but a symplectic form and the just mentioned normalization result is a sharpification of the classical Darboux theorem ([BBT03], p. 520). In this chapter we are concerned with volume-preserving right equivalence of holomorphic function germs. Let us understand why this has something to do with the Milnor number. In the above example of a smooth germ the Milnor number is zero. There is no obstruction to volumepreserving right equivalence once you have usual right equivalence. So let's go one step further. If the Milnor number of some $f \in \mathfrak{m}_{\mathbb{C}^{n}, 0}$ is equal to one, we have a critical Morse germ. The classical holomorphic Morse lemma tells us that $f$ is right equivalent to $x_{1}^{2}+\ldots+x_{n}^{2}$. For the volume-preserving right equivalence we have the following analogous result due to Vey.

Theorem (Volume-Preserving Morse Lemma).

Let $f:\left(\mathbb{C}^{n}, 0\right) \rightarrow(\mathbb{C}, 0)$ be a critical Morse singularity. Then there exists a volumepreserving automorphism $\Phi \in \operatorname{Aut}\left(\mathbb{C}^{n}, 0\right)$ and a holomorphic germ $\Psi:(\mathbb{C}, 0) \rightarrow$ $(\mathbb{C}, 0)$ such that

$$
f \circ \Phi=\Psi \circ Q_{f} .
$$

Here $Q_{f}$ denotes the quadratic part of $f$ at the origin. It is possible to demand $\Psi(t)=t+O\left(t^{2}\right)$ and with this requirement $\Psi$ is uniquely determined by $f$.

So when $\mu=1$ there appears one continuous obstruction for volume-presering right equivalence. This is the function $\Psi$. Nowadays there are three proofs of this result, by Vey, Garay and Françoise. We will give a proof of this theorem using the more general result by Garay, namely the isochoric versal 


\section{Milnor Number and Volume-Preserving Geometry}

unfolding theorem whose proof requires some preparations about isochoric unfoldings and it requires a generalization of the classical Brieskorn module of a hypersurface singularity to an isolated complete intersection singularity by Greuel. The isochoric versality theorem 2.9 can be formulated from a "higher" point of view as follows.

Theorem 2.1. For an isolated singularity $f \in \mathfrak{m}_{\mathbb{C}^{n}, 0}$ there occur $\mu(f, 0)$ continuous moduli for isochoric right equivalence.

We can make this precise in an alternative way, too. Namely, Françoise has described something like the "moduli space" of isochoric right equivalence. Let $f:\left(\mathbb{C}^{n}, 0\right) \rightarrow(\mathbb{C}, 0)$ be an isolated singularity and for right equivalence look only at coordinate transformations whose tangent map at the origin in $\mathbb{C}^{n}$ is the identity. Call this space Aut $\left.\mathbb{C}^{n}, 0\right)$. Let Aut ${ }^{\Omega}\left(\mathbb{C}^{n}, 0\right)$ be the volumepreserving automorphisms and $\operatorname{Aut}_{1}^{\Omega}\left(\mathbb{C}^{n}, 0\right)=\operatorname{Aut}_{1}\left(\mathbb{C}^{n}, 0\right) \cap \operatorname{Aut}^{\Omega}\left(\mathbb{C}^{n}, 0\right)$. Then we define

$$
\mathscr{M}(f):=\frac{\left\{\Phi^{*} f \mid \quad \Phi \in \operatorname{Aut}_{1}\left(\mathbb{C}^{n}, 0\right)\right\}}{\left\{\Phi^{*} f \mid \quad \Phi \in \operatorname{Aut}_{1}^{\Omega}\left(\mathbb{C}^{n}, 0\right)\right\}} .
$$

Fix a basis $\Omega_{1}, \ldots, \Omega_{\mu}$ of the $\mathbb{C}\{t\}$-module $H_{f}^{\prime \prime}$. Then one can define a map of sets

$$
\mathscr{M}(f) \rightarrow \mathbb{C}\{t\}^{\mu}
$$

as follows. Take an element $[g]$ of the left-hand side. Take a representive $g_{1} \epsilon$ [ $g$ ]. By definition of $\mathscr{M}(f)$ there exists $\Phi_{1} \in$ Aut $_{1}\left(\mathbb{C}^{n}, 0\right)$ such that $\Phi_{1}^{*} g_{1}=f$. We can write

$$
\Phi_{1}^{*} \Omega=a_{1} \circ f \Omega_{1}+\ldots+a_{\mu} \circ f \Omega_{\mu}+d \eta
$$

with $d f \wedge d \eta=0$ and uniquely determined (by $\Phi_{1}^{*} \Omega$ ) germs $a_{i} \in \mathbb{C}\{t\}$.

The assignment in equation 2.1 is

$$
[g] \mapsto\left(a_{1}(t), \ldots, a_{\mu}(t)\right) .
$$

This map is well-defined, injective and surjective onto a subspace obtained by killing from $\mathbb{C}\{t\}^{\mu}$ a one-dimensional vector subspace (i.e. the image is isomorphic to the vector space $\mathbb{C}\{t\}^{\mu} / \mathbb{C}$ ).

What is the geometric explanation behind all this? Stokes theorem in the real two-dimensional plane asserts that $\oint_{C} x d y$ computes the area of the in- 
terior that is surrounded by the simple closed curve $C$. When we think of $x, y$ as complex variables and the curve to be a cycle lying in some smooth fibre of a function $f \in \mathfrak{m}_{\mathbb{C}^{2}, 0}$, then one is led to believe that such an integral should be significant for the study of volume-preserving equivalence and for isochoric normal forms in particular. How many cycles (classes) are there in the Milnor fibre? Of course $\mu(f)=\operatorname{rk} H_{n-1}\left(\operatorname{Mil}_{f, 0} ; \mathbb{Z}\right)$ many. The rank of the Brieskorn module is also $\mu(f)$. That this coincidence is not just accidental will become clear in the section about the isochoric versal unfoldings where it will be described why the Brieskorn module enters the game.

Let us investigate the condition that $H_{n-1}\left(\mathrm{Mil}_{f, 0} ; \mathbb{Z}\right)$ has rank equal to one, for this might lead to a single continous obstruction and hence to a normal form if one believes the philosophy. Well for an isolated singularity $f \in \mathfrak{m}_{\mathbb{C}^{n}, 0}$ we know it is equivalent to $\mu(f, 0)=1$ and this is equivalent to $f$ being critical and Morse at the origin. Here we have the lemma of Vey which completely answers the normal form problem. What about nonisolated singularities? Can we list all nonisolated singularities $f \in \mathfrak{m}_{\mathbb{C}^{n}, 0}$ with $\mathrm{rk} H_{n-1}\left(\mathrm{Mil}_{f, 0} ; \mathbb{Z}\right)=1$ ? If so, then each of them may be a potential candidate for an isochoric normal form result. Let us look at dimension two. The following proposition should be well-known, but a proof is difficult to locate.

Proposition 2.2. For $f:\left(\mathbb{C}^{2}, 0\right) \rightarrow(\mathbb{C}, 0)$ the group $H_{1}\left(\mathrm{Mil}_{f, 0} ; \mathbb{Z}\right)$ has rank one if and only if $f$ is right equivalent to $x^{a} y^{b}$ with $\operatorname{gcd}(a, b)=1$.

Proof. Let $H_{1}\left(\operatorname{Mil}_{f, 0} ; \mathbb{Z}\right)$ have rank one. If $f=f_{1}^{q_{1}} \cdots f_{r}^{q_{r}}$ is the decomposition of $f$ into irreducible factors then according to Melle-Hernández ([MH99]) the Euler charateristic of the Milnor fibre of $f$ can be computed from

$$
\chi\left(\operatorname{Mil}_{f, 0}\right)=-\sum_{1 \leq i<j \leq r} i\left(f_{i}, f_{j}\right)\left(q_{i}+q_{j}\right)+\sum_{i=1}^{r} q_{i}\left(1-\mu\left(f_{i}, 0\right)\right) .
$$

From the first chapter we know that the number of connected components $d$ of the Milnor fibre equals the greatest common divisor of the $q_{1}, \ldots, q_{r}$. Since we can write $f-\delta^{d}=\prod_{\xi}\left[\left(f_{1}^{q_{1} / d} \cdots f_{r}^{q_{r} / d}\right)-\xi \delta\right]$ (product over all $d$ th roots of unity, $\delta \in(\mathbb{C}, 0)$ sufficiently small), these components are all diffeomorphic and so we deduce from rk $H_{1}\left(\operatorname{Mil}_{f, 0} ; \mathbb{Z}\right)=1$ that $d=1$. Then $\chi\left(\operatorname{Mil}_{f, 0}\right)=$ $1-1=0$ and we consider the above equation. If $r$ was at least three, then $\sum_{1 \leq i<j \leq r} i\left(f_{i}, f_{j}\right)\left(q_{i}+q_{j}\right) \geq \sum_{i=1}^{r-1} i\left(f_{i}, f_{i+1}\right)\left(q_{i}+q_{i+1}\right)+i\left(f_{1}, f_{r}\right)\left(q_{1}+q_{r}\right)$ is strictly 


\section{Milnor Number and Volume-Preserving Geometry}

larger than $q_{1}+\ldots q_{r-1}+q_{r} \geq \sum_{i=1}^{r} q_{i}\left(1-\mu\left(f_{i}, 0\right)\right)$. So we must have $r=1$ or $r=2$. If $r=1$ (and $d=1$ ) then $f$ is irreducible and hence an isolated singularity. It is well-known that $\mu(f, 0)=1$ implies that $f$ is right equivalent to $x y$. In the remaining case $r=2$ we have the equality

$$
i\left(f_{1}, f_{2}\right)\left(q_{1}+q_{2}\right)=q_{1}\left(1-\mu\left(f_{1}, 0\right)\right)+q_{2}\left(1-\mu\left(f_{2}\right)\right),
$$

so, since $q_{i} \geq 1, i\left(f_{1}, f_{2}\right) \geq 1,1-\mu\left(f_{i}, 0\right) \leq 1$, it follows that $i\left(f_{1}, f_{2}\right)=1$ and $\mu\left(f_{1}, 0\right)=\mu\left(f_{2}, 0\right)=0$, i.e. $f_{1}, f_{2}$ are smooth and transverse. By the inverse function theorem there is an automorphism of $\left(\mathbb{C}^{2}, 0\right)$ which transforms the pair $\left(f_{1}, f_{2}\right)$ to $(x, y)$. Hence $f$ is right equivalent to $x^{q_{1}} y^{q_{2}}$ with coprime $q_{1}, q_{2}$. A different proof could be obtained by using the exact sequence from theorem 1.5 One compares the exact sequences of $f$ to that of the reduced germ $f_{\text {red }}$ and uses results from group theory to conclude that $f_{r e f}$ is Morse.

What about the integral of $\lambda=x d y$ over a generator of $H_{1}\left(\mathrm{Mil}_{f, 0}\right)$ ? Choose real numbers $0<s \ll \epsilon \ll 1$ such that $\operatorname{Mil}_{f}(s)=\left\{(x, y) \in B_{\epsilon}(0) \mid x^{a} y^{b}=s\right\}$ is the Milnor fibre of $f(x, y)=x^{a} y^{b}$ where $a, b \in \mathbb{N}$ are coprime integers. Then we can embed $S^{1}$ into the Milnor fibre over $s$ using the map

$$
S^{1} \ni z \mapsto(x(t), y(t))=\left(z^{b} s^{1 / a+b}, z^{-a} s^{1 / a+b}\right) .
$$

In fact this map is an injective immersion of a compact space, hence an embedding. (The injectivity follows from $\operatorname{gcd}(a, b)=1$.) We now integrate the form $x^{m} y^{n} d y$ along this cycle:

$$
\begin{aligned}
\int_{S^{1}} x^{m} y^{n} d y & =\int_{S^{1}} z^{m b} s^{m /(a+b)} z^{-a n} s^{n /(a+b)}(-a) z^{-a-1} s^{1 /(a+b)} d z \\
& =-a s^{(m+n+1) /(a+b)} \int_{S^{1}} z^{m b-a n-a-1} d z .
\end{aligned}
$$

This integral is nonzero if and only if $m b-a n-a=0$. Of course there are choices of $m, n$ where this is achieved. Thus, the embedded circle is homologically nontrivial, i.e. represents a generator of $H_{1}\left(\mathrm{Mil}_{f, 0} ; \mathbb{C}\right) \cong \mathbb{C}$. Now we let $m=1$ and $n=0$, so that $\lambda=x d y$ is a primitive of the volume form. Its integral is nonzero if and only if $b-a=0$. But since $\operatorname{gcd}(a, b)=1$ this holds if only if $a=b=1$. 
For $a=b=1$ we have Vey's lemma as an isochoric normal form for functions which are right equivalent to $x^{a} y^{b}=x y \sim x^{2}+y^{2}$. But for other coprime $a, b$ an isochoric normal form for functions which are right equivalent to $x^{a} y^{b}$ is unlikely to exist in the simple form $f \circ \Phi=\Psi\left(x^{a} y^{b}\right)$ with $\Phi$ volumepreserving. At least I believe that it might exist in a more complicated form.

Now let us raise dimension. Françoise has considered a nonisolated version of the Brieskorn module and has given explicitely $\operatorname{gcd}\left(m_{1}, \ldots, m_{n}\right)$ generators when $f=x_{1}^{m_{1}} \cdots x_{n}^{m_{n}}$. So one might think that when the exponents are coprime we could look for a normal form. By some technical reason it works under the restriction when all exponents are equal to one. The author has used the techniques from Françoise's proof of Vey's lemma in [Fra78 to generalize its main components to quasihomogeneous polynomials and then it allowed a proof of the following isochoric normal form in higher dimensions.

Theorem. Consider a holomorphic germ $f:\left(\mathbb{C}^{n}, 0\right) \rightarrow(\mathbb{C}, 0)$ that is right equivalent to the product of all coordinates: $f \sim x_{1} \cdots x_{n}$. Then there exists a volumepreserving automorphism $\Phi:\left(\mathbb{C}^{n}, 0\right) \rightarrow\left(\mathbb{C}^{n}, 0\right)$ and an automorphism $\Psi:(\mathbb{C}, 0) \rightarrow$ $(\mathbb{C}, 0)$ such that

$$
f(\Phi(\mathbf{x}))=\Psi\left(x_{1} \cdots x_{n}\right) .
$$

$\Psi$ is uniquely determined by $f$ up to a sign.

\subsection{Brieskorn Modules And Integrals Over Cycles}

In the seminal paper [Bri70] Brieskorn has introduced different $\mathbb{C}\{t\}$-modules for the investigation of the monodromy of an isolated singularity. One of these modules is given for an isolated singularity $f:\left(\mathbb{C}^{n}, 0\right) \rightarrow(\mathbb{C}, 0)$ by

$$
H_{f}^{\prime \prime}=\frac{\Omega_{\mathbb{C}^{n}, 0}^{n}}{d f \wedge d \Omega_{\mathbb{C}^{n}, 0}^{n-2}} .
$$

Here $\Omega_{\mathbb{C}^{n}, 0}^{k}$ denotes the vector space of germs of holomorphic $k$-forms at the origin in $\mathbb{C}^{n}$. The $\mathbb{C}\{t\}$-module structure of this module comes from multiplication with $f$. It is shown in the cited paper together with Sebastiani's 


\section{Milnor Number and Volume-Preserving Geometry}

paper [Seb70], see also Malgrange [Mal74], that this is a free module with rank equal to the Milnor number $\mu(f, 0)$ of $f$ at the origin.

This classical Brieskorn module was extended to isolated complete intersection singularities by Greuel in ([Gre75]). We assume that conditions from proposition 1.8 are fulfilled. I.e. $f:\left(\mathbb{C}^{m}, 0\right) \rightarrow\left(\mathbb{C}^{k}, 0\right)$ defines an isolated complete intersection. The restriction to the Milnor ball, $f: X^{\prime} \rightarrow S^{\prime}$ is a fibre bundle after theorem 1.9 . Its fibre has the homotopy type of a wedge of spheres of dimension $m-k$. Their number is defined to be the Milnor number of $f, \mu(f)$. Let $\Omega_{f}^{s}$ be the stalk at the origin in $\mathbb{C}^{m}$ of the relative holomorphic $s$-forms on $X$ (relative with respect to $f: X \rightarrow S$ ). I.e. we have

$$
\Omega_{f}^{s}=\frac{\Omega_{\mathbb{C}^{m}, 0}^{s}}{\sum_{i=1}^{k} d f_{i} \wedge \Omega_{\mathbb{C}^{m}, 0}^{s-1}} .
$$

Then Greuel defines the $\mathscr{O}_{S, 0}$-module

$$
H_{f}^{\prime \prime}=\frac{\Omega_{\mathbb{C}^{m}, 0}^{m}}{d f\left(d \Omega_{f}^{n-1}\right)} .
$$

Theorem 2.3 (Gre75, prop. 4.8). With the above assumptions and notations, $H_{f}^{\prime \prime}$ is free of rank $\mu(f)$.

One can ask if it possible to gain similar results for nonisolated singularities. Following analogy we face the problem of choosing the right nonisolated version of the Brieskorn module. Such nonisolated versions were e.g. looked at in the paper by van Straten [Str87]. But also Françoise in his study of normal forms was already considering

$$
F_{f}:=\frac{\Omega_{\mathbb{C}^{n}, 0}^{n}}{\{d \eta \mid d f \wedge \eta=0\}}
$$

which is again a $\mathbb{C}\{t\}$-module. For isolated singularities $F_{f}$ equals $H_{f}^{\prime \prime}$ by the de Rham lemma. (The de Rham states that wedging with $d f$ gives an exact sequence $\Omega_{\mathbb{C}^{n}, 0}^{n-2} \rightarrow \Omega_{\mathbb{C}^{n}, 0}^{n-1} \rightarrow \Omega_{\mathbb{C}^{n}, 0}^{n}$, a consequence of homological computations on the Koszul complex and the Nullstellensatz.) But for arbitrary singularities not much is known.

Due to Barlet ([BS07], prop. 3.6 together with [Bar06 thm. 4.2.2.) we 
know that for $n=2$ the latter module is free of finite rank. However, in more than two dimensions freeness of $F_{f}$ is in general not given ([BS07], remark 3.4). For normal crossings, J.-P. Françoise has computed explicit generators of the nonisolated Brieskorn module.

Proposition $2.4([\overline{F r a 82}])$. The nonisolated Brieskorn module $F_{P}$ of $P(\mathbf{x})=$ $x_{1}^{m_{1}} \ldots x_{n}^{m_{n}}$ with $m_{i} \geq 1$ can be generated as $a \mathbb{C}\{t\}$-module by $d:=\operatorname{gcd}\left(m_{1}, \ldots, m_{n}\right)$ elements. More precisely let us define $k_{i}:=m_{i} / d, i=1, \ldots, n$ and

$$
w_{i}=x_{1}^{i k_{1}-1} \ldots x_{n}^{i k_{n}-1} d x_{1} \wedge \ldots \wedge d x_{n}, \quad i=1, \ldots, d .
$$

Then the $w_{1}, \ldots, w_{d}$ generate $F_{P}$.

We make two comments which we use later. For $P=x_{1} \cdots x_{n}, F_{P}$ is generated by the single form $d^{n} \mathbf{x}=d x_{1} \wedge \ldots \wedge d x_{n}$.

The $\eta$ as appearing in the definition of $F_{P}$ can even be chosen with $\eta(0)=0$ here. We note that in the above statement convergence holds, i.e. it is not just a formal statement, although this needs a discussion which is not contained in the cited paper.

Let us consider a holomorphic function germ $f:\left(\mathbb{C}^{n}, 0\right) \rightarrow(\mathbb{C}, 0)$ with an isolated critical point at the origin. Starting from the Milnor fibration $f: X^{\prime} \rightarrow$ $S^{\prime}$ we can assemble the $i$ th complex homology of its fibres to a flat holomorphic vector bundle $H_{i}:=\bigsqcup_{t \in S^{\prime}} H_{i}\left(X_{t} ; \mathbb{C}\right) \rightarrow S^{\prime}$, the $i$ th homological Milnor bundle of $f$. Actually any holomorphic vector bundle over a noncompact Riemann surface is trivial, but instead of looking at global holomorphic sections we consider locally constant sections $\gamma: S^{\prime} \rightarrow H_{i}$. Any holomorphic $i$-form $\lambda$, which is closed on the fibre, gives rise to an integral

$$
I: S^{\prime} \rightarrow \mathbb{C}, t \mapsto \int_{\gamma(t)} \lambda
$$

Since $\gamma$ is usually multivalued (the bundle does not extend to the origin), the function $I$ is also multivalued. It can be shown by the Leray calculus ([Pha05]) that $I$ is holomorphic. This will be used later. 


\subsection{Isochoric Versal Unfoldings}

Let $f:(X, 0) \rightarrow(Y, 0)$ be a morphism of complex space germs. An unfolding of $f$ is a holomorphic map $F:(X, 0) \times(S, 0) \rightarrow(Y, 0) \times(S, 0)$ of complex space germs of the form $F(x, t)=(\widetilde{F}(x, t), t)$ and such that $\widetilde{F}(x, 0)=f(x)$. An infinitesimal unfolding of $f$ is an unfolding of $f$ as above with $S=\mathbb{T}$ where $\mathbb{T}$ denotes the double point. In the sequel we fix a (holomorphic) volume form $\Omega$ on the germ $\left(\mathbb{C}^{m}, 0\right)$. Let us discuss the notion of right left equivalence with the neccessary modifications to account for the presense of $\Omega$. We use the symbol $\mathscr{A}^{\Omega}$ here. For the general theory of unfoldings the reader may consult [Tei76], [Mar76], [Was74].

Two unfoldings $F, G$ of $f:\left(\mathbb{C}^{m}, 0\right) \rightarrow\left(\mathbb{C}^{p}, 0\right)$ with the same base space germ $(S, 0)$ are said to be $\mathscr{A}^{\Omega}$-isomorphic if there exists a commutative diagram

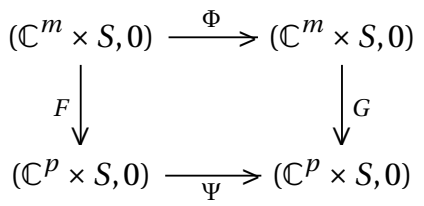

where $\Phi$ is

- an isomorphism of complex space germs,

- unfolding of the identity on $\left(\mathbb{C}^{m}, 0\right)$, i.e. it is of the form

$$
\Phi(x, s)=(\phi(x, s), s)
$$

with $\phi(x, 0)=x$ for all $x \in\left(\mathbb{C}^{m}, 0\right)$,

- $\Omega$-preserving, i.e. for every $s \in(S, 0)$ the holomorphic map

$$
\left(\mathbb{C}^{m}, 0\right) \rightarrow\left(\mathbb{C}^{m}, 0\right), x \mapsto \phi(x, s)
$$

preserves $\Omega$,

and $\Psi$ is

- an isomorphism of complex space germs, 
- an unfolding of the identity on $\left(\mathbb{C}^{p}, 0\right)$ which means that it is of the form

$$
\Psi(y, s)=(\psi(y, s), s) \quad \text { with } \psi(y, 0)=y .
$$

The trivial unfolding of $f$ with base $(S, 0)$ is the unfolding given by

$$
f \times \operatorname{id}_{S}:\left(\mathbb{C}^{m} \times S, 0\right) \rightarrow\left(\mathbb{C}^{p} \times S, 0\right) .
$$

An unfolding $F$ of $f:\left(\mathbb{C}^{m}, 0\right) \rightarrow\left(\mathbb{C}^{p}, 0\right)$ with base $(S, 0)$ is called $\mathscr{A}^{\Omega}$-trivial if it is $\mathscr{A}^{\Omega}$-isomorphic to the trivial unfolding of $f$ with base $(S, 0)$. An unfolding $F$ of $f:\left(\mathbb{C}^{m}, 0\right) \rightarrow\left(\mathbb{C}^{p}, 0\right)$ with base $(S, 0)$ is called $\mathscr{A}^{\Omega}$-versal if every unfolding $G$ of $f$ with base $\left(S^{\prime}, 0\right)$ is $\mathscr{A}^{\Omega}$-isomorphic to an unfolding obtained from $F$ by a base change $h:\left(S^{\prime}, 0\right) \rightarrow(S, 0)$. This means that for every unfolding $G$ : $\left(\mathbb{C}^{m} \times S^{\prime}, 0\right) \rightarrow\left(\mathbb{C}^{p} \times S^{\prime}, 0\right)$ of $f$ we have a commutative diagram

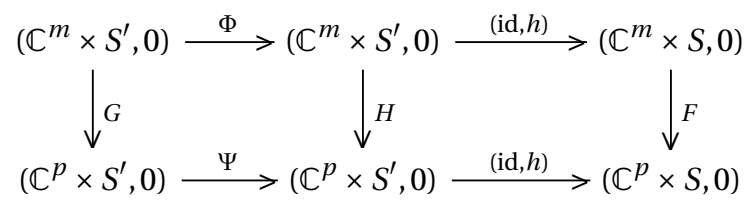

with $\Phi$ and $\Psi$ as in the definition of " $\mathscr{A}^{\Omega}$-isomorphic".

We can combine the two diagrams to a single one to get

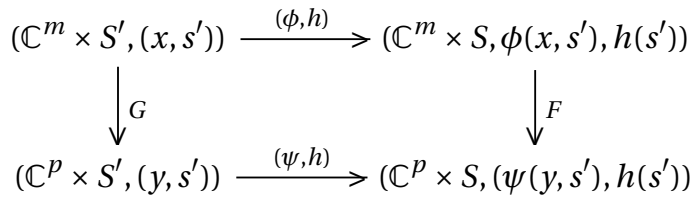

with $y=\widetilde{G}\left(x, s^{\prime}\right), \phi(\cdot, 0)=\cdot, \psi(\cdot, 0)=\cdot$ and $\phi$ volume-preserving.

If $\left(S^{\prime}, 0\right)$ is a smooth germ, then the two last diagrams are equivalent, for by the implicit function theorem $\Phi$ and $\Psi$, as constructed from the latter diagram, are isomorphisms.

For $\Omega$-isochore right equivalence of unfoldings we use the symbol $\mathscr{R}^{\Omega}$ and all the definitions above have to be modified by merely setting $\Psi:=\mathrm{id}$. 
The next proposition allows us to understand the simplest types of unfoldings, the infinitesimal ones. In general, some global property of a map induces an infinitesimal property of this map (by computing the linearization, so to say) and usually we are interested in the converse.

Proposition 2.5. Writing an infinitesimal unfolding $F$ of $f:\left(\mathbb{C}^{m}, 0\right) \rightarrow(\mathbb{C}, 0)$ in the form $\widetilde{F}=f+\epsilon g$ with $g \in \mathscr{O}_{\mathbb{C}^{m}, 0}$ we have

- F is $\mathscr{R}^{\Omega}$-trivial (as an unfolding of $f$ ) if and only if

$$
g \in\left\{\langle X, \partial f\rangle \mid L_{X} \Omega=0, X \text { hol. field on }\left(\mathbb{C}^{m}, 0\right)\right\} .
$$

- F is $\mathscr{A}^{\Omega}$-trivial (as an unfolding of f) if and only if

$$
g \in\left\{a \circ f+\langle X, \partial f\rangle \mid a \in \mathscr{O}_{\mathbb{C}, 0}, L_{X} \Omega=0, X \text { hol. field on }\left(\mathbb{C}^{m}, 0\right)\right\} .
$$

Proof. The condition for $F$ to be $\mathscr{A}^{\Omega}$-trivial is in a formula

$$
f(\phi(x, s))=\psi(\widetilde{F}(x, s), s) .
$$

We diffentiate with respect to $s$ at $s=0$ and get

$$
\begin{aligned}
\langle\partial f(x), \dot{\phi}(x, 0)\rangle & =\partial_{y} \psi \circ(\widetilde{F}(x, 0), 0)(\dot{\widetilde{F}}(x, 0))+\partial_{s} \psi(f(x), 0) \\
& =\dot{\widetilde{F}}(x, 0)+\partial_{s} \psi(f(x), 0) \\
& =g(x)+\partial_{s} \psi(f(x), 0),
\end{aligned}
$$

which is the desired relation for $\mathscr{A}^{\Omega}$. For $\mathscr{R}^{\Omega}$ we simply put $\psi(y, s)=y$ which kills the last term.

From the last proposition it follows that

$$
\begin{aligned}
\mathscr{R}_{f}^{\Omega} & :=\frac{\text { infinitesimal unfoldings of } f}{\mathscr{R}^{\Omega} \text {-trivial ones }}=\frac{\mathscr{O}_{\mathbb{C}^{m}, 0}}{\left\{\langle X, \partial f\rangle \mid L_{X} \Omega=0\right\}} \\
\mathscr{A}_{f}^{\Omega} & :=\frac{\text { inf. unfoldings of } f}{\mathscr{A}^{\Omega} \text {-trivial ones }}=\frac{\mathscr{O}_{\mathbb{C}^{m}, 0}}{\left\{f^{*} \mathscr{O}_{\mathbb{C}, 0}+\langle X, \partial f\rangle \mid L_{X} \Omega=0\right\}} .
\end{aligned}
$$

Above we have denoted by $<,>$ the standard complex bilinear pairing. Of 
course one could also simpler write $X(f)$ instead of $\langle X, \partial f\rangle$. The space $\mathscr{R}_{f}^{\Omega}$ was called by Garay the isochoric Jacobian module. We note that the denominators are not ideals in the ring $\mathscr{O}_{\mathbb{C}^{m}, 0}$, so that for example $\mathscr{R}_{f}^{\Omega}$ is not endowed (at least not in the obvious way) with a ring structure. However, we have:

Proposition 2.6. Let $f$ be an isolated singularity as above. $\mathscr{R}_{f}^{\Omega}, \mathscr{A}_{f}^{\Omega}$ carry a $\mathbb{C}\{t\}$-module structure where the multiplication is defined by

$$
a \cdot[g]:=[(a \circ f) g], \quad a \in \mathbb{C}\{t\}, g \in \mathscr{O}_{\mathbb{C}^{m}, 0} .
$$

As $\mathbb{C}\{t\}$-module we have a canonical isomorphism with the Brieskorn module of $f$ :

$$
\mathscr{R}_{f}^{\Omega} \cong H_{f}^{\prime \prime}
$$

Proof. We use the

Lemma 2.7. $d f \wedge i_{v} \Omega=\langle\nu, \partial f\rangle \Omega$

Since the formula is $\mathscr{O}_{X}$-linear in $\Omega$ we can assume for the proof of the lemma that $\Omega$ is the standard volume form. Let $v_{1}, \ldots, v_{m}$ be vectors and denote their $j$ th component (in the standard basis) by $v_{i}^{j}$. We set $d \widehat{x}^{i}=d x^{1} \wedge$ $\ldots \wedge d x^{i-1} \wedge d x^{i+1} \wedge \ldots$ and get

$$
\begin{aligned}
\left(i_{v_{1}} \Omega\right)\left(v_{2}, \ldots, v_{m}\right) & =\operatorname{det}(v) \\
& =-\sum_{j=1}^{m}(-1)^{j} v_{1}^{j} \operatorname{det}(1, j \text {-minor }) \\
& =-\sum_{j=1}^{m}(-1)^{j} v_{1}^{j} d \widehat{x}^{j}\left(v_{2}, \ldots, v_{m}\right),
\end{aligned}
$$

so that $i_{\nu} \Omega=\sum_{j=1}^{m}(-1)^{j+1} v^{j} d \widehat{x}^{j}$. Therefore,

$$
d f \wedge i_{\nu} \Omega=\sum_{i, j}(-1)^{j+1}\left(\partial_{i} f\right) v^{j} d x^{i} \wedge d \widehat{x}^{j}=\langle\nu, \partial f\rangle d x^{1} \wedge \ldots \wedge d x^{m} .
$$

This shows the lemma. The stated isomorphism of $\mathscr{R}_{f}^{\Omega}$ with the Brieskorn module is simply multiplication with $\Omega$. Under this vector space isomor- 
phism the module structure carries over to $\mathscr{R}_{f}^{\Omega}$. The assertion for $\mathscr{A}_{f}^{\Omega}$ is then immediate.

Proposition 2.8. If $f:\left(\mathbb{C}^{m}, 0\right) \rightarrow(\mathbb{C}, 0)$ is an isolated singularity with Milnor number $\mu$, then $\mathscr{R}_{f}^{\Omega}$ is a free $\mathbb{C}\{t\}$-modules of rank $\mu$.

Proof. This follows from proposition 2.6 and the the Brieskorn-Sebastiani theorem.

The following theorem is due to Gar04.

Theorem 2.9 (Isochoric Versal Unfolding Theorem). Let $f:\left(\mathbb{C}^{n}, 0\right) \rightarrow(\mathbb{C}, 0)$ be an isolated singularity and let

$$
F:\left(\mathbb{C}^{n} \times \mathbb{C}^{k}, 0\right) \rightarrow\left(\mathbb{C} \times \mathbb{C}^{k}, 0\right), \quad F(x, s)=(f(x, s), s)
$$

be an unfolding of $f$. Define the initial speeds

$$
\dot{F}_{i}:=\left.\frac{\partial f(x, s)}{\partial s_{i}}\right|_{s=0} \in \mathscr{O}_{\mathbb{C}^{n}, 0}, i=1, \ldots, k .
$$

Then the following statements are equivalent:

1. The residue classes $\left[\dot{F}_{1}\right], \ldots,\left[\dot{F}_{k}\right] \in \mathscr{A}_{f}^{\Omega}$ generate the $\mathbb{C}\{t\}$-module $\mathscr{A}_{f}^{\Omega}$.

2. The residue classes $[1],\left[\dot{F}_{1}\right], \ldots,\left[\dot{F}_{k}\right] \in \mathscr{R}_{f}^{\Omega}$ generate the $\mathbb{C}\{t\}$-module $\mathscr{R}_{f}^{\Omega}$.

3. F is $\mathscr{A}^{\Omega}$-versal (as unfolding of $f$ ).

The usual unfolding theorem (i.e. for $\mathscr{A}$-unfoldings) is the equivalence between the first and the third item. And in this case the first item requires that the residue classes of the initial speeds generate the vector space $\mathscr{R}_{f}$. Its proof has as a major ingredient a version of the Weierstraß preparation theorem, while the isochoric version needs Brieskorn modules instead.

\subsection{Proof of the Isochoric Versality Theorem}

We will provide a proof of the versality theorem for $\mathscr{A}^{\Omega}$ following the main argument of J. Martinet ([Mar76]) and the neceessary modifications to ac- 
count for $\Omega$ made by M. Garay ([Gar04].

We start with a lemma whose implication (iii) $\Rightarrow$ (i) will be the technical clue in the proof below. We will equip the spaces $\mathbb{C}^{n}, \mathbb{C}^{p+1}, \mathbb{C}^{t}$ with the coordinates $x, u$ resp. $y$.

Lemma 2.10. Let $\Omega$ be a holomorphic volume form on $\left(\mathbb{C}^{n}, 0\right)$. Let

$$
H:\left(\mathbb{C}^{p+1} \times \mathbb{C}^{n}, 0\right) \rightarrow\left(\mathbb{C}^{p+1} \times \mathbb{C}^{t}, 0\right), H(u, x)=(u, \widetilde{H}(u, x))
$$

be an unfolding in the parameters $u=\left(u_{0}, \ldots, u_{p}\right)$ and denote by $H_{0}:=\left.H\right|_{u_{0}=0}$ the $p$-parametric unfolding obtained by setting $u_{0}=0$. Then the following statements are equivalent

i) There exists a submersive holomorphic germ $h:\left(\mathbb{C}^{p+1}, 0\right) \rightarrow\left(\mathbb{C}^{p}, 0\right)$ such that $H$ is $\mathscr{A}^{\Omega}$-isomorphic to $h^{*}\left(H_{0}\right)$.

ii) There exist germs of holomorphic vector fields $X$ resp. $Y$ at the origin of $\mathbb{C}^{p+1} \times \mathbb{C}^{n}$ resp. $\mathbb{C}^{p+1} \times \mathbb{C}^{t}$ of the following form

$$
\begin{aligned}
X & =\sum_{i=1}^{n} X_{i}(u, x) \frac{\partial}{\partial x_{i}}+\frac{\partial}{\partial u_{0}}+\sum_{j=1}^{p} \xi_{j}(u) \frac{\partial}{\partial u_{j}} \\
Y & =\sum_{k=1}^{t} Y_{k}(u, y) \frac{\partial}{\partial y_{k}}+\frac{\partial}{\partial u_{0}}+\sum_{j=1}^{p} \xi_{j}(u) \frac{\partial}{\partial u_{j}}
\end{aligned}
$$

such that $\left(X_{i}(u, x)\right)_{i=1}^{n}$ is an $\Omega$-preserving vector field for every small $u$ and $X, Y$ are $H$-related, i.e. $D H(X)=Y \circ H$.

iii) There exist germs of holomorphic functions $\xi_{j}(u), X_{i}(u, x), Y_{k}(u, y)$ near the respective origins such that

$$
\frac{\partial \widetilde{H}}{\partial u_{0}}(u, x)+\sum_{j=1}^{p} \xi_{j}(u) \frac{\partial \widetilde{H}}{\partial u_{j}}(u, x)+\sum_{i=1}^{n} X_{i}(u, x) \frac{\partial \widetilde{H}}{\partial x_{i}}(u, x)=\left(Y_{l}(u, \widetilde{H}(u, x))\right)_{l=1}^{t}
$$

where $\left(X_{i}(u, x)\right)_{i=1}^{n}$ is an $\Omega$-preserving vector field on $\left(\mathbb{C}^{n}, 0\right)$ for every $u \approx$ 0 .

For the proof we refer to Martinet's article. 


\section{Milnor Number and Volume-Preserving Geometry}

Let's recall what we want to prove.

Theorem. Let $f:\left(\mathbb{C}^{n}, 0\right) \rightarrow(\mathbb{C}, 0)$ be an isolated singularity and let

$$
F:\left(\mathbb{C}^{n} \times \mathbb{C}^{k}, 0\right) \rightarrow\left(\mathbb{C} \times \mathbb{C}^{k}, 0\right), \quad F(x, s)=(f(x, s), s)
$$

be an unfolding of $f$. Define the initial speeds

$$
\dot{F}_{i}:=\left.\frac{\partial f(x, s)}{\partial s_{i}}\right|_{s=0} \in \mathscr{O}_{\mathbb{C}^{n}, 0}, i=1, \ldots, k .
$$

Then the following statements are equivalent:

1. The residue classes $\left[\dot{F}_{1}\right], \ldots,\left[\dot{F}_{k}\right] \in \mathscr{A}_{f}^{\Omega}$ generate the $\mathbb{C}\{t\}$-module $\mathscr{A}_{f}^{\Omega}$.

2. The residue classes $[1],\left[\dot{F}_{1}\right], \ldots,\left[\dot{F}_{k}\right] \in \mathscr{R}_{f}^{\Omega}$ generate the $\mathbb{C}\{t\}$-module $\mathscr{R}_{f}^{\Omega}$.

3. F is $\mathscr{A}^{\Omega}$-versal (as unfolding of $f$ ).

Proof. (3) $\Rightarrow$ (1): This is an exercise in differentiating. (1) $\Rightarrow$ (2): Trivial. (2) $\Rightarrow$ (3): Let $G:\left(\mathbb{C}^{l} \times \mathbb{C}^{n}, 0\right) \rightarrow\left(\mathbb{C}^{l} \times \mathbb{C}, 0\right)$ be an arbitrary deformation of $f$. We need to show that $G$ is $\mathscr{A}^{\Omega}$-isomorphic to the unfolding $h^{*} F$ for some germ $h:\left(\mathbb{C}^{l}, 0\right) \rightarrow\left(\mathbb{C}^{k}, 0\right)$.

To this end, we use a well-known trick by Martinet. Writing

$$
F(u, x)=(u, \widetilde{F}(u, x)) \text { and } G(\nu, x)=(\nu, \widetilde{G}(\nu, x))
$$

we form the direct sum $H$ of the unfoldings $F$ and $G$ :

$H:\left(\mathbb{C}^{k+l} \times \mathbb{C}^{n}, 0\right) \rightarrow\left(\mathbb{C}^{k+l} \times \mathbb{C}, 0\right), \quad H(u, v, x):=(u, v, \widetilde{F}(u, x)+\widetilde{G}(\nu, x)-f(x))$.

Since we substracted $f(x)$, this is again an unfolding of $f$. We obviously have $\left.H\right|_{u=0}=G$ and $\left.H\right|_{\nu=0}=F$.

The idea for the proof of the assertion, $((2) \rightarrow(3))$ is to apply lemma 2.10 , (iii) $\Rightarrow$ (i), $l$-times to get a sequence of map germs

$$
\widehat{h}:\left(\mathbb{C}^{k+l}, 0\right) \stackrel{\widehat{h}_{1}}{\longrightarrow}\left(\mathbb{C}^{k+l-1}, 0\right) \stackrel{\widehat{h}_{2}}{\longrightarrow} \ldots \rightarrow\left(\mathbb{C}^{k+1}, 0\right) \stackrel{\widehat{h}_{l}}{\longrightarrow}\left(\mathbb{C}^{k}, 0\right)
$$


such that

$$
H \text { becomes } \mathscr{A}^{\Omega} \text { - isomorphic to } \widehat{h}^{*} F \text {. }
$$

Because of $\left.H\right|_{u=0}=G$ it immediately follows that $G$ is $\mathscr{A}^{\Omega}$-isomorphic to $h^{*} F$ with $h:=\left.\hat{h}\right|_{u=0}:\left(\mathbb{C}^{l}, 0\right) \rightarrow\left(\mathbb{C}^{k}, 0\right)$. Hence, the assertion $(2) \Rightarrow(3)$ would be proved.

The first step is to use (2.10), (iii) $\Rightarrow$ (i), (with $v_{1}$ instead of $u_{0}$ and $v_{1}, \ldots, v_{l}$, $u_{1}, \ldots, u_{k}$ instead of $u_{0}, \ldots, u_{p}$ ). To do so, we need to show the validity of an equation of the form

$$
\begin{aligned}
& \frac{\partial \widetilde{H}}{\partial \nu_{1}}(u, v, x)+\sum_{j=1}^{k} \xi_{j}(u, v) \frac{\partial \widetilde{H}}{\partial u_{j}}(u, v, x)+\quad(* *) \\
+ & \sum_{j=2}^{l} \widehat{\xi}_{j}(u, v) \frac{\partial \widetilde{H}}{\partial v_{j}}(u, v, x)+\sum_{i=1}^{n} X_{i}(u, v, x) \frac{\partial \widetilde{H}}{\partial x_{i}}(u, v, x)=Y(u, v, \widetilde{H}(u, v, x)) .
\end{aligned}
$$

If such an equation holds (on the level of germs, with $\left(X_{i}\right)_{i=1}^{n}$ relatively $\Omega$ isochore), then, according to the lemma, $H$ is $\mathscr{A}^{\Omega}$-isomorphic to the unfolding $\hat{h}_{1}^{*}\left(\left.H\right|_{\nu_{1}=0}\right)$ for some germ $\hat{h}_{1}:\left(\mathbb{C}^{k} \times \mathbb{C}^{l}, 0\right) \rightarrow\left(\mathbb{C}^{k} \times \mathbb{C}^{l-1}, 0\right)$. By applying this procedure recursively (to the pair $\left(\left.H\right|_{\nu_{1}=0},\left.H\right|_{\nu_{1}=0, \nu_{2}=0}\right)$ instead of $\left(H,\left.H\right|_{\nu_{1}=0}\right)$ in the next step, etc.), the proof of $\left({ }^{*}\right)$ is then completed.

(Remark. Here one has to show that for instance, if $H$ is $\mathscr{A}^{\Omega}$-isomorphic to $\left.\widehat{h}_{1}^{*} H\right|_{\nu_{1}=0}$ and if $\left.H\right|_{\nu_{1}=0}$ is $\mathscr{A}^{\Omega}$-isomorphic to $\left.\widehat{h}_{2}^{*} H\right|_{\nu_{1}=\nu_{2}=0}$, then $H$ is $\mathscr{A}^{\Omega}$. isomorphic to $\left(\widehat{h}_{2} \circ \widehat{h}_{1}\right)^{*}\left(\left.H\right|_{\nu_{1}=v_{2}=0}\right)$.)

We now verify the last equation $\left.{ }^{* *}\right)$. Consider

$$
\mathscr{R}_{\widetilde{H}}^{\Omega, \text { par }}=\frac{\mathscr{O}_{\left(\mathbb{C}^{k+l+n}, 0\right)}}{\left\{<X(u, v, x), \partial_{x} \widetilde{H}>\mid L_{X} \Omega=0\right\}},
$$

a parametric version of the isochoric Jacobian module. Similar to the last section multiplication by the volume form shows that this is isomorphic to the Greuel-Brieskorn module of $H$, a $\mathbb{C}\{z, u, v\}$-module (via concatenation with $H)$. Now $H^{-1}(0)=\left\{(u, v, x) \in \mathbb{C}^{k} \times \mathbb{C}^{l} \times \mathbb{C}^{n} \mid u=0, v=0, f(x)=0\right\} \cong f^{-1}(0)$ 
and from proposition 1.8 we see that $H$ is an isolated complete intersection singularity, therefore by theorem $2.3 \mathscr{R}_{\widetilde{H}}^{\Omega, \text { par }}$ is free of rank $\mu(H)=\mu(f)$.

The equation in question $\left.{ }^{* *}\right)$ can be written simply as the question whether the residue class

$$
\left[\frac{\partial \widetilde{H}}{\partial \nu_{1}}\right] \in \mathscr{R}_{\widetilde{H}}^{\Omega, \text { par }}
$$

belongs to the $\mathbb{C}\{z, u, v\}$-submodule of $\mathscr{R}_{\widetilde{H}}^{\Omega, \text { par }}$ generated by the classes

$$
[1],\left[\partial_{u_{1}} \widetilde{H}\right], \ldots,\left[\partial_{u_{k}} \widetilde{H}\right],\left[\partial_{v_{2}} \widetilde{H}\right], \ldots,\left[\partial_{v_{l}} \widetilde{H}\right] .
$$

We assert that this submodule is indeed the whole module.

Let $\mathfrak{m}_{1}$ be the maximal ideal in $\mathbb{C}\{z\}$ and $\mathfrak{m}_{k+l+1}$ be the maximal ideal in $\mathbb{C}\{z, u, v\}$. Denote by

$$
\begin{aligned}
\pi: \mathscr{R}_{\widetilde{H}}^{\Omega, \text { par }} & \rightarrow \frac{\mathscr{R}_{\widetilde{H}}^{\Omega, \text { par }}}{\mathfrak{m}_{k+l+1} \mathscr{R}_{\widetilde{H}}^{\Omega, \mathrm{par}}} \\
\pi \mid: \mathscr{R}_{f}^{\Omega} & \rightarrow \frac{\mathscr{R}_{f}^{\Omega}}{\mathfrak{m}_{1} \mathscr{R}_{f}^{\Omega}}
\end{aligned}
$$

the canonical projections. Note that $\pi|=\pi|_{u=v=0}$.

Now since $\mathscr{R}_{\widetilde{H}}^{\Omega, p a r}$ is a $\mathbb{C}\{z, u, v\}$-module of finite type, showing that the above mentioned submodule is the whole $\mathscr{R}_{\widetilde{H}}^{\Omega, \text { par }}$, is therefore equivalent by the Nakayama lemma to showing that $\pi$, restricted to the submodule, is again surjective. 
Now look at the following commutative diagram

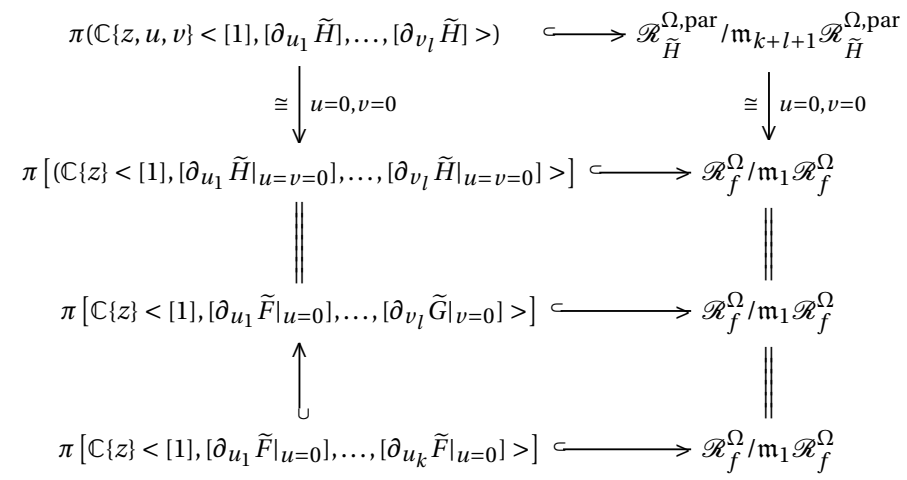

The hooked arrows are inclusions, and the upper map to the right is the isomorphism $\mathbb{C}^{\mu} \cong \mathbb{C}^{\mu}$ where $\mu=\mu(H)=\mu(f)$. By hypothesis (1) in the statement of the versality theorem, the module $\mathbb{C}\{z\}<[1],\left[\left.\partial_{u_{1}} \widetilde{F}\right|_{u=0}\right], \ldots,\left[\left.\partial_{u_{k}} \widetilde{F}\right|_{u=0}\right]$ is equal to $\mathscr{R}_{f}^{\Omega}$, hence the last line is an equality, so is the first. This completes the proof.

\subsection{Vey's Isochoric Morse Lemma}

Let $f:\left(\mathbb{C}^{n}, 0\right) \rightarrow(\mathbb{C}, 0)$ be a holomorphic function germ which is critical and Morse at the origin. The classical Morse lemma asserts that there is a coordinate transformation $\Phi \in \operatorname{Aut}\left(\mathbb{C}^{n}, 0\right)$ such that

$$
f \circ \Phi\left(x_{1}, \ldots, x_{n}\right)=x_{1}^{2}+\ldots+x_{n}^{2} .
$$

If one stipulates that the coordinate change has to be volume-preserving one has the "lemme de Morse isochore" by J. Vey ([Vey77]):

Theorem 2.11 (Volume-Preserving Morse Lemma). Let $f:\left(\mathbb{C}^{n}, 0\right) \rightarrow(\mathbb{C}, 0)$ be a critical Morse singularity. Then there exists a volumepreserving automorphism $\Phi \in \operatorname{Aut}\left(\mathbb{C}^{n}, 0\right)$ and a holomorphic germ $\Psi:(\mathbb{C}, 0) \rightarrow$ $(\mathbb{C}, 0)$ such that

$$
f \circ \Phi=\Psi \circ Q_{f} .
$$

Here $Q_{f}$ denotes the quadratic part of $f$ at the origin. It is possible to demand 
$\Psi(t)=t+O\left(t^{2}\right)$ and with this requirement $\Psi$ is uniquely determined by $f$.

The rest of this section is devoted to a proof of this theorem. The existence proof will make use of the isochoric unfolding theorem by Garay when the Milnor number is one (Gar07]). For the uniqueness of $\Psi$ we discuss the linear problem and integrals over cycles in the Milnor fibre of $f$.

We start by looking at the linear problem. That is, we consider the group action

$$
\mathrm{SL}_{n} \mathbb{C} \times \operatorname{Sym}_{n} \mathbb{C} \rightarrow \operatorname{Sym}_{n} \mathbb{C}, \quad(S, A) \mapsto S^{t} A S .
$$

Here $\operatorname{Sym}_{n} \mathbb{C}$ is the vector space of complex, symmetric $(n \times n)$-matrices. The following easy proposition classifies the orbits of the group action.

Proposition 2.12. Two symmetric matrices $A, B \in \mathbb{C}^{n \times n}$ are equivalent (under the congruence action of $\mathrm{SL}_{n} \mathbb{C}$ ) if and only if either

- they have both the same rank $k<n$, or

- they have both the full rank $k=n$ and $\operatorname{det}(A)=\operatorname{det}(B)$.

Proof. The neccessity of the rank and determinant conditions is clear. So let $A$ and $B$ have the same rank $k$. Since $A$ is symmetric of rank $k$, by Sylvester there is an invertible matrix $\widetilde{S} \in \mathrm{GL}_{n} \mathbb{C}$ such that $\widetilde{S}^{t} A \widetilde{S}$ is of the form

$$
\left(\begin{array}{cc}
I_{k} & 0 \\
0 & 0
\end{array}\right)
$$

where $I_{k}$ is the $k \times k$-identity matrix $(0 \leq k \leq n)$. Now we can choose any of the $n$ values of the root $\lambda=\sqrt[n]{\operatorname{det}(\widetilde{S})}$ and with the definition

$$
S:=\frac{1}{\lambda} \widetilde{S} \quad \text { we get } \quad S^{t} A S=\frac{1}{\lambda^{2}}\left(\begin{array}{cc}
I_{k} & 0 \\
0 & 0
\end{array}\right), \quad \operatorname{det}(S)=1 .
$$

If we write $B$ in the same manner, we are left to check when the resulting matrices

$$
\frac{1}{\lambda^{2}}\left(\begin{array}{cc}
I_{k} & 0 \\
0 & 0
\end{array}\right) \quad \text { and } \quad \frac{1}{\mu^{2}}\left(\begin{array}{cc}
I_{k} & 0 \\
0 & 0
\end{array}\right)
$$

are congruent under $\mathrm{SL}_{n} \mathbb{C}$. 
That's why we look for a matrix $S=\left(\begin{array}{ll}E & F \\ G & H\end{array}\right)$ with $\operatorname{det}(S)=1$ and $E \in \mathbb{C}^{k \times k}, F \in$ $\mathbb{C}^{k \times(n-k)}$, etc. such that

$$
S^{t} \frac{1}{\lambda^{2}}\left(\begin{array}{cc}
I_{k} & 0 \\
0 & 0
\end{array}\right) S=\frac{1}{\mu^{2}}\left(\begin{array}{cc}
I_{k} & 0 \\
0 & 0
\end{array}\right) .
$$

This is equivalent to

$$
\begin{aligned}
\frac{1}{\mu^{2}}\left(\begin{array}{cc}
I_{k} & 0 \\
0 & 0
\end{array}\right) & =\left(\begin{array}{cc}
E^{t} & G^{t} \\
F^{t} & H^{t}
\end{array}\right) \frac{1}{\lambda^{2}}\left(\begin{array}{cc}
I_{k} & 0 \\
0 & 0
\end{array}\right)\left(\begin{array}{cc}
E & F \\
G & H
\end{array}\right) \\
& =\frac{1}{\lambda^{2}}\left(\begin{array}{ll}
E^{t} & G^{t} \\
F^{t} & H^{t}
\end{array}\right)\left(\begin{array}{cc}
E & F \\
0 & 0
\end{array}\right) \\
& =\frac{1}{\lambda^{2}}\left(\begin{array}{ll}
E^{t} E & E^{t} F \\
F^{t} E & F^{t} F
\end{array}\right)
\end{aligned}
$$

which means $E^{t} E=\frac{\lambda^{2}}{\mu^{2}} I_{k}$ and $F^{t} E=0$. So $E$ is invertible and $F=0$. Then by a well-known theorem for computing the determinant of a block-matrix with $F=0$ we get

$$
\operatorname{det}\left(\begin{array}{ll}
E & F \\
G & H
\end{array}\right)=\operatorname{det}(E) \operatorname{det}(H) .
$$

In order to get congruence under $S \in \mathrm{SL}_{n} \mathbb{C}$, the matrices $E$ and $H$ have to be chosen with

$\operatorname{det}(E)^{2}=\left(\frac{\lambda}{\mu}\right)^{2 k}$ and $\operatorname{det}(H)=1 / \operatorname{det}(E), \quad$ while $F=0$ and $G$ arbitrary.

If $k<n$, such matrices can be easily found, e.g. by choosing $E$ and $H$ as multiples of the identity matices of the corresponding dimension and such that $\left({ }^{*}\right)$ holds. This shows the first part of the proposition.

However, when the rank $k$ of $A$, is equal to $n$, then there is no $H$ which can force the determinant of $S$ to be equal to one and so in this case we really need $\operatorname{det}(E)=1$ (in fact $S=E$ ). Using the above notation, it suffices to show that we can always find such $S \in \mathrm{SL}_{n} \mathbb{C}$ with $S^{t} \frac{1}{\lambda^{2}} S=\frac{1}{\mu^{2}} I$. By the very definition of $\lambda$ and $\mu$ we have $\operatorname{det}(A)=\frac{1}{\lambda^{2 n}}$ and $\operatorname{det}(B)=\frac{1}{\mu^{2 n}}$. Now since 
$\operatorname{det}(A)=\operatorname{det}(B)$, we get $\left(\lambda^{2} / \mu^{2}\right)^{n}=1$. Thus the desired result follows from the next lemma with $c:=\frac{\lambda^{2}}{\mu^{2}}$.

Lemma 2.13. There always exists a solution $S \in \mathrm{SL}_{n} \mathbb{C}$ of the equation $S^{t} S=$ cI for any $n$th root of unity $c$.

Proof. Choose an $n$th root $\lambda$ of $c$. Since $c^{n}=1$ we have $\lambda^{n}=1$ or $\lambda^{n}=-1$. In the first case put $S=\lambda I$ where $I$ is the $n \times n$ identity matrix; in the second case put $S=\lambda \operatorname{diag}(-1,1, \ldots, 1)$. Then in both cases $S^{t} S=\lambda^{2} I=c I$ and $\operatorname{det}(S)=1$.

This completes the proof of proposition 2.12

Given two holomorphic germs $f^{A}(x)=x^{t} A x+O\left(\|x\|^{2}\right)$ and $f^{B}(x)=x^{t} B x+$ $O\left(\|x\|^{2}\right)$ for some $A, B \in \mathbb{C}^{n \times n}$ and assume that there exist holomorphic germs $\Phi:\left(\mathbb{C}^{n}, 0\right) \rightarrow\left(\mathbb{C}^{n}, 0\right)$ and $\Psi:(\mathbb{C}, 0) \rightarrow(\mathbb{C}, 0)$ such that

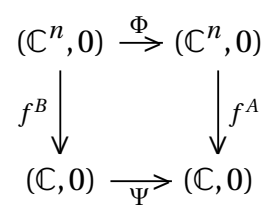

commutes. Then we have $f^{A}(\Phi(x))=\Psi\left(f^{B}(x)\right)$ and by comparing the quadratic parts we can deduce, using $S:=D \Phi(0)$, that

$$
S^{t} A S=\Psi^{\prime}(0) B .
$$

This makes clear the role of $\Psi^{\prime}(0)$ in Vey's theorem: If $\operatorname{det}(A)=\operatorname{det}(B) \neq 0$ (as is the case in the statement of theorem 2.11, we have $\Psi^{\prime}(0)^{n}=1$. By lemma 2.13 we cannot get rid of this nonuniqueness of $\Psi^{\prime}(0)$, so we have to put it as a requirement.

Lemma 2.14. Let $f:\left(\mathbb{C}^{n}, 0\right) \rightarrow \mathbb{C}$ be critical and Morse at the origin. Then for any s-parametric unfolding $G$ of $f$ there is a commutative diagram 


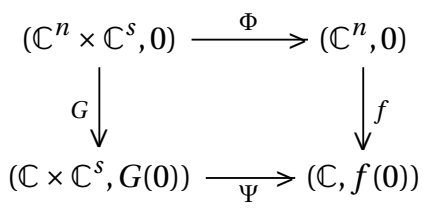

with $\Phi$ a $\Omega$-preserving and holomorphic germ and $\Psi$ a holomorphic germ.

Proof. We can consider $f$ itself as an unfolding of $f$ (i.e. $k=0$ in the versality theorem). Since $f$ is Morse and critical, its Milnor number is $\mu=1$ so that $\mathscr{R}_{f}^{\Omega} \cong \mathbb{C}\{t\}^{1}$ by the proposition 2.8 . Therefore we can apply the $\mathscr{A}^{\Omega}$-versality theorem, (2) $\Rightarrow$ (3), to $F:=f$ which shows the assertion.

The above lemma makes it now possible to prove the existence of $\Psi$ in Vey's lemma. Put $f_{t}:=(1-t) Q_{f}+t f, t \in \mathbb{C}$. Then for any value $t_{i} \in[0,1]$, by abuse of notation, the germ $\left.f_{s}\right|_{s \approx t_{i}}$ is an unfolding of $f_{t_{i}}$. To be more precise, by $\left.f_{s}\right|_{s \approx t_{i}}$ we denote the first map in the unfolding $F_{i}$ of $f_{t_{i}}$ :

$$
F_{i}:\left(\mathbb{C}^{n}, 0\right) \times\left(\mathbb{C}, t_{i}\right) \rightarrow(\mathbb{C}, 0) \times\left(\mathbb{C}, t_{i}\right), \quad(x, s) \mapsto\left(f_{s}(x), s\right) .
$$

The Hessian matrix of $f_{t}$ at the origin in $\mathbb{C}^{n}(!)$ is

$$
\operatorname{Hess}_{0}\left(f_{t}\right)=(1-t) \operatorname{Hess}_{0}\left(Q_{f}\right)+t \operatorname{Hess}_{0}(f)=\operatorname{Hess}_{0}(f) .
$$

Hence each germ $f_{t_{i}}, t_{i} \in[0,1]$, has the origin as a critical nondegenerate point so that we can apply lemma 2.14 for every $t_{i} \in[0,1]$ to get the following commutative diagram

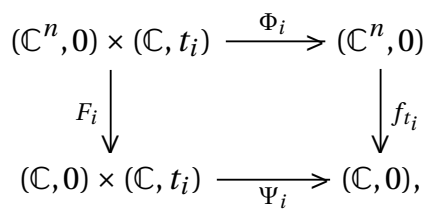

where the $\Phi_{i}$ is relative isochore. The domains of definition of the maps involved, give, when $t_{i}$ varies over [0,1], an open cover of the compact set $[0,1]$, so that we may assume that we have a sequence $0=t_{0}<t_{1}<\ldots<t_{N}=$ 1 of points in $[0,1]$ and a commutative diagram 


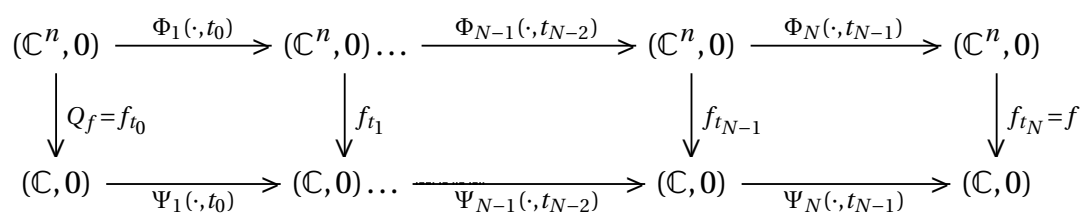

Hence we obtain a commutative diagram, as on the right, with $\Phi$ holomorphic and isochore and $\Psi$ holomorphic.

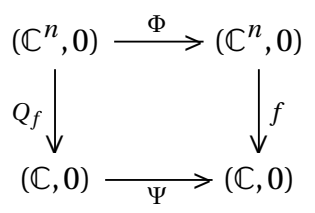

Since $\Phi$ is isochore, it is a local biholomorphism by the inverse function theorem. It remains to show that $\Psi(t)=t+O\left(t^{2}\right)$. Equation 2.12, applied to our situation $\left(\operatorname{since}^{\operatorname{det}}\left(\operatorname{Hess}_{0}(f)\right)=\operatorname{det}\left(\operatorname{Hess}_{0}\left(Q_{f}\right)\right)\right)$, obviously gives us $\Psi^{\prime}(0)^{n}=1$. If $\Psi^{\prime}(0)=1$, the proof is completed. If this does not hold we apply the previous considerations in linear algebra to $c:=\frac{1}{\Psi^{\prime}(0)}$ to obtain a commutative diagram

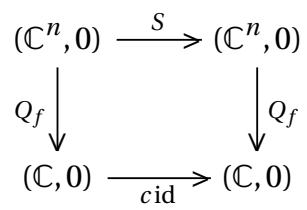

where $S$ is a volume-preserving matrix. To this diagram we compose the previous one to get an overall $\Psi$ which is finally normed $\left(\Psi^{\prime}(0)=1\right)$.

Let us prove that $\Psi$ is quite unique. Let $t \mapsto \delta_{f}(t) \in H_{n-1}(X(t) ; \mathbb{C})$ be a locally constant section of the homological Milnor fibration of $f$. This gives us a cycle in the Milnor fibre $X(t)$ of $f$ over each $t \approx 0, t \neq 0$, and well determined by $f$ up to sign. Let $\lambda$ be any holomorphic $n-1$-form which is primitive for the standard volume-form $\Omega$.

The association

$$
I_{f}:(\mathbb{C}, 0) \rightarrow \mathbb{C}, I_{f}(t):=\int_{\delta_{f}(t)} \lambda \mid X(t)
$$


defines a holomorphic multivalued function with monodromy $(-1)^{n}$. For a given $f, I_{f}$ is only defined up to a global sign, which corresponds to the inital choice of a vanishing cycle.

Proposition 2.15. Let $Q(x)=x^{t} A x$ be any nondegenerate quadratic form (in $n$ complex variables). Then

$$
\int_{\delta_{Q}(t)} \lambda=\frac{\sqrt{t}^{n}}{\sqrt{\operatorname{det}(A)}} \frac{\pi^{n / 2}}{\Gamma\left(\frac{n}{2}+1\right)}
$$

as multivalued germs in $\left(\mathbb{C}^{*}, 0\right)$.

Note that the there are two choices of $\sqrt{\operatorname{det}(A)}$ and the monodromy of $\sqrt{t}^{n}$ is precisely $(-1)^{n}$.

Proof. Let $S \in \mathrm{GL}_{n} \mathbb{C}$ be such that $A=S^{t} S$ (note that $A$ is complex and symmetric). Then $\Phi \in \operatorname{Aut}\left(\mathbb{C}^{n}, 0\right)$ with $x=\Phi(y):=S^{-1} y$ transforms $Q(x)=$ $x^{t} A x$ into Morse coordinates $\left(\Phi^{*} Q\right)(y)=y^{t} y$. Denote by $\delta_{I}$ the vanishing cycle of $y \rightarrow y^{t} y$ and recall e.g. from [Lam81], p. 37 a concrete coordinate description using the real sphere $S^{n-1}$. We get

$$
\begin{aligned}
\int_{\delta_{Q}(t)} \lambda & =\int_{\Phi_{*} \delta_{I}(t)} \lambda \\
& =\int_{\delta_{I}(t)} \Phi^{*} \lambda \\
& =\int_{y \in \sqrt{t} S^{n-1}} \Phi^{*}(\lambda) \\
& =\int_{y \in \sqrt{t} B^{n}} \Phi^{*} d^{n} \mathbf{x} \\
& =\int_{y \in \sqrt{t} B^{n}} \operatorname{det} D \Phi(y) d y \\
& =\frac{1}{\operatorname{det}(S)} \int_{y \in \sqrt{t} B^{n}} d y \\
& =\frac{\sqrt{t}}{\operatorname{det}(S)} \operatorname{Vol}\left(B^{n}\right) \\
& =\frac{\sqrt{t}}{\sqrt{\operatorname{det}(A)}} \frac{\pi^{n / 2}}{\Gamma\left(\frac{n}{2}+1\right)}
\end{aligned}
$$


Proposition 2.16. Let $f:\left(\mathbb{C}^{n}, 0\right) \rightarrow(\mathbb{C}, 0)$ be a critical Morse singularity with quadratic part $Q$. Assume that we know $f \circ \Phi=\Psi \circ Q$ for some $\Phi \in \operatorname{Aut}\left(\mathbb{C}^{n}, 0\right)$. Let $\lambda$ be a holomorphic primitive of the standard volume form $\Omega$ on $\mathbb{C}^{n}$. Then

$$
\int_{\delta_{f}(t)} \lambda=\frac{\pi^{n / 2}}{\Gamma\left(\frac{n}{2}+1\right)} \frac{\left(\Psi^{-1}(t)\right)^{n / 2}}{\sqrt{\operatorname{det}(Q)}}
$$

as multivalued germs in $\left(\mathbb{C}^{*}, 0\right)$.

Proof. It is essential to note that $\Phi_{*}$ maps $\delta_{Q}(t)$ to $\delta_{f}(\Psi(t))$. This follows since $H_{n-1}\left(\operatorname{Mil}_{Q}(t) ; \mathbb{Z}\right) \cong=\mathbb{Z}$ and the fact that $\delta_{Q}(t)$ is a generator of this group and so must be mapped by $\Phi_{*}$ to a generator of $H_{n-1}\left(\operatorname{Mil}_{f}(\Psi(t)) ; \mathbb{Z}\right) \cong$ $\mathbb{Z}^{\mu}=\mathbb{Z}$. From this we obtain

$$
\begin{aligned}
\int_{\delta_{f}(\Psi(t))} \lambda & =\int_{(\Phi)_{*} \delta_{Q}(t)} \lambda \\
& =\int_{\delta_{Q}(t)}(\Phi)^{*} \lambda
\end{aligned}
$$

Since $\Phi$ preserves $d \lambda$ and exact forms are irrelevant for integration we get together with proposition 2.15

$$
\int_{\delta_{f}(\Psi(t))} \lambda=\frac{\pi^{n / 2}}{\Gamma\left(\frac{n}{2}+1\right)} \frac{t^{n / 2}}{\sqrt{\operatorname{det}(Q)}}
$$

as asserted.

The last proposition gives us a geometric interpretation of Vey's $\Psi$-function. It measures the volume in each fibre.

Corollary 2.17. $\Psi_{f}$ is unique up to the multiplication with an nth root of unity. 


\subsection{An Isochoric Normal Form for Reduced Normal Crossings}

This section is based on [Sza12a]. Using techniques from [Fra82 and [Fra78 we will prove the following theorem.

Theorem 2.18. Consider a holomorphic germ $f:\left(\mathbb{C}^{n}, 0\right) \rightarrow(\mathbb{C}, 0)$ that is right equivalent to the product of all coordinates: $f \sim x_{1} \cdots x_{n}$. Then there exists a volume-preserving automorphism $\Phi:\left(\mathbb{C}^{n}, 0\right) \rightarrow\left(\mathbb{C}^{n}, 0\right)$ and an automorphism $\Psi:(\mathbb{C}, 0) \rightarrow(\mathbb{C}, 0)$ such that

$$
f(\Phi(\mathbf{x}))=\Psi\left(x_{1} \cdots x_{n}\right) .
$$

$\Psi$ is uniquely determined by $f$ up to a sign.

Lemma 2.19. For $v, w \in \mathbb{C}^{n}$ (written as column vectors) and $a, b \in \mathbb{C}$ we have

$$
\operatorname{det}\left(a I+b v w^{t}\right)=a^{n-1}\left(a+b v^{t} w\right) .
$$

This follows immediately from the next lemma.

Lemma 2.20. For $v, w \in \mathbb{C}^{n}$ (written as column vectors) and $\lambda \in \mathbb{C}$ we have

$$
\operatorname{det}\left(\lambda I-v w^{t}\right)=\lambda^{n-1}\left(\lambda-v^{t} w\right) .
$$

Proof. We can assume $v \neq 0$ and $w \neq 0$. Since we clearly have $\operatorname{det}\left(\lambda I-v w^{t}\right)=$ $\prod_{i=1}^{n}\left(\lambda-\lambda_{i}\right)$, where $\lambda_{1}, \ldots, \lambda_{n}$ denote the eigenvalues of $v w^{t}$, it suffices for the assertion to determine all eigenvalues of $v w^{t}$ together with their algebraic multiplicity.

The eigenvalue zero occurs if and only if there is a nonzero vector $x$ with $v w^{t} x=0$. Now $w^{t} x$ is a number and we conclude (since $v \neq 0$ ) that it must be zero then, i.e. $x$ is perpendicular to $w$. Since $w \neq 0$, the dimension of the kernel of $v w^{t}$ is therefore $n-1$, the algebraic multiplicity of the zero eigenvalue hence $n-1$ or $n$.

Now assume that there exists a nonzero eigenvalue $\lambda$ of $v w^{t}$. Then there is $x \neq 0$ with $v w^{t} x=\lambda x$. It follows $w^{t} v w^{t} x=\lambda w^{t} x$, hence $w^{t} x\left(w^{t} v-\lambda\right)=0$. If $w^{t} x$ would be zero, then $0=v w^{t} x=\lambda x$, hence either $\lambda=0$ or $x=0$, giving 


\section{Milnor Number and Volume-Preserving Geometry}

a contradiction. So $v^{t} w=\lambda$ is the only possible nonzero eigenvalue of $v w^{t}$. And vice versa: if $v^{t} w \neq 0$, then this is a nonzero eigenvalue with eigenspace $\mathbb{C}<v>$.

Hence, if $v^{t} w=0$ then only zero eigenvalues occur and we have $\operatorname{det}(\lambda I-$ $\left.v w^{t}\right)=\lambda^{n}$ and if $v^{t} w \neq 0$ then precisely one nonzero eigenvalue occurs and $\operatorname{det}\left(\lambda I-v w^{t}\right)=\lambda^{n-1}\left(\lambda-v^{t} w\right)$.

Lemma 2.21 (Main Lemma).

Let $P$ be quasihomogeneous of type $\left(w_{1}, \ldots, w_{n} ; N\right)$. Let $u \in \mathscr{O}_{\mathbb{C}, 0}$ with $u(0) \neq 0$. Then the map

$$
A:\left(\mathbb{C}^{n}, 0\right) \rightarrow\left(\mathbb{C}^{n}, 0\right), \quad \mathbf{x} \mapsto\left(u(P(\mathbf{x}))^{w_{1}} x_{1}, \ldots, u(P(\mathbf{x}))^{w_{n}} x_{n}\right)
$$

defines an automorphism of $\left(\mathbb{C}^{n}, 0\right)$ with the following properties:

a) There exists a unique $v \in \mathscr{O}_{\mathbb{C}, 0}$ such that the inverse map $A^{-1}$ is given by

$$
\mathbf{z} \mapsto\left(\nu(P(\mathbf{z}))^{w_{1}} z_{1}, \ldots, v(P(\mathbf{z}))^{w_{n}} z_{n}\right) .
$$

Furthermore $v(0) \neq 0$.

b) With this $v$, the Jacobian determinant of $A^{-1}$ is given by

$$
\operatorname{det}\left(D A^{-1}(\mathbf{z})\right)=\left.\left(v(P)^{w}+\frac{N}{w} P \frac{d}{d P} v(P)^{w}\right)\right|_{P=P(\mathbf{z})} .
$$

Here we have put $w:=w_{1}+\ldots+w_{n}$.

c) If we denote the assignment $u \mapsto v$ by $E: \operatorname{Units}\left(\mathscr{O}_{\mathbb{C}, 0}\right) \rightarrow \operatorname{Units}\left(\mathscr{O}_{\mathbb{C}, 0}\right)$, then $E \circ E=$ id.

Proof. The assignment

$$
A: \mathbf{x} \mapsto \mathbf{z}:=\left(u(P(\mathbf{x}))^{w_{1}} x_{1}, \ldots, u(P(\mathbf{x}))^{w_{n}} x_{n}\right)
$$

is an automorphism of $\left(\mathbb{C}^{n}, 0\right)$ since its Jacobian at the origin is regular:

$$
D A(0)=\left(\begin{array}{ccc}
u(0)^{w_{1}} & 0 & \ldots \\
& \ldots & \\
\ldots & 0 & u(0)^{w_{n}}
\end{array}\right) .
$$


It is clear that the inverse of $A$ is of the form $A^{-1}: \mathbf{z} \rightarrow\left(\tilde{v}_{1}(\mathbf{z}) z_{1}, \ldots, \tilde{v}_{n}(\mathbf{z}) z_{n}\right)$ for some $\tilde{v} \in \mathscr{O}_{\mathbb{C}^{n}, 0}$.

(In fact, if we write $x_{i}=x_{i}(\mathbf{z})$ for the components of $A^{-1}(\mathbf{z})$, then $z_{i}=$ $u(P(\mathbf{x}(\mathbf{z})))^{w_{i}} x_{i}(\mathbf{z})$, so that $z_{i}$ must divide $x_{i}(\mathbf{z})$.)

In the sequel we are going to show that it is even of the form

$$
\mathbf{z} \mapsto\left(\nu(P(\mathbf{z}))^{w_{1}} z_{1}, \ldots, v(P(\mathbf{z}))^{w_{n}} z_{n}\right)
$$

for some $v \in \mathscr{O}_{\mathbb{C}, 0}$ ! We also show that $v$ is uniquely determined by $u$ and that $v(0) \neq 0$.

Let $\mathbf{z}=A(\mathbf{x})$. From

$$
\begin{aligned}
\mathbf{z} & =\left(u(P(\mathbf{x}))^{w_{1}} x_{1}, \ldots, u(P(\mathbf{x}))^{w_{n}} x_{n}\right) \\
& =\left(u(P(\mathbf{x}))^{w_{1}} \tilde{v}_{1}(\mathbf{z}) z_{1}, \ldots, u(P(\mathbf{x}))^{w_{n}} \tilde{v}_{n}(\mathbf{z}) z_{n}\right)
\end{aligned}
$$

we conclude that

$$
1=u(P(\mathbf{x}))^{w_{i}} \tilde{v}_{i}(\mathbf{z}) \text { for } i=1, \ldots, n .
$$

Hence for the function $\hat{v} \in \mathscr{O}_{\mathbb{C}^{n}, 0}$ defined by

$$
\hat{v}(\mathbf{z}):=\frac{1}{u\left(P\left(A^{-1}(\mathbf{z})\right)\right)},
$$

we have

$$
\tilde{v}_{i}(\mathbf{z})=\hat{v}(\mathbf{z})^{w_{i}} \text { for all } i .
$$

Now let us show that the function $\hat{v}$ factors through $P(\mathbf{z})$. First we rewrite its defining equation

$$
\begin{aligned}
1 & =u(P(\mathbf{x})) \hat{v}(\mathbf{z}) \\
& =u\left(P\left(\tilde{v}_{1}(\mathbf{z}) z_{1}, \ldots, \tilde{v}_{n}(\mathbf{z}) z_{n}\right)\right) \hat{v}(\mathbf{z}) \\
& =u\left(P\left(\hat{v}(\mathbf{z})^{w_{1}} z_{1}, \ldots, \hat{v}(\mathbf{z})^{w_{n}} z_{n}\right)\right) \hat{v}(\mathbf{z}) \\
& =u\left(P\left(z_{1}, \ldots, z_{n}\right) \hat{v}(\mathbf{z})^{N}\right) \hat{v}(\mathbf{z}) .
\end{aligned}
$$

To see factorization through $P$, we apply twice the implicit function theorem as follows. 
1. The implicit equation $u\left(v^{N} t\right) v=1$ for $v$ has a unique local solution $v=v(t):(\mathbb{C}, 0) \rightarrow(\mathbb{C}, 1 / u(0))$. Indeed, the point $(t=0, v=1 / u(0))$ is a solution and the derivative after $v$ is nonzero at this point:

$$
\left.\partial_{\nu}(u(0) v)\right|_{\nu=1 / u(0)}=u(0) \neq 0 .
$$

2. The implicit equation $u\left(V^{N} P(\mathbf{z})\right) V=1$ for $V$ has a unique local solution $V=V(\mathbf{z}):\left(\mathbb{C}^{n}, 0\right) \rightarrow(\mathbb{C}, 1 / u(0))$. Indeed, the point $(\mathbf{z}=0, V=$ $1 / u(0))$ is a solution and the derivative after $V$ at this point is nonzero:

$$
\left.\partial_{V}(u(0) V)\right|_{V=1 / u(0)}=u(0) \neq 0 .
$$

Now by the first item (only existence is used), $v(P(\mathbf{z}))$ fulfils $v(P(0))=$ $1 / u(0)$ and solves the equation $u\left(v(P(\mathbf{z}))^{N} P(\mathbf{z})\right) v(P(\mathbf{z}))=1$. Comparing this result and equation 2.15) we can deduce from the second item (only uniqueness is used) that

$$
\nu(P(\mathbf{z}))=\hat{v}(\mathbf{z}) .
$$

Hence $\tilde{v}_{i}(\mathbf{z})=\hat{v}(\mathbf{z})^{w_{i}}=v(P(\mathbf{z}))^{w_{i}}$, hence $A^{-1}$ is of the desired form as stated in part a) of the assertion. Note that $\hat{v}(0)=1 / u(0)$ by its definition and therefore also $v(0)=1 / u(0)$.

The proof of part a) is not yet quite complete. What about the uniqueness of $v$ when we have just given $u$ ? By its very definition, $\hat{v}$ is uniquely determined by $u$ (and $P$ ). Since $v(P(\mathbf{z}))=\hat{v}(\mathbf{z})$ and since $P$ is surjective onto a neighbourhood of zero, also $v$ is uniquely determined by $u$.

For part c) of the assertion we note that the operator $E$ which asigns to $u$ the function $v$ is given by solving the implicit equation $u\left(v^{N} t\right) v=1$ with $v(0)=1 / u(0)$. So let $E(u)=v$ and $E(v)=w$. Then we also have $v\left(w(s)^{N} s\right) w(s)=$ 1 for all $s \in(\mathbb{C}, 0)$. If in the equation $u\left(v(t)^{N} t\right) v(t)=1$ we substitute $t=$ $w(s)^{N} s$, we get

$$
\begin{aligned}
u\left[v\left(w(s)^{N} s\right)^{N} w(s)^{N} s\right] \nu\left(w^{N} s\right) & =1 \\
u\left[1^{N} s\right] \cdot 1 / w(s) & =1 \\
u(s) & =w(s) .
\end{aligned}
$$

This shows part c). 
It remains to prove part b). The $(i, j)$ th entry in the Jacobian matrix of the transformation

$$
A^{-1}: \quad \mathbf{z} \mapsto\left(\nu(P(\mathbf{z}))^{w_{1}} z_{1}, \ldots, v(P(\mathbf{z}))^{w_{n}} z_{n}\right)
$$

is given by

$$
\begin{aligned}
\partial_{i}\left(\nu(P(\mathbf{z}))^{w_{j}} z_{j}\right) & =w_{j}(\nu(P(\mathbf{z})))^{w_{j}-1} v^{\prime}(P(\mathbf{z})) \partial_{i} P(\mathbf{z}) z_{j}+v\left(P(\mathbf{z})^{w_{j}} \delta_{i j}\right. \\
& =(\nu(P(\mathbf{z})))^{w_{j}-1}\left[w_{j} v^{\prime}(P(\mathbf{z})) \partial_{i} P(\mathbf{z}) z_{j}+v(P(\mathbf{z})) \delta_{i j}\right]
\end{aligned}
$$

In order to compute its determinant we use lemma 2.19 from above. This together with the Euler relation for weighted homogeneous polynomials yields

$$
\begin{aligned}
& \operatorname{det}\left(D A^{-1}(\mathbf{z})\right) \\
& =\prod_{j=1}^{n}(\nu(P(\mathbf{z})))^{w_{j}-1} \cdot \operatorname{det}\left(\nu^{\prime}(P(\mathbf{z})) \partial_{i} P(\mathbf{z}) w_{j} z_{j}+v(P(\mathbf{z})) \delta_{i j}\right) \\
& =\prod_{j=1}^{n}(\nu(P(\mathbf{z})))^{w_{j}-1} \cdot(\nu(P(\mathbf{z})))^{n-1}\left[v(P(\mathbf{z}))+v^{\prime}(P(\mathbf{z})) \sum_{j=1}^{n} w_{j} z_{j} \partial_{j} P(\mathbf{z})\right] \\
& =v(P(\mathbf{z}))^{w_{1}+\ldots+w_{n}-n+n-1} \cdot\left[v(P(\mathbf{z}))+v^{\prime}(P(\mathbf{z})) N P(\mathbf{z})\right] \\
& =\left.\left(v(P)^{w}+\frac{N}{w} P \frac{d}{d P} v(P)^{w}\right)\right|_{P=P(\mathbf{z})},
\end{aligned}
$$

where we used the abbreviation $w=w_{1}+\ldots+w_{n}$.

Given $u$, we get the map $A$ of the lemma which we also denote by $A_{u}$. Then we have

$$
A_{E(u)} \circ A_{u}=\mathrm{id} .
$$

We make a remark which however will not be used elsewhere in this section. Assume that instead of $u$ we have just given the map $A$ (of the form $A_{u}$ with an unspecified $u$ ). Of course the $\tilde{v}_{i} z_{i}$ which are the component functions of $A^{-1}$ are uniquely determined by $A$. Then from $\tilde{v}_{i}(\mathbf{z})=\hat{v}(\mathbf{z})^{w_{i}}$ we infer that the function $\hat{v}$ is uniquely determined up to the multiplication 


\section{Milnor Number and Volume-Preserving Geometry}

with some number $\xi \in \mathbb{C}$ which fulfills $\xi^{w_{i}}=1$ for all $i$. If we demand that the greatest common divisor of the $w_{1}, \ldots, w_{n}$ is equal to one, then $\xi=1$ and so $\hat{v}$ and also $v$ are uniquely determined by $A$. Applying this argument to $A^{-1}$ we see that given a map $A$ (of the form $A_{u}$ with some unknown $u \in \operatorname{Units}\left(\mathscr{O}_{\mathbb{C}, 0}\right)$ ) the $u$ is uniquely determined if $\operatorname{gcd}\left(w_{1}, \ldots, w_{n}\right)=1$.

Let $\left(f_{0}, \Omega_{0}\right)$ be a pair consisting of a germ of a function $f_{0} \in \mathscr{O}_{\mathbb{C}^{n}, 0}$ which vanishes at the origin and a germ of a volume form $\Omega_{0} \in \Omega_{\mathbb{C}^{n}, 0}^{n}$. Then the group Aut $\left(\mathbb{C}^{n}, 0\right)$ acts on the set of such pairs by the usual pulling back of functions resp. forms. A normal form for a pair $\left(f_{0}, \Omega_{0}\right)$ should then be a nicely chosen pair in the same orbit. One way to achieve this is to look only at pairs in the orbit of $\left(f_{0}, \Omega_{0}\right)$ with the same $f=f_{0}$. Another way would be to consider only those pairs in the orbit of $\left(f_{0}, \Omega_{0}\right)$ with the same $\Omega=\Omega_{0}$. The latter would give us an $\Omega_{0}$-preserving normal form for functions which are right equivalent to $f_{0}$. That these two approaches are interchangeable when the right normal form is chosen is the content of the following lemma which we will later only use in the direction $(i i) \Rightarrow(i)$.

Lemma 2.22 (Exchange Lemma).

Let $P$ be quasihomogeneous of type $\left(w_{1}, \ldots, w_{n} ; N\right)$. For a holomorphic function germ $f=f(\mathbf{y}):\left(\mathbb{C}^{n}, 0\right) \rightarrow(\mathbb{C}, 0)$ the following statements are equivalent:

i) There exist an automorphism $\Phi \in \operatorname{Aut}\left(\mathbb{C}^{n}, 0\right), \mathbf{y} \mapsto \mathbf{x}$ and an automorphism $\Psi \in \operatorname{Aut}(\mathbb{C}, 0)$ such that

$$
f\left(\Phi^{-1}(\mathbf{x})\right)=\Psi(P(\mathbf{x})) \text { and }\left(\Phi^{-1}\right)^{*} d^{n} \mathbf{y}=d^{n} \mathbf{x} .
$$

ii) There exist an automorphism $\phi \in \operatorname{Aut}\left(\mathbb{C}^{n}, 0\right), \mathbf{z} \mapsto \mathbf{y}$ and a function $\psi \in$ $\mathscr{O}_{\mathbb{C}, 0}$ with $\psi(0) \neq 0$ such that

$$
f(\phi(\mathbf{z}))=P(\mathbf{z}) \text { and } \phi^{*} d^{n} \mathbf{y}=\psi(P(\mathbf{z})) d^{n} \mathbf{z} .
$$

Proof. We start with the implication $(i) \Rightarrow(i i)$. Since $\Psi^{\prime}(0) \neq 0$ there is a germ $u \in \mathscr{O}_{\mathbb{C}, 0}, u(0) \neq 0$ with $\Psi(t)=t u(t)^{N}$. From the quasihomogeneity of $P$ we get

$$
\begin{aligned}
\Psi(P(\mathbf{x})) & =P(\mathbf{x}) u(P(\mathbf{x}))^{N} \\
& =P\left(u(P(\mathbf{x}))^{w_{1}} x_{1}, \ldots, u(P(\mathbf{x}))^{w_{n}} x_{n}\right) .
\end{aligned}
$$


If we define the map

$$
A:\left(\mathbb{C}^{n}, 0\right) \rightarrow\left(\mathbb{C}^{n}, 0\right), \mathbf{x} \mapsto \mathbf{z}:=\left(u(P(\mathbf{x}))^{w_{1}} x_{1}, \ldots, u(P(\mathbf{x}))^{w_{n}} x_{n}\right)
$$

then $\Psi(P(\mathbf{x}))=P(A(\mathbf{x}))$. The first part of item $(i), f\left(\Phi^{-1}(\mathbf{x})\right)=\Psi(P(\mathbf{x}))$ can therefore be rewritten as $f\left(\Phi^{-1}(\mathbf{x})\right)=P(A(\mathbf{x}))$. Since by lemma 2.21 the map $A$ is an automorphism of $\left(\mathbb{C}^{n}, 0\right)$, we can rewrite this again: we let $\phi \in \operatorname{Aut}\left(\mathbb{C}^{n}, 0\right)$, $\mathbf{z} \mapsto \mathbf{y}$ with $\phi:=\Phi^{-1} \circ A^{-1}$, then it follows $f(\phi(\mathbf{z}))=P(\mathbf{z})$. This is the first assertion of item ( $i$ i ).

Again by lemma 2.21 there is $v \in \mathscr{O}_{\mathbb{C}, 0}, v(0) \neq 0$ with

$$
\operatorname{det}\left(D A^{-1}(\mathbf{z})\right)=\left.\left(v(P)^{w}+\frac{N}{w} P \frac{d}{d P} v(P)^{w}\right)\right|_{P=P(\mathbf{z})} .
$$

If we define $\psi:(\mathbb{C}, 0) \rightarrow \mathbb{C}$ by this bracket, i.e.

$$
\psi(t):=v(t)^{w}+\frac{N}{w} t \frac{d}{d t}\left(v(t)^{w}\right),
$$

then $\psi(0) \neq 0$ and we can write the pullback of the volume form as

$$
\begin{aligned}
\phi^{*} d^{n} \mathbf{y} & =\left(A^{-1}\right)^{*}\left(\Phi^{-1}\right)^{*} d^{n} \mathbf{y} \\
& =\left(A^{-1}\right)^{*} d^{n} \mathbf{x} \\
& =\psi(P(\mathbf{z})) d^{n} \mathbf{z} .
\end{aligned}
$$

This is the second assertion of item ( $i i)$.

Now we prove the converse direction. So let us assume $(i i)$ is valid. First we seek a solution $v:(\mathbb{C}, 0) \rightarrow \mathbb{C}, v(0) \neq 0$ of the equation

$$
\left(v(t)^{w}+\frac{N}{w} t \frac{d}{d t} v(t)^{w}\right)=\psi(t)
$$

where $\psi$ is the function as given in statement (ii), i.e. $\psi:(\mathbb{C}, 0) \rightarrow \mathbb{C}, \psi(0) \neq$ 0 . A solution can be obtained from a power series ansatz, namely if $\psi(t)=$ $\sum a_{i} t^{i}$ and $v^{w}=\sum b_{i} t^{i}$, then comparison of the coefficients shows that the stipulation

$$
b_{i}:=\frac{a_{i}}{1+(N i / w)}
$$




\section{Milnor Number and Volume-Preserving Geometry}

will provide a solution $v^{w}$ of the differential equation. Since $\psi(0)$ is nonzero so is $v^{w}(0)$. Hence, taking some $w$ th root $v$ of $v^{w}$ will give us $v$.

Now we define $u \in \mathscr{O}_{\mathbb{C}, 0}$ as $u=E^{-1}(v)$, cf. lemma 2.21. Then $\operatorname{det}\left(D A_{u}^{-1}(\mathbf{z})\right)=$ $\operatorname{det}\left(D A_{\nu}(\mathbf{z})\right)=\psi(P(\mathbf{z}))$ by that lemma and the definition of $v$. Now define $\Phi:=A_{u}^{-1} \phi^{-1}$. Then

$$
\begin{aligned}
\left(\Phi^{-1}\right)^{*} d^{n} \mathbf{y} & =\left(\phi \circ A_{u}\right)^{*} d^{n} \mathbf{y} \\
& =A_{u}^{*} \phi^{*} d^{n} \mathbf{y} \\
& =A_{u}^{*}\left(\psi(P(\mathbf{z})) d^{n} \mathbf{z}\right) \\
& =\psi\left(P\left(A_{u}(\mathbf{x})\right)\right) \operatorname{det} D A_{u}(\mathbf{x}) d^{n} \mathbf{x} \\
& =\psi(P(\mathbf{z})) \operatorname{det} D A_{u}(\mathbf{x}) d^{n} \mathbf{x} \\
& =d^{n} \mathbf{x}
\end{aligned}
$$

Finally when we insert into the given relation $f(\phi(\mathbf{z}))=P(\mathbf{z})$ the expres$\operatorname{sion} \mathbf{z}=A_{u}(\mathbf{x})$ we can rewrite it as

$$
\begin{aligned}
f\left(\Phi^{-1}(\mathbf{x})\right) & =P\left(A_{u}(\mathbf{x})\right) \\
& =P\left(u(P(\mathbf{x}))^{w_{1}} x_{1}, \ldots, u(P(\mathbf{x}))^{w_{n}} x_{n}\right) \\
& =P(\mathbf{x}) u(P(\mathbf{x}))^{N} .
\end{aligned}
$$

So letting $\Psi(t):=t u(t)^{N}$ we have the statement $f \circ \Phi^{-1}(\mathbf{x})=\Psi(P(\mathbf{x}))$ of our assertion. We note $\Psi^{\prime}(0)=u(0)^{N}=1 / v(0)^{N} \neq 0$, so $\Psi$ is an automorphism of $(\mathbb{C}, 0)$. This completes the proof.

We now show that part $(i i)$ in lemma 2.22 is true for $P=x_{1} \cdots x_{n}$.

Now let $f$ be right equivalent to this $P$. Choose $\Phi_{1} \in \operatorname{Aut}\left(\mathbb{C}^{n}, 0\right)$ with $\Phi_{1}^{*} f=$ $P$. Using proposition 2.4 we see that there exists $\psi \in \mathbb{C}\{t\}$ and $\eta$ with $d P \wedge \eta=$ $0, \eta(0)=0$ such that $\Phi_{1}^{*} d^{n} \mathbf{x}=\psi \circ P d^{n} \mathbf{x}+d \eta$. Now it is important to note - as shown in the proof by Françoise - that among the power series terms on the left-hand side only the constant term, i.e. $\operatorname{det}\left(D \Phi_{1}\right)(0)$, will contribute to the constant term of $\psi$ and they are equal. In particular $\psi(0) \neq 0$.

We now make use of the 
Lemma 2.23. Let $g \in \mathfrak{m}_{\mathbb{C}^{n}, 0}$ and $\Omega_{1}, \Omega_{2}$ two $n$-forms on $\left(\mathbb{C}^{n}, 0\right)$ with the same nonzero value at the origin. If there is an $(n-1)$-form $\eta, \eta(0)=0$ with $\Omega_{1}-$ $\Omega_{2}=d \eta$ such that $d g \wedge \eta=0$, then there exists $\Phi_{2} \in \operatorname{Aut}\left(\mathbb{C}^{n}, 0\right)$ with $\Phi_{2}^{*} g=g$ and $\Phi_{2}^{*} \Omega_{1}=\Omega_{2}$.

The proof is based on the path method and can be found in Fra82.

Applying it to $\Omega_{1}:=\Phi_{1}^{*} d^{n} \mathbf{x}, \Omega_{2}:=\psi \circ P d^{n} \mathbf{x}$ and $g:=P$ we get an automorphism $\Phi_{2}$ with $\Phi_{2}^{*} \Phi_{1}^{*} d^{n} \mathbf{x}=\psi \circ P d^{n} \mathbf{x}$ and $\Phi_{2}^{*} P=P$. So if we put $\phi:=\Phi_{1} \circ \Phi_{2}$ we have

$$
\phi^{*} d^{n} \mathbf{x}=\psi \circ P d^{n} \mathbf{x} \quad \text { and } \quad \phi^{*} f=P .
$$

This is item (ii) of lemma 2.22 . The implication $(i i) \Rightarrow(i)$ thus yields the existence of the normal form.

We now address the question of unicity of $\Psi$. The equation $f \circ \Phi(y)=$ $\Psi(P(y))$ can be written as a commutative diagram

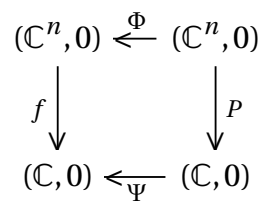

For sufficiently small $\epsilon>0$ and for all sufficiently small $0<\delta \ll \epsilon$ we have the Milnor-Lê fibration $f: B_{\epsilon} \cap f^{-1}\left(D_{\delta}^{*}\right) \rightarrow D_{\delta}^{*}$ where $B_{\epsilon}$ is the open $\epsilon$-ball around $0 \in \mathbb{C}^{n}$ and $D_{\delta}^{*}$ is the open $\delta$-ball around the origin in $\mathbb{C}$ minus this point. The general fibre is called the Milnor fibre $\operatorname{Mil}_{f, 0}$ of $f$. For a quasihomogeneous $P$ we can compute the Milnor fibre as the general fibre of the global affine fibration $P: \mathbb{C}^{n} \backslash P^{-1}(0) \rightarrow \mathbb{C}^{*}$, see ([Dim92], p. 68 - 72). Hence the Milnor fibre of $P$ over $s \in D_{\delta}^{*}$

$$
\operatorname{Mil}_{P, 0}(s)=\left\{\mathbf{x} \in B_{\epsilon} \mid x_{1} \cdots x_{n}=s\right\}
$$

is diffeomorphic to

$$
\left\{\left(x_{1}, \ldots, x_{n}\right) \in \mathbb{C}^{n} \mid x_{1} \cdots x_{n}=1\right\} \cong\left\{\left(x_{2}, \ldots, x_{n}\right) \in\left(\mathbb{C}^{*}\right)^{n-1}\right\} .
$$

Similar statements hold if replace the standard ball $B_{\epsilon}$ by a ball defined by a rug function $\left(B_{\epsilon}(\rho)=\left\{\mathbf{x} \in \mathbb{C}^{n} \mid \rho(\mathbf{x})<\epsilon\right\}\right.$ where $\rho:\left(\mathbb{C}^{n}, 0\right) \rightarrow \mathbb{R}_{\geq 0}$ is real analytic 
such that $\rho^{-1}(0)=\{0\}$.) So $H_{n-1}\left(\operatorname{Mil}_{P, 0}(s) ; \mathbb{Z}\right) \cong \mathbb{Z}$ with generator $\gamma(P, s)$ given by the product of $(n-1)$ circles. In fact for $s$ real and $s<(\epsilon / \sqrt{n})^{n}$ we have a map

$$
\underbrace{S^{1} \times \ldots \times S^{1}}_{n-1} \hookrightarrow \operatorname{Mil}_{P, 0}(s),\left(z_{2}, \ldots, z_{n}\right) \mapsto\left(s^{1 / n} /\left(z_{2} \ldots z_{n}\right), z_{2} s^{1 / n}, \ldots, z_{n} s^{1 / n}\right),
$$

which is easily checked to be an embedding. Along this cycle we can integrate any holomorphic $(n-1)$-form $\lambda$ and if we choose $\lambda$ as a holomorphic primitive of $d^{n} \mathbf{x}$, e.g. $\lambda=x_{1} d x_{2} \wedge \ldots \wedge d x_{n}$, then we evaluate the integral of $\lambda$ over one of the generators of $H_{n-1}\left(\operatorname{Mil}_{P, 0}(s) ; \mathbb{Z}\right)$ as

$$
\int_{\gamma(P, s)} \lambda=\int_{S^{1} \times \ldots \times S^{1}} s \frac{d z_{2}}{z_{2}} \ldots \frac{d z_{n}}{z_{n}}= \pm(2 \pi i)^{n-1} s .
$$

(Of course, if we had chosen the canonical orientation of $\operatorname{Mil}_{P, 0}(s)$ as a complex manifold we would get a plus sign, but it is not important here.)

Finally let $\gamma(f, \cdot)$ be a locally constant section of the $(n-1)$ st homological fibration of $f$, obtained by parallel translating one of the two homology generators of a single reference fibre. Then we get an a priori multivalued holomorphic function germ $t \mapsto \int_{\gamma(f, t)} \lambda$.

From the commutativity of the above diagram it follows that an integral homology generator of $\operatorname{Mil}_{P, 0}\left(\Psi^{-1}(t)\right)$ is sent via $\Phi_{*}$ to one of the two generators of $H_{n-1}\left(\operatorname{Mil}_{f, 0}(t) ; \mathbb{Z}\right)$ and so we obtain

$$
\int_{\gamma(f, t)} \lambda= \pm \int_{\Phi_{*} \gamma\left(P, \Psi^{-1}(t)\right)} \lambda .
$$

Now $\Phi$ being volume-preserving, it preserves $\lambda$ up to a differential, so the right-hand side becomes

$$
\begin{aligned}
& \int_{\gamma\left(P, \Psi^{-1}(t)\right)} \Phi^{*} \lambda \\
& =\int_{\gamma\left(P, \Psi^{-1}(t)\right)} \lambda \\
& = \pm(2 \pi i)^{n-1} \Psi^{-1}(t) .
\end{aligned}
$$

Hence $\Psi^{-1}(t)= \pm\left(\frac{1}{2 \pi i}\right)^{n-1} \int_{\gamma(f, t)} \lambda$ so that $\Psi$ is uniquely determined by $f$, 
possibly up to a sign.

And indeed we show that the alleged ambiguity in the choice of $\Psi$ 's sign cannot be eliminated: Take any permutation matrix $S \in \mathbb{C}^{n \times n}$ with determinant -1 and let $c$ be any number with $c^{n}=-1$. Then the linear map $\mathbf{x} \mapsto$ $\Phi(\mathbf{x}):=c S \mathbf{x}$ is volume-preserving and transforms $x_{1} \cdots x_{n}$ to $\left(c x_{1}\right) \cdots\left(c x_{n}\right)=$ $-x_{1} \cdots x_{n}$. 


\section{Milnor Number and Meromorphic Germs}

\subsection{Pencils and Bifurcation}

Throughout this section we assume that $f, g \in \mathbb{C}\{x, y\}$ vanish at the origin and are coprime. In this situation we may assign to the pair $(f, g)$ a finite set $\mathscr{B}(f, g)$ which is a subset of $\mathbb{P}^{1}$ such that roughly spoken the pencil members $s f+t g$ where $(s: t) \in \mathscr{B}(f, g)$ have different geometry than the remaining members. We give the following different but equivalent definitions of this bifurcation set:

- $\mathscr{B}_{j p}$ : the bifurcation set determined by the jumping of the Milnor number,

- $\mathscr{B}_{\text {alg }}$ : the bifurcation set from the algorithm by Maugendre and Michel,

- $\mathscr{B}_{\text {disc }}$ : the bifurcation set as a projective tangent cone of the discriminant,

- $\mathscr{B}_{\text {top }}$ : the bifurcation set determined by the embedded topological type

There are other important characterizations which are quite difficult to relate. For example one can look at minimal resolutions of the meromorphic germ $(f: g):\left(\mathbb{C}^{2}, 0\right) \rightarrow \mathbb{P}^{1}$, compare [LW97]. One can also look at gradientlike descriptions of $\mathscr{B}$. They are based on Milnor's proof of the knottheoretic Milnor fibration as he introduced an analytic condition to see whether the fibres of the holomorphic map $f$ are not transverse to spheres around the origin. This has been mimicked for meromorphic germs by Pichon [BP07] and was used when they proved that there exists a knottheoretic Milnor fibration

$$
\frac{f / g}{|f / g|}: S_{\epsilon}^{3} \backslash V(f g) \rightarrow S^{1}
$$


under the assumption that $f, g \in \mathfrak{m}_{\mathbb{C}^{2}, 0}$ are coprime with bifurcation set contained in $\{0, \infty\}$. They also investigated the relation with other fibration theorems for meromorphic germs. However we shall not go deeper into this direction.

It seems appropriate to start our investigation with the following theorem which to my knowledge is essentially due to Zariski ([Zar65a], [Zar65b]) and has been extended by Teissier and others to further equivalent conditions ([Tei76], p. 623) dealing e.g. with the resolutions of singularities.

Theorem 3.1. (Equisingularity of Plane Curves)

Let $f:(X, 0) \rightarrow(Y, 0)$ be a flat family of reduced plane curve germs parametrized by a smooth complex space germ $(Y, 0)$ and let $\sigma:(Y, 0) \rightarrow(X, 0)$ be a holomorphic section. Denote by $r, \delta$, mult the number of irreducible components, the $\delta$-invariant resp. the multiplicity of the singularity. Then the following conditions are equivalent:

a) The map $y \mapsto \mu\left(X_{y}, \sigma(y)\right)$ is constant.

b) The maps $y \mapsto \delta\left(X_{y}, \sigma(y)\right)$ and $y \mapsto r\left(X_{y}, \sigma(y)\right)$ are constant.

c) The maps $y \mapsto \mu\left(X_{y}, \sigma(y)\right)$ and $y \mapsto m\left(X_{y}, \sigma(y)\right)$ are constant.

It might be useful to recall at that point Milnor's formula $\mu(f)=2 \delta(f)-$ $r(f)+1$ for an isolated (plane) singularity $f \in \mathfrak{m}_{\mathbb{C}^{2}, 0}$.

Now let us fix some $\left(s_{0}: t_{0}\right) \in \mathbb{P}^{1}$ and investigate the space $X$ as a germ at $\{(0,0)\} \times\left\{\left(s_{0}: t_{0}\right)\right\}$ defined by the vanishing of $s f+t g$ in $\left(\mathbb{C}^{3},\left((0,0),\left(s_{0}: t_{0}\right)\right)\right)$. Let us denote by $\pi: \mathbb{C}^{3} \rightarrow \mathbb{C}$ the map which sends $(x, y, \lambda)$ to $\lambda$. By choosing suitable coordinates for $\mathbb{P}^{1}$ (i.e. $\lambda=s / t$ or $\lambda=t / s$ ) we can restrict $\pi$ to $X$ and call the associated germ of a mapping $\pi:\left(X,\{(0,0)\} \times\left\{\left(s_{0}: t_{0}\right)\right\}\right) \rightarrow\left(\mathbb{C}, \lambda_{0}\right)$. Then one can see that the singular locus of $X$ is contained in $\{(0,0)\} \times \mathbb{P}^{1}$. In the terminology of e.g. [BGG80] we have

Proposition 3.2. The map germ $\pi:\left(X,\{\mathbf{0}\} \times\left\{\lambda_{0}\right\}\right) \rightarrow\left(\mathbb{C}, \lambda_{0}\right)$ is a flat and centered deformation of its central fibre.

Proof. For a (finitely generated) Cohen-Macaulay module $M$ over a local Noetherian ring $(A, \mathfrak{m})$ and for elements $a_{1}, \ldots, a_{k} \in \mathfrak{m}$ it is known that $\operatorname{dim}(M /<$ 


\section{Milnor Number and Meromorphic Germs}

$\left.a_{1}, \ldots, a_{k}>M\right) \geq \operatorname{dim}(M)-k$ with equality if and only if $\left(a_{1}, \ldots, a_{k}\right)$ is an $M$ regular sequence. As hypersurface rings are always Cohen-Macaulay, we can apply this to $M=\mathscr{O}_{X}=\mathscr{O}_{\mathbb{C}^{3}, 0} / I$ where $I$ is the ideal generated by $f(x, y)+$ $\lambda g(x, y)$. Since $X$ has dimension two and the local ring associated to the zero fibre $\mathscr{O}_{X_{0}}=\mathscr{O}_{X} / \lambda \mathscr{O}_{X}$ has dimension one, we obtain that $(\lambda)$ is a $\mathscr{O}_{X}$-regular sequence, i.e. is a nonzero divisor. Hence $\mathscr{O}_{X}$ is a flat $\mathscr{O}_{\lambda}$-module.

One speaks of a centered deformation when the singular locus of $X$ has underlying space $\{\mathbf{0}\} \times(\mathbb{C}, 0)$.

We won't use this but it is helpful to put our situation in a broader context. Regarding the multiplicity we can even be more explicit than in the first theorem (on equisingularitiy):

Proposition 3.3. Let $f, g \in \mathfrak{m}_{\mathbb{C}^{2}, 0}$. Then all the pencil members $s f+\operatorname{tg},(s: t) \in$ $\mathbb{P}^{1}$, except maybe one, have the same multiplicity at the origin. More precisely we have

- If mult $(f) \neq$ mult $(g)$, a multiplicity jump occurs of course.

- If mult $(f)=\operatorname{mult}(g)$, a multiplicity jump occurs if and only if the leading parts of $f$ and $g$ are linearly dependent if and only if $f$ and $g$ have the same tangents including their multiplicities.

Proof. If $m(f)<m(g)$, then $m(s f+t g)=m(f)$ for all $(s: t) \neq(0: 1)$. So let us now come to the more interesting case $m(f)=m(g)$. We may then safely assume that $f$ and $g$ are homogeneous polynomials of degree $m$. Write $f(x, y)=\sum_{i=0}^{m} a_{i} x^{i} y^{m-i}$ and $g(x, y)=\sum_{i=0}^{m} b_{i} x^{i} y^{m-i}$. Then

$$
s f+t g=\sum_{i=0}^{m}\left(s a_{i}+t b_{i}\right) x^{i} y^{m-i} .
$$

The multiplicity of $s f+t g$ jumps to a higher value at this particular $(s: t)$ if and only if the above polynomial vanishes, i.e. $s a_{i}+t b_{i}=0$ for all $i=0, \ldots, m$. For such an $(s: t)$ we have $(s: t)=\left(-b_{i}: a_{i}\right)$ for all $i$ for which $a_{i}$ and $b_{i}$ are not both zero. Of course one such $i$ must exist, for otherwise $f$ and $g$ would be zero. Hence there occurs at most one jumping for the multiplicity and the rest of the assertion is immediate.

For the understanding of the bifurcation set the following formula is inevitable, since it relates the Milnor number of the pencil members to the 
critical locus of the map $(f, g)$. As Lê Dũng Tráng was so friendly to tell me, he found this formula in his $\mathrm{PhD}$ thesis (1969) and it was published in Vietnam in 1971. One year later he gave a talk in Göttingen about it, the time where Brieskorn had been (t)here. There is also a more general statement for complete intersections which was proved using Morse theory by Lê ([Lê74]) or using sheaf theory etc. by Greuel ([Gre75]). Nowadays there is a simpler proof, compare ([CA00, chapter 7).

Proposition 3.4 (Lê).

For $f, g:\left(\mathbb{C}^{2}, 0\right) \rightarrow(\mathbb{C}, 0)$ we have the following equality

$$
i\left(f, f_{x} g_{y}-f_{y} g_{x}\right)=\mu(f)+i(f, g)-1 .
$$

In case one of the two sides of this equation is infinite, so is the other. For this special case the author found a new proof which is presented below. We start with the following well-known lemma - a generalization to higher dimensions can be found in ([Loo84], Theorem (2.8.iii)).

Lemma 3.5. Assume that $a, b \in \mathbb{C}\{x, y\}$ vanish at the origin and are coprime. Then $a_{x} b_{y}-a_{y} b_{x}$ is not identically zero.

Proof. Here is a proof when $a b$ is reduced. Since $a$ defines an isolated singularity, $a_{x}$ and $a_{y}$ are coprime. Thus, there is a power series germ $c$ with $b_{x}=c a_{x}$ and $b_{y}=c a_{y}$. Now we compute

$$
\begin{aligned}
& (a b)_{x}=a_{x} b+a b_{x}=a_{x} b+a c a_{x}=a_{x}(b+c a), \\
& (a b)_{y}=a_{y} b+a b_{y}=a_{y} b+a c a_{y}=a_{y}(b+c a) .
\end{aligned}
$$

Since $a b$ defines an isolated singularity, $b+c a$ must be a unit in $\mathbb{C}\{x, y\}$, which is impossible since $a$ and $b$ vanish at the origin.

In general, i.e. when $a$ or $b$ is not neccessarily reduced, we observe that since $(a, b)=1$ and $a(0)=b(0)=0$, the map $(a, b):\left(\mathbb{C}^{2}, 0\right) \rightarrow\left(\mathbb{C}^{2}, 0\right)$ is surjective. Now $V\left(a_{x} b_{y}-a_{y} b_{x}\right)$ is the critical set of $(a, b)$ and if it was the whole $\left(\mathbb{C}^{2}, 0\right)$, then $(a, b)$ would have no regular values at all, contradicting Sard's theorem.

So $V\left(a_{x} b_{y}-a_{y} b_{x}\right) \subset\left(\mathbb{C}^{2}, 0\right)$ is either a curve germ (if $a_{x}(0) b_{y}(0)-a_{y}(0) b_{x}(0)=$ 0 ) or void (if $a_{x}(0) b_{y}(0)-a_{y}(0) b_{x}(0) \neq 0$ ). The latter happens if and only if $(f, g)$ is right equivalent to $(x, y)$ (cf. the proof of proposition 3.10). 


\section{Milnor Number and Meromorphic Germs}

Theorem 3.6. Let $f, g \in \mathbb{C}\{x, y\}$ both vanish at the origin. Then the following statements are equivalent:

1. the germ $f$ is reduced and $f, g$ are coprime,

2. the germs $f$ and $\{f, g\}=f_{x} g_{y}-f_{y} g_{x}$ are coprime.

Proof. The direction (2) $\Rightarrow(1)$ is easily proved by contraposition: If $f$ was nonreduced, say $f=p^{m} q$ for some $p, q \in \mathbb{C}\{x, y\}$ with $p(0)=0$ and $m \geq 2$, then we compute

$$
\begin{aligned}
f_{x} g_{y}-f_{y} g_{x} & =\left(m p^{m-1} p_{x} q+p^{m} q_{x}\right) g_{y}-\left(m p^{m-1} p_{y} q+p^{m} q_{y}\right) g_{x} \\
& =p\left[\left(m p^{m-2} p_{x} q+p^{m-1} q_{x}\right) g_{y}-\left(m p^{m-2} p_{y} q+p^{m-1} q_{y}\right) g_{x}\right],
\end{aligned}
$$

and hence the non-unit $p$ divides $f$ and $D(f, g):=\{f, g\}$.

Now for the converse implication. So $f$ is reduced and we can write $f=$ $f_{1} \ldots f_{r}$ where each $f_{i}$ is irreducible and the $f_{i}$ 's are pairwise coprime. Since $\{f, g\}$ is a derivation in each entry we may even assume that $f$ is $f$ is irreducible. Now if $f$ and $\{f, g\}$ would not be coprime, then the irreducible $f$ would divide $D$, hence there would be $k \in \mathbb{C}\{x, y\}$ with $f k=f_{x} g_{y}-f_{y} g_{x}$.

Since $f$ has an isolated singularity at the origin, the Brieskorn module $H:=\frac{\Omega^{2}}{\{d f \wedge d h \mid h \in \mathbb{C}\{x, y\}\}}$ is a free $\mathbb{C}\{f\}$-module of finite rank. In particular it is torsionfree, i.e. multiplication with $f$ is injective. Let $\omega_{0}=d x \wedge d y$ be the standard volume form. Then $f k \omega_{0}$ is zero in $H$, hence by what was just said, $k \omega_{0}$ is zero in $H$. This means that there exists $h \in \mathbb{C}\{x, y\}$ with $k=f_{x} h_{y}-f_{y} h_{x}$. Together with the definition of $k$ we get $f\left(f_{x} h_{y}-f_{y} h_{x}\right)=f_{x} g_{y}-f_{y} g_{x}$, or by rearranging the terms $f_{x}\left(g_{y}-f h_{y}\right)-f_{y}\left(g_{x}-f h_{x}\right)=0$, which is easily checked to be equivalent to

$$
f_{x}(g-f h)_{y}-f_{y}(g-f h)_{x}=0 .
$$

The greatest common divisor of $f$ and $g-f h$ is again a unit and these functions vanish at the origin. To finish the proof, we just apply the previous lemma.

After these preparations we give several definitions of the bifurcation set. We assume that $f, g:\left(\mathbb{C}^{2}, 0\right) \rightarrow(\mathbb{C}, 0)$ are given coprime germs. There is a uniquely determined set $\mathscr{B}_{\text {top }}(f, g) \subset \mathbb{P}^{1}$ such that the following properties hold for all $\left(s_{1}: t_{1}\right),\left(s_{2}: t_{2}\right) \in \mathbb{P}^{1}$ : 
a) $\mathscr{B}(f, g)_{\text {top }}$ is a finite set

b) if $s_{1} f+t_{1} g$ has a nonisolated singularity at the origin, then $\left(s_{1}: t_{1}\right) \in$ $\mathscr{B}(f, g)_{\text {top }}$,

c) if $s_{1} f+t_{1} g$ and $s_{2} f+t_{2} g$ both have an isolated singularity at the origin, then

i) $\left(\{0\}, V\left(s_{1} f+t_{1} g\right), \mathbb{C}^{2}\right) \approx\left(\{0\}, V\left(s_{2} f+t_{2} g\right), \mathbb{C}^{2}\right)$ if $\left(s_{1}: t_{1}\right),\left(s_{2}: t_{2}\right)$ are both not in $\mathscr{B}(f, g)_{\text {top }}$,

ii) $\left(\{0\}, V\left(s_{1} f+t_{1} g\right), \mathbb{C}^{2}\right) \not \approx\left(\{0\}, V\left(s_{2} f+t_{2} g\right), \mathbb{C}^{2}\right)$ if $\left(s_{1}: t_{1}\right) \in \mathscr{B}(f, g)_{\text {top }}$ but $\left(s_{2}: t_{2}\right) \notin \mathscr{B}(f, g)_{\text {top }}$

Above we have used $\approx$ as an abbreviation for homeomorphism of triples of germs of sets at the origin. By definition of $\mathscr{B}_{t o p}(f, g)$ the pencil members $s f+t g$ with $(s: t) \notin \mathscr{B}_{t o p}(f, g)$ have the same Milnor number. (Recall here that the Milnor number is a topological invariant.) This common Milnor number will be denoted by $\mu_{g e n}(f, g)$.

An alternative descrition of the bifurcation set is given by

$$
\mathscr{B}_{j p}(f, g)=\left\{(s: t) \in \mathbb{P}^{1} \mid \infty \geq \mu(s f+t g)>\mu_{g e n}(f, g) \equiv \min _{(\alpha: \beta) \in \mathbb{P}^{1}} \mu(\alpha f+\beta g)\right\} .
$$

Proposition 3.7. $\mathscr{B}_{\text {top }}(f, g)=\mathscr{B}_{j p}(f, g)$

Proof. This follows from the famous result of Lê and Ramanujam ([LR76]).

The next proposition gives another characterization of the bifurcation set. Let $C=\{f, g\}$ and $C=\prod C_{i}^{a_{i}}$ its prime factor decomposition. Since $\Phi:=$ $(f, g):\left(\mathbb{C}^{2}, 0\right) \rightarrow\left(\mathbb{C}^{2}, 0\right)$ is a finite map, the image of $C_{i}$ under $\Phi$ is a curve germ $\Delta_{i}$ and we let $d_{i}$ be the degree of the branched covering $C_{i} \rightarrow \Delta_{i}$. Finally let $\Delta=\Phi(C)$. The first part of the following formula is well-known (see e.g. [Pł04], the second should be known but I know of no reference. Płoski has given also another formula for $\mu_{\text {gen }}$.

Proposition 3.8. Let $f, g \in \mathfrak{m}_{\mathbb{C}^{2}, 0}$ be coprime. Then

1. $\mathscr{B}(f, g)$ is the projective tangent cone of $\Delta(f, g))$ and

2. $\mu_{g e n}(f, g)=1-i(f, g)+\sum a_{i} d_{i} \operatorname{mult}\left(\Delta(f, g)_{i}\right)$. 


\section{Milnor Number and Meromorphic Germs}

Proof. Let $U$ be a neighbourhood of the origin in $\mathbb{C}^{2}$ where $f$ and $g$ are defined and where the map $\Phi=(f, g): U \rightarrow V$ is finite. Let the coordinates in the image space be $(u, v)$.

According to the projection formula for the intersection multiplicity we have

$$
\begin{aligned}
i(f+a g,\{f, g\}) & =i\left(\Phi^{-1}(u+a v),\{f, g\}\right) \\
& =i\left(u+a v, \Phi_{*}\{f, g\}\right) \\
& =\sum_{k} a_{k} d_{k} i\left(u+a v, \Delta_{k}\right) .
\end{aligned}
$$

Appling the Lê'S formula to $f+a g$ instead of $f$ for any $a \in \mathbb{C}$, then $1-\mu(f+$ $a g)=i(f, g)-i(f+a g, \Gamma(f, g))$ shows that $\mu(f+a g)$ is minimal if and only if $i(f+a g,\{f, g\})$ becomes minimal.

It is well-known that for plane curves $C, D$ we have $i(C, D)=m(C) m(D)+$ $\sum_{p} i_{p}(\widehat{C}, \widehat{D})$ where the summation is over points in the exceptional divisor of the blowup of $\left(\mathbb{C}^{2}, 0\right)$ and $\widehat{C}, \widehat{D}$ are the respective strict transforms. Now it is also known that $\widehat{C} \cap \mathbb{E}$ consists of the points that corresponds to tangents of $C$. Hence $i(C, D)$ is minimal if and only if $C, D$ have no common tangents and the minimal value is the product of the multiplicities of $C, D$. In our situation this amounts to say that each $i\left(u+a v, \Delta_{i}\right)$ is minimal if and only if $a$ does not belong to the tangent cone of $\Delta_{i}$ and the minimal value of $\mu(f+a g)$ is $1-i(f, g)+\sum a_{i} d_{i} \operatorname{mult}\left(\Delta_{i}\right)$.

\section{Example.}

Let $f(x, y)=x^{m}+y^{n}$ and $g(x, y):=x^{p}+y^{q}$ with $m>p$ and $q \geq n$. We like to compare $i(f, C)$ with $i\left(u, \Phi_{*} C\right)$. (It should be the same.)

The analytic set germ

$$
C=f_{x} g_{y}-f_{y} g_{x}=x^{p-1} y^{n-1}\left(m q x^{m-p} y^{q-n}-p n\right)
$$

has two components and its associated cycle is $[C]=(p-1)[x]+(n-1)[y]$. We have $i(f, C)=(p-1) i\left(x^{m}+y^{n}, x\right)+(n-1) i\left(x^{m}+y^{n}, y\right)=(p-1) n+(n-1) m$. The component $C_{1}=V(x)$ is mapped under $\Phi$ to $\Delta_{1}=\left\{(u, v)=t^{n}, t^{q}\right) \mid t \in$ $(\mathbb{C}, 0)\}=V\left(u^{q / d}-v^{n / d}\right)$ where $d:=\operatorname{gcd}(n, q)$. Similarly the component $C_{2}=$ $V(y)$ is mapped under $\Phi$ to $\Delta_{2}=\left\{(u, v)=\left(t^{m}, t^{p}\right) \mid t \in(\mathbb{C}, 0)\right\}=V\left(u^{p / e}-v^{m / e}\right)$ where $e:=\operatorname{gcd}(m, p)$. The map $C_{1} \rightarrow \Delta_{1}$ is the map $\left(x^{m}+y^{n}, x^{p}+y^{q}\right): V(x) \rightarrow$ $V\left(u^{q / d}-v^{n / d}\right)$, the fibre over a general point, say $\left(t^{n}, t^{q}\right)$ for any $t \in\left(\mathbb{C}^{*}, 0\right)$, 
is given by $\left\{(0, y) \mid\left(y^{n}, y^{q}\right)=\left(t^{n}, t^{q}\right)\right\}$ which has cardinality $\operatorname{gcd}(n, q)=d$. In the same way one computes $\operatorname{deg}\left(C_{2} \rightarrow \Delta_{2}\right)=e$. Altogether we obtain

$$
\begin{aligned}
i\left(u, \Phi_{*} C\right) & =i\left(u,(p-1) \Phi_{*} C_{1}+(n-1) \Phi_{*}[y]\right) \\
& =i\left(u,(p-1) \operatorname{deg}\left(C_{1} \rightarrow \Delta_{1}\right)\left[\Delta_{1}\right]+(n-1) \operatorname{deg}\left(C_{2} \rightarrow \Delta_{2}\right)\left[\Delta_{2}\right]\right) \\
& =(p-1) \operatorname{di}\left(u,\left[\Delta_{1}\right]\right)+(n-1) \operatorname{ei}\left(u,\left[\Delta_{2}\right]\right) \\
& =(p-1) n+(n-1) m
\end{aligned}
$$

Using that $f+\lambda g=x^{m}+\lambda x^{p}+y^{n}+\lambda y^{q}$ is right equivalent to $x^{p}+y^{n}$ we see that the generic Milnor number of the pencil made up by $f$ and $g$ is $\mu_{\text {gen }}(f, g)=(p-1)(n-1)$.

On the other hand the generic Milnor number is by the formula : $\mu_{g e n}=$ $1-i(f, g)+\sum a_{i} d_{i} m\left(\Delta_{i}\right)=1-i(f, g)+(p-1) d(n / d)+(n-1) e(p / e)$. It can checked that $i(f, g)=p n$ : the germ $x^{m / c}+y^{n / c}$ with $c:=\operatorname{gcd}(m, n)$ is irreducible. That's why

$$
\begin{aligned}
i(f, g) & =c \cdot i\left(x^{m / c}+y^{n / c}, g\right) \\
& =c \cdot \operatorname{ord} g\left( \pm t^{n / c}, t^{m / c}\right) \\
& =c \cdot \operatorname{ord}\left( \pm t^{p n / c}+t^{m q / c}\right) \\
& =c \cdot(p n / c) \\
& =p n
\end{aligned}
$$

None of the previous definitions of the bifurcation set allows a computation of this finite set in concrete situations. This situation has been settled by the following algorithmic description of $\mathscr{B}(f, g)$ according to Maugendre and Michel ([MM01]):

Define a set $\mathscr{B}_{a l g}(f, g)$ as follows. Put $\mathscr{B}_{a l g}(f, g):=\varnothing$.

1. If $f$ and $g$ are both reduced, proceed as follows. If $\left(f_{x} g_{y}-f_{y} g_{x}\right)(0) \neq 0$ then $\mathscr{B}_{a l g}(f, g)=\varnothing$ and the algorithm stops. If $\left(f_{x} g_{y}-f_{y} g_{x}\right)(0)=0$, for each branch $\gamma$ of $f_{x} g_{y}-f_{y} g_{x}$ do the following.

- if $i(f, \gamma)>i(g, \gamma)$ add 0 to $\mathscr{B}_{a l g}(f, g)$,

- if $i(f, \gamma)<i(g, \gamma)$ add $\infty$ to $\mathscr{B}_{a l g}(f, g)$,

- if $i(f, \gamma)=i(g, \gamma)$, add $\lambda$ to $\mathscr{B}_{a l g}(f, g)$, where $\lambda \in \mathbb{C}^{*}$ is unique 


\section{Milnor Number and Meromorphic Germs}

number with $i(f+\lambda g, \gamma)>i(f, \gamma)$. Equivalently choose a parametrization $\phi(t)$ of $\gamma$ then (with $m:=i(f, \gamma)$ and $a \neq 0, b \neq 0$ ) we have

$$
\begin{aligned}
& f \circ \phi(t)=a t^{m}+\text { higher order terms } \\
& g \circ \phi(t)=b t^{m}+\text { higher order terms }
\end{aligned}
$$

and $\lambda$ is determined from the equation $a+b \lambda=0$.

2. If $f$ or $g$ is not reduced, apply an invertible linear transformation $A=$ $\left(\begin{array}{ll}a & b \\ c & d\end{array}\right)$ on the pair $(f, g)$ to get a pair $(a f+b g, c f+d g)$ with $a f+b g$ and $c f+d g$ both reduced. Proceed as in (1) to determine $\mathscr{B}_{a l g}(a f+$ $b g, c f+d g)$ and relate this to $\mathscr{B}_{a l g}(f, g)$.

Proposition 3.9. $\mathscr{B}_{\text {alg }}(f, g)_{\text {alg }}=\mathscr{B}_{j p}(f, g)$

Proof. We sketch the simpler proof by A. Płoski in [Pł04]. If $a \in \mathbb{P}^{1} \backslash\{\infty\}$ is such that $f_{a}$ is reduced, then theorem 2.2(i) in the cited article is the formula

$$
\mu(f+a g)-\mu_{g e n}=\sum_{i(f, \gamma) \geq i(g, \gamma)} \operatorname{ord}(\gamma)(i(f+a g, \gamma)-i(g, \gamma)),
$$

where the sum is over the different branches $\gamma$ of $f_{x} g_{y}-f_{y} g_{x}$ with the required property and ord $(\gamma)$ is the the multiplicity of $\gamma$ in the prime factor decomposition of $f_{x} g_{y}-f_{y} g_{x}$. From this formula the assertion can be read off, we make clear the following: if for some $a \in \mathbb{P}^{1} \backslash\{0, \infty\}$ we had $i(f+a g, \gamma)>$ $i(g, \gamma)$ then $i(f, \gamma)=i(g, \gamma)$. The values $a=\infty$ and values of $a$ for which $f_{a}$ is nonreduced are handled similarly. The deduction of the above equation makes use of Lê's formula.

Proposition 3.10. Let $f, g \in \mathfrak{m}_{\mathbb{C}^{2}, 0}$. Then $\mathscr{B}(f, g)$ is empty if and only if the pair $(f, g)$ is right-equivalent to $(x, y)$.

Proof. We have shown that the bifurcation set is the projective tangent cone of the discriminant. So $\mathscr{B}(f, g)$ is nonempty if and only if the discriminant is a curve. But this is always so except when $\left\{f_{x} g_{y}-f_{y} g_{x}=0\right\}$ is not the germ of a curve at $\left(\mathbb{C}^{2}, 0\right)$, i.e. when $C:=f_{x} g_{y}-f_{y} g_{x}$ is nonzero at the origin. Let us examine when this happens. If $f$ or $g$ is critical at the origin, then $C(0)=0$. So we can assume that $f$ and $g$ are smooth and after a suitable coordinate transformation we have $(f, g)=(x, a x+b y+o(x, y))$ in which case 
$C=b+\partial_{y} o(x, y)$. So $C(0) \neq 0$ if and only if $b \neq 0$. By a second coordinate transformation we have $(f, g) \sim(x, y)$.

\subsection{Milnor Number of Pairs of Functions}

In this section we introduce a generalization of the classical Milnor number, namely we associate to a pair of function germs a number $\mu(f, g)$ which I call Milnor number of pairs or meromorphic Milnor number.

For any $f, g \in \mathbb{C}\{x, y\}$ we introduce the notation $\langle\omega(f, g)\rangle$ for the ideal in $\mathbb{C}\{x, y\}$ generated by the elements $f_{x} g-f g_{x}$ and $f_{y} g-f g_{y}$. It is clear that $\langle\omega(f, g)\rangle$ is a subideal of $\langle f, g\rangle$. Sometimes if $f$ and $g$ are clear from the context, we simply write $\omega$ instead of $\omega(f, g)$. Let us now define the following possibly infinite numbers

$$
\begin{aligned}
\mu(f, g): & =\operatorname{dim}_{\mathbb{C}} \frac{\mathbb{C}\{x, y\}}{\langle\omega(f, g)\rangle}, \\
v(f, g): & =\operatorname{dim}_{\mathbb{C}} \frac{\langle f, g\rangle}{\langle\omega(f, g)\rangle} .
\end{aligned}
$$

Proposition 3.11. The numbers $\mu(f, g)$ and $v(f, g)$ just depend on the contact equivalence class of $(f, g)$. I.e. for any coordinate transformation $\Phi \in$ $\operatorname{Aut}\left(\mathbb{C}^{2}, \mathbf{0}\right)$ and any unit $u \in \mathbb{C}\{x, y\}$ we have $\mu(u \cdot f \circ \Phi, u \cdot g \circ \Phi)=\mu(f, g)$.

Proof. In fact by rearranging terms we get

$$
\begin{aligned}
\langle\omega(f \circ \Phi, g \circ \Phi)\rangle= & \left\langle\left(f_{x} \circ \Phi \Phi_{1 x}+f_{y} \circ \Phi \Phi_{2 x}\right) g \circ \Phi-\left(g_{x} \circ \Phi \Phi_{1 x}+g_{y} \circ \Phi \Phi_{2 x}\right) f \circ \Phi,\right. \\
& \left.\left(f_{x} \circ \Phi \Phi_{1 y}+f_{y} \circ \Phi \Phi_{2 y}\right) g \circ \Phi-\left(g_{x} \circ \Phi \Phi_{1 y}+g_{y} \circ \Phi \Phi_{2 y}\right) f \circ \Phi\right\rangle \\
= & \left\langle\Phi_{1 x}\left(f_{x} \circ \Phi g \circ \Phi-g_{x} \circ \Phi f \circ \Phi\right)+\Phi_{2 x}\left(f_{y} \circ \Phi g \circ \Phi-g_{y} \circ \Phi f \circ \Phi\right),\right. \\
& \left.\Phi_{1 y}\left(f_{x} \circ \Phi g \circ \Phi-g_{x} \circ \Phi f \circ \Phi\right)+\Phi_{2 y}\left(f_{y} \circ \Phi g \circ \Phi-g_{y} \circ \Phi f \circ \Phi\right)\right\rangle .
\end{aligned}
$$

Since $\operatorname{det} D \Phi(\mathbf{0}) \neq 0$ this yields

$$
\begin{aligned}
\langle\omega(f \circ \Phi, g \circ \Phi)\rangle & =\left\langle f_{x} \circ \Phi g \circ \Phi-g_{x} \circ \Phi f \circ \Phi, f_{y} \circ \Phi g \circ \Phi-g_{y} \circ \Phi f \circ \Phi\right\rangle \\
& =\left\langle\left(f_{x} g-g_{x} f\right) \circ \Phi,\left(f_{y} g-g_{y} f\right) \circ \Phi\right\rangle,
\end{aligned}
$$




\section{Milnor Number and Meromorphic Germs}

which implies the first assertion. If $u \in \mathbb{C}\{x, y\}$ one checks

$$
\langle\omega(u f, u g)\rangle=\left\langle u^{2}\left(f_{x} g-f g_{x}\right), u^{2}\left(f_{x} g-f g_{y}\right)\right\rangle,
$$

which equals $\langle\omega(f, g)\rangle$ in case $u$ is a unit. The assertion for $v(f, g)$ follows easily as well.

Another simple but important observation is:

Proposition 3.12. Let $f, g \in \mathbb{C}\{x, y\}$ and $A=\left(\begin{array}{ll}a & b \\ c & d\end{array}\right) \in \mathrm{GL}_{2} \mathbb{C}$. Then

$$
\mu(a f+b g, c f+d g)=\mu(f, g)
$$

and the same property holds for $v$.

Proof. One checks that under the transformation $(f, g) \mapsto A(f, g)$, the distinguished generators of the ideal $\langle\omega(f, g)\rangle$ are mapped to their $(a d-b c)$ multiples.

If one of the functions $f$ or $g$ is nonzero at the origin, then the meromorphic Milnor number reduces to the holomorphic case. Therefore the interesting case is when both germs $f$ and $g$ vanish at the origin.

Proposition 3.13. Let $f, g \in \mathbb{C}\{x, y\}$. If $f(\mathbf{0}) \neq 0$ but $g(\mathbf{0})=0$, then $\mu(f, g)=$ $v(f, g)=\mu(g)$ (maybe infinite).

Proof. It is clear that if $f(\mathbf{0}) \neq 0$ or $g(\mathbf{0}) \neq 0$, we have $\mu(f, g)=v(f, g)$. If we assume $f(\mathbf{0}) \neq 0$ then the ideals $\langle\omega\rangle,\left\langle f^{-2} w\right\rangle$ are the same and the latter can be written as $\langle\partial(g / f)\rangle$. Hence $\mu(f, g)=\mu(g / f)$. Furthermore we have

$$
\mu(g / f)=\mu(g / f-g(\mathbf{0}) / f(\mathbf{0}))=\mu\left(\frac{g f(\mathbf{0})-g(\mathbf{0}) f}{f(\mathbf{0}) f}\right) .
$$

Now this quotient is zero at the origin, so that by the invariance of the Milnor number under the action of the contact group we obtain $\mu(f, g)=\mu(g f(\mathbf{0})-$ $g(\mathbf{0}) f)$. In the case $g(\mathbf{0})=0$, this reduces to the asserted equality.

The proof shows that if $f, g$ both do not vanish at the origin, then we can only conclude $\mu(f, g)=v(f, g)=\mu(g f(\mathbf{0})-g(\mathbf{0}) f)$. 
In the sequel we like to obtain an equivalent characterization of those pairs $(f, g)$ for which $\mu(f, g)$ or $v(f, g)$ is finite. By the results from above we may assume throughout that $f(\mathbf{0})=g(\mathbf{0})=0$.

Proposition 3.14. Let $0 \not \equiv f, g \in \mathbb{C}\{x, y\}$ be vanishing at the origin. Then

i) If $f$ or $g$ is not reduced, then $V(\omega)$ is not just the origin.

ii) If $f, g$ have a common divisor $h$, then $V(\omega(f, g))=V(h) \cup V(\omega(f / h, g / h))$.

iii) If $(f, g)=1$ and $f$, $g$ are reduced, then the following implication is valid:

$$
V(\omega) \subset V(f) \cup V(g) \Rightarrow V(\omega) \subset V(f) \cap V(g)=\{\mathbf{0}\} .
$$

iv) If $f, g$ are coprime, then the following is equivalent:

a) $V(\omega) \subset V(f) \cup V(g)$

b) For every $c \in \mathbb{C}^{*}$, the germ $f-$ cg is reduced.

Proof.

i) If $f$ is nonreduced we can write $f=p^{m} q$ with $p, q \in \mathbb{C}\{x, y\}, p(\mathbf{0})=0$ and $m \geq 2$. The computation

$$
\begin{aligned}
f \partial g-g \partial f & =p^{m} q \partial g-g m p^{m-1} q \partial p-g p^{m} \partial q \\
& =p^{m-1}(p q \partial g-g m q \partial p-g p \partial q)
\end{aligned}
$$

shows that $V(p) \subset V(w)$.

ii) Let $f$ and $g$ have a common divisor $h$, then we have a factorization $f=$ $f_{1} h, g=g_{1} h$ and can compute

$$
\begin{aligned}
f \partial g-g \partial f & =f_{1} h\left(h \partial g_{1}+g_{1} \partial h\right)-g_{1} h\left(h \partial f_{1}+f_{1} \partial h\right) \\
& =h^{2}\left(f_{1} \partial g_{1}-g_{1} \partial f_{1}\right) .
\end{aligned}
$$

iii) Let $V(\omega) \subset V(f) \cup V(g)$. Then

$$
\begin{aligned}
V(\omega) & =V(\omega) \cap(V(f) \cup V(g)) \\
& =V(\omega) \cap V(\langle f\rangle \cap\langle g\rangle) \\
& =V(\langle\omega\rangle+\langle f\rangle \cap\langle g\rangle),
\end{aligned}
$$




\section{Milnor Number and Meromorphic Germs}

hence by the Nullstellensatz

$$
\sqrt{\langle\omega\rangle}=\sqrt{\langle\omega\rangle+\langle f\rangle \cap\langle g\rangle} .
$$

If $V(\omega)=\{\mathbf{0}\}$, then there is nothing to show for iii). So we assume $V(\omega) \underset{\ddagger}{\supset}$ $\{0\}$ which means that there is an irreducible nonunit $h$ with $\sqrt{\langle\omega\rangle} \subset\langle h\rangle(h$ can be taken as one of the branches of $V(\omega)$ ). Then by $\left(^{*}\right)$ we have not only $h \mid f_{x} g-f g_{x}$ and $h \mid f_{y} g-f g_{y}$ but also $h \mid f g$. Since $h \mid f g$, the irreducible $h$ must be an irreducible factor of $f$ or $g$ up to a unit, say $h=f_{1}$. Then $f_{1} \mid f_{x} g-$ $f g_{x}, f_{y} g-f g_{y}$ implies $f_{1} \mid f_{x} g, f_{y} g$ and from $\operatorname{gcd}(f, g)=1$ we get $f_{1} \mid f_{x}, f_{y}$. But then $f$ would not have an isolated singularity at the origin, hence would not be reduced. A contradiction!

$i v$ ) We show the implication $a$ ) $\Rightarrow b$ ) by contraposition. Assume that there is $c \in \mathbb{C}^{*}$ with $f-c g=: h$ nonreduced. The germ $h$ can't be identically zero since $\operatorname{gcd}(f, g)=1$. We compute

$$
\begin{aligned}
f \partial g-g \partial f & =(c g+h) \partial g-g \partial(c g+h) \\
& =h \partial g-g \partial h .
\end{aligned}
$$

Since $h$ is nonreduced it follows already from the item $i)$ that $V(\omega) \supset V\left(h_{1}\right)$ where $h_{1}$ is an irreducible nonunit and $h_{1}^{2}$ divides $h$. In particular $V(w) \subset$ $V(f) \cup V(g)$ can't be true since otherwise for example $V\left(h_{1}\right) \subset V(f)$, so $h_{1}$ divides $f$, but since $h=f-c g, f$ and $g$ would not be coprime.

Finally, we show $b) \Rightarrow a$ ), again by contraposition. So we assume that it is not true that $V(\omega) \subset V(f) \cup V(g)$. If $V(\omega)$ is two-dimensional, then $f_{x} g-f g_{x}$ and $f_{y} g-f g_{y}$ are identically zero in a neighbourhood of the origin. Then $f / g$ would be a locally constant, hence constant function on the connected $\left(\mathbb{C}^{2}, 0\right) \backslash V(g)$, say equal to $c \in \mathbb{C}$. Hence $f-c g=0$ on $\left(\mathbb{C}^{2}, 0\right) \backslash V(g)$ and therefore on all of $\left(\mathbb{C}^{2}, 0\right)$ by continuity, which contradicts $(f, g)=1$ or (if $c=0$ ) $f \not \equiv 0$.

In the remaining case, $V(\omega)$ is one-dimensional and by the contraposition hypothesis it is not true that $V(\omega) \subset V(f) \cup V(g)$. Then there must be a onedimensional irreducible component $V$ of $V(w)$ with the property $V \cap V(f)=$ $V \cap V(g)=\{\mathbf{0}\}$. First of all note, since $V$ is irreducible, $V^{*}:=V \backslash\{\mathbf{0}\}$ is smooth and connected. Since $V \subset V(\omega)$ we have $d(f / g)=0$ on $V^{*}$. Therefore $f / g$ is 
a locally constant function, hence constant on $V^{*}$, so equal to some $c \in \mathbb{C}^{*}$. Let $h=f-c g$. On $V^{*}$ we have

$$
\partial h=\partial f-c \partial g=\partial f-\frac{f}{g} \partial g=\frac{g \partial f-f \partial g}{g^{2}} .
$$

Hence $V(\partial h) \cap V^{*}=V(\omega) \cap V^{*}=V^{*}$ which implies $V(\partial h)$ cannot be $\varnothing$ or $\{\mathbf{0}\}$, hence $h$ could not be an isolated singularity, i.e. is not reduced.

As a corollary to the proof we get the well-known result

Proposition 3.15. Given coprime $0 \not \equiv f, g \in \mathbb{C}\{x, y\}$ with $f(\mathbf{0})=g(\mathbf{0})=0$, the set

$\mathscr{B}_{n i}(f, g):=\left\{(s: t) \in \mathbb{P}^{1} \mid s f+t g\right.$ does not have an isolated singularity at the origin $\}$ is finite.

The subscript $n i$ stands for nonisolated.

Proof. Let the $c_{i}$ 's be pairwise different complex numbers such that $f-c_{i} g$ is not reduced. From the proof above, part (iv), (a) $\Rightarrow$ (b), we infer that $V(\omega) \supset$ some branch of $\left(f-c_{i} g\right)$ for all $i$. Since the case $V(\omega)=\left(\mathbb{C}^{2}, 0\right)$ does not occur (see above proof), $V(\omega)$ is a curve or lower-dimensional. As a curve it can have only finitely many branches. However the $f-c_{i} g$ 's are pairwise coprime. Hence the number of such $c_{i}$ 's is finite.

It is known that for an ideal $I \subset \mathbb{C}\{x, y\}$ we have $\operatorname{dim}(\mathbb{C}\{x, y\} / I)<\infty$ if and only if $V(I) \subset\{0\}$ (this follows e.g. by using cor. 1.74 of [GLS07 for the coherent sheaf $\mathscr{O} / \mathscr{I})$. If we apply this to the ideal $\langle\omega(f, g)\rangle$ we find that proposition 3.14 gives a criterion for the finitenss of $\mu(f, g)$ which we will summarize in the next theorem.

Before that we are concerned with the finiteness of $v(f, g)$. That's why we assume $v(f, g)<\infty$ but $\mu(f, g)=\infty$. (It is clear that $\mu(f, g)<\infty$ would imply $v(f, g)<\infty)$. In this case we must have $i(f, g)=\infty$, otherwise $\mu(f, g)=$ $i(f, g)+v(f, g)$ would be finite.

The intersection number of $f$ and $g$ is infinite if and only if their greatest common divisor $h$ is a nonunit. Writing $f=\tilde{f} h, g=\tilde{g} h$, we have $\omega(f, g)=$ 


\section{Milnor Number and Meromorphic Germs}

$h^{2} \omega(\tilde{f}, \tilde{g})=h^{2} \tilde{\omega}$. Hence we obtain an isomorphism of vector spaces (by dividing by $h$ ):

$$
\frac{\langle f, g\rangle}{\langle\omega\rangle} \cong \frac{\langle\tilde{f}, \tilde{g}\rangle}{\langle h \tilde{\omega}\rangle} .
$$

Make clear that we have an exact sequence of the form

$$
0 \rightarrow \frac{\langle\tilde{\omega}\rangle}{\langle h \tilde{\omega}\rangle} \rightarrow \frac{\langle\tilde{f}, \tilde{g}\rangle}{\langle h \tilde{\omega}\rangle} \rightarrow \frac{\langle\tilde{f}, \tilde{g}\rangle}{\langle\tilde{\omega}\rangle} \rightarrow 0 .
$$

So since the middle-term vector space is finite-dimensional, so are the other two. Since now $\tilde{f}$ and $\tilde{g}$ are coprime we have $i(\tilde{f}, \tilde{g})<\infty$. And because the right vector space is finite-dimensional, we deduce $\mu(\tilde{f}, \tilde{g})<\infty$. The latter is just $\operatorname{dim}_{\mathbb{C}} \mathbb{C}\{x, y\} /\langle\tilde{\omega}\rangle$. But since the vector space to the left is also finite-dimensional, so would be $\operatorname{dim}_{\mathbb{C}} \mathbb{C}\{x, y\} /\langle h \tilde{\omega}\rangle$. This can't be true since $h$ is a nonunit! Hence we get a contradiction showing that $\mu(f, g)=\infty$ and $v(f, g)<\infty$ cannot hold simultaneously. We have proved:

Theorem 3.16. Let $0 \not \equiv f, g \in \mathbb{C}\{x, y\}$ and assume $f(\mathbf{0})=g(\mathbf{0})=0$. Then the following statements are equivalent:

1. The number $\mu(f, g)$ is finite.

2. The number $v(f, g)$ is finite.

3. The germs $f$, g are coprime and every linear combination $s f+t g$ (with $\left.(s: t) \in \mathbb{P}^{1}\right)$ is a reduced germ.

The simplest example for an infinite $\mu(f, g)$ is given by $f(x, y)=x, g(x, y)=$ $x-y^{2}$ in which case $\langle\omega\rangle=\left\langle y^{2}, x y\right\rangle$ and $V(\omega)=V(y)$ is not just the origin. Another example is $f(x, y)=x^{2}-y^{2}$ and $g(x, y)=x^{2}+y^{2}$, where $\langle\omega\rangle=\left\langle x^{2} y, x y^{2}\right\rangle$ so that $V(\omega)=V(x y)$.

Proposition 3.17. Let $p, q \in \mathfrak{m}_{\mathbb{C}^{2}, 0}$ be two homogeneous polynomials of the same degree. Then $\mu(p, q)=1$ or $\mu(p, q)=\infty$.

Proof. Let $d$ be the degree of $p$ and $q$. If we had $\mu(p, q)<\infty$, then all $s p+$ $t q$ are isolated singularities and homogeneous of degree $d$. But it is wellknown that in this case the Milnor number is given by $(d-1)^{2}$ for all $(s, t)$. 


\subsection{Milnor Number of Pairs of Functions}

Hence there is no jump in the Milnor number along the pencil at all, meaning $\mathscr{B}(p, q)=\varnothing$, hence $(p, q) \sim(x, y)$, thus $\mu(p, q)=1$.

We have given a definition of a "Milnor number" of a pair of function germs defined on two-dimensional complex space. How could we define a similar number for higher dimensions? Let $f_{1}, \ldots, f_{n}:\left(\mathbb{C}^{n}, 0\right) \rightarrow(\mathbb{C}, 0)$ be holomorphic germs defined in a neighbourhood of the origin in $\mathbb{C}^{n}$. I suggest to use the following definition

$$
v\left(f_{1}, \ldots, f_{n}\right)=\operatorname{dim}_{\mathbb{C}} \frac{\left\langle f_{1} \cdots \widehat{f}_{i} \cdots f_{n}\right\rangle_{i=1}^{n}}{\left\langle\sum_{i=1}^{n}(-1)^{i} \partial f_{i} \cdot f_{1} \cdots \widehat{f}_{i} \cdots f_{n}\right\rangle_{\partial=\partial_{1}}^{\partial_{n}}} .
$$

If the common zero locus of the $f_{i}$ 's is merely the origin, I suggest to define

$$
\mu\left(f_{1}, \ldots, f_{n}\right)=v\left(f_{1}, \ldots, f_{n}\right)+i\left(f_{1}, \ldots, f_{n}\right),
$$

where $i\left(f_{1}, \ldots, f_{n}\right)=\operatorname{dim}_{\mathbb{C}} \mathbb{C}\left\{x_{1}, \ldots, x_{n}\right\} /\left\langle f_{1}, \ldots, f_{n}\right\rangle$. Then for $f_{1}=x_{1}, \ldots, f_{n}=$ $x_{n}$ we obtain $\mu\left(f_{1}, \ldots, f_{n}\right)=1$ as was the case when $n=2$.

In the sequel we are going to classify pairs $(f, g)$ with a given $\mu(f, g) \leq 2$.

Since $\langle\omega\rangle=\left\langle f_{x} g-f g_{x}, f_{y} g-f g_{y}\right\rangle$ is a subideal in $\langle f, g\rangle$ we have the following relation:

$$
\begin{aligned}
\operatorname{dim}_{\mathbb{C}} \frac{\mathbb{C}\{x, y\}}{\langle\omega\rangle} & =\operatorname{dim}_{\mathbb{C}} \frac{\mathbb{C}\{x, y\}}{\langle f, g\rangle}+\operatorname{dim}_{\mathbb{C}} \frac{\langle f, g\rangle}{\langle\omega\rangle}, \\
\mu(f, g) & =i(f, g)+v(f, g) .
\end{aligned}
$$

This immediately produces the following table with the help of which the classification will be easy.

\begin{tabular}{ccc}
$\mu(f, g)$ & $i(f, g)$ & $v(f, g)$ \\
\hline \hline 0 & 0 & 0 \\
\hline 1 & 1 & 0 \\
1 & 0 & 1 \\
\hline 2 & 2 & 0 \\
2 & 1 & 1 \\
2 & 0 & 2 \\
\hline
\end{tabular}




\section{Milnor Number and Meromorphic Germs}

First of all we need the

Lemma 3.18. For $f, g \in \mathbb{C}\{x, y\}$ we have

- $i(f, g)=0$ holds if and only if $f(0) \neq 0$ or $g(0) \neq 0$.

- $i(f, g)=1$ holds if and only if $(f, g)$ is right equivalent to $(x, y)$.

Proof. Although this is known, we give a proof for completeness of the discussion. For the first item we merely note that $i(f, g)=0$ iffif the ideal < $f, g>$ is the full ring $\mathbb{C}\{x, y\}$ which can only happen if one or both of $f, g$ do not vanish at the origin. Now for the second item. The relation $i(f, g)=1$ holds iffif $\langle f, g\rangle$ is the maximal ideal. Hence there are $a, b, c, d \in \mathbb{C}\{x, y\}$ with $x=a f+b g$ and $y=c f+d g$. Derive after $x, y$ and insert the origin (use that $f(0)=0, g(0)=0$ by the first item) to get

$$
\left(\begin{array}{ll}
1 & 0 \\
0 & 1
\end{array}\right)=\left(\begin{array}{ll}
f_{x}(0) & g_{x}(0) \\
f_{y}(0) & g_{y}(0)
\end{array}\right)\left(\begin{array}{ll}
a(0) & c(0) \\
b(0) & d(0)
\end{array}\right) .
$$

It follows that $\operatorname{det}(\partial(f, g)(0)) \neq 0$ and the inverse function theorem does the rest.

We assume overall that at least one of $f(0), g(0)$ vanish.

Let $i(f, g)=0$. Then lemma 3.18 and proposition 3.13 show that $\mu(f, g)=$ $\mu(f)$ if $g(0) \neq 0$ (but $f(0)=0$ ).

Case $\mu(f, g)=0$. Then $f$ is smooth and vanishing at the origin. Hence we can transform $(f, g)$ into $(x, h)$ where $h$ is a nonvanishing function at the origin.

Case $\mu(f, g)=1$. Then $f$ is critical, Morse and vanishing at the origin. Hence we can transform $(f, g)$ into $(x y, h)$ where $h$ is a nonvanishing function at the origin.

Case $\mu(f, g)=2$. Then, a well-know result from singularity theory gives that $(f, g)$ is right equivalent to $\left(x^{2}+y^{3}, h\right)$ with $h(0) \neq 0$. 
Now let $i(f, g)=1$. Then $(f, g)$ is right equivalent to $(x, y)$ by lemma 3.18 In this case $\mu(f, g)=1$.

It remains the case where $\mu(f, g)=2=i(f, g)$ and $v(f, g)=0$.

If $v(f, g)=0$, there exists power series $a, b, c, d$ with

$$
\begin{aligned}
& f=a\left(f_{x} g-f g_{x}\right)+b\left(f_{y} g-f g_{y}\right), \\
& g=c\left(f_{x} g-f g_{x}\right)+d\left(f_{y} g-f g_{y}\right),
\end{aligned}
$$

from which we infer

$$
\begin{aligned}
f\left(1+a g_{x}+b g_{y}\right) & =g\left(a f_{x}+b f_{y}\right), \\
g\left(1-c f_{x}-d f_{y}\right) & =-f\left(c g_{x}+d g_{y}\right) .
\end{aligned}
$$

Assume that $f(0)=0, g(0) \neq 0$. Then from the second equation we infer $1-c(0) f_{x}(0)-d f_{x}(0)=0$. Hence, $f$ must be noncritical. Since $g(0) \neq 0$ this is the first line of the table below. Hence there are no occurences. Assume now that both $f(0), g(0)$ vanish. Then $(f, g)=1$ since $\mu(f, g)<\infty$. If $f$ is critical at the origin, then $1-c f_{x}-d f_{y}$ is a unit in the power series ring and from $(f, g)=1$ we get a contradiction. The same with $g$. Hence $f, g$ are both noncritical and vanish at the origin. Hence we can transform $(f, g)$ to either $(x, y)$ or $(x, x+h)$ where $h \in \mathfrak{m}^{2}$. The first case cannot occur since then $i(f, g)=1$. In the second case it follows from the later proposition 3.27 that $\mu(f, g)=\mu(x, h)=\mu(h)+2 * i(x, h)-1$ which yields the contradiction $2=$ $\mu(h)+2 * 2$. We summarize this short computations in the next proposition.

Proposition 3.19. When $f(0) g(0)=0$ we have the following classification table (up to right equivalence of pairs) where $h \in \mathbb{C}\{x, y\}$ with $h(0) \neq 0$ :

\begin{tabular}{cccc}
$\mu(f, g)$ & $i(f, g)$ & $v(f, g)$ & $(f, g)$ \\
\hline \hline 0 & 0 & 0 & $(x, h)$ or $(h, x)$ \\
\hline 1 & 1 & 0 & $(x, y)$ \\
1 & 0 & 1 & $(x y, h)$ or $(h, x y)$ \\
\hline 2 & 2 & 0 & void \\
2 & 1 & 1 & void \\
2 & 0 & 2 & $\left(x^{2}+y^{3}, h\right)$ or $\left(x^{2}+y^{3}, h\right)$ \\
\hline
\end{tabular}




\section{Milnor Number and Meromorphic Germs}

We note that $h$ cannot be further specified. There are too many equivalence classes. For example the pairs $\left(x, y^{m}+1\right)$ and $\left(x, y^{n}+1\right)$ with different $m, n$ are not right equivalent. Namely if if $\Phi(x, y)=(\phi(x, y), \psi(x, y))$ was such a transformation, then $\phi(x, y)=x$ and from the other equivalence relation $\psi(x, y)^{m}+1=\psi(x, y)^{n}+1$ we would get $\psi(x, y)^{m-n}=1$, so that $\psi(x, y)$ would be constant, which is impossible.

The following proposition should be seen as the analogous result to $\mu(f)=$ 1 iffif $f$ Morse-critical. It follows from the above considerations when we note the fact that for $f, g \in \mathbb{C}\{x, y\}$ and $g(0) \neq 0$, we have that $f / g$ is critical at the origin if and only if $f$ is critical. In this situation if furthermore $f(0)=0$, then $f / g$ is Morse if and only if $f$ is Morse (at the origin).

Proposition 3.20. Let $f, g \in \mathbb{C}\{x, y\}$ with $f(0) g(0)=0$. Then $\mu(f, g)=1$ if and only if we are in one of the following cases:

- The pair $(f, g)$ is right equivalent to $(x, y)$.

- We have $f(0) \neq 0$ and $g / f$ is a vanishing Morse-critical germ at the origin.

- We have $g(0) \neq 0$ and $f / g$ is a vanishing Morse-critical germ at the origin.

Proposition 3.21 (Deformation property of $\mu(-,-)$ ).

Let $f=f(x, y), g=g(x, y) \in \mathfrak{m}_{\mathbb{C}^{2}, 0}$ with $\mu(f, g)<\infty$. Let $f(x, y, t), g(x, y, t)$ be unfoldings of $f(x, y), g(x, y)$. Then for any sufficiently small neighbourhood $U$ of the origin in $\mathbb{C}^{2}$ we have for each sufficiently small $t \in \mathbb{C}, t \approx 0$

$$
\mu(f, g)=\sum_{p \in U_{t}} \mu_{p}(f(x, y, t), g(x, y, t))
$$

where $U_{t}:=\left\{(x, y) \in U \mid f_{x}(x, y, t) g(x, y, t)-f(x, y, t) g_{x}(x, y, t)=0, f_{y}(x, y, t) g(x, y, t)-\right.$ $\left.f(x, y, t) g_{y}(x, y, t)=0\right\}$.

Proof. The proof of such statements is nowadays standard. First, a sufficiently small representative of the map

$$
\begin{aligned}
\left(\mathbb{C}^{3}, 0\right) \rightarrow\left(\mathbb{C}^{3}, 0\right),(x, y, t) \rightarrow( & f(x, y, t)_{x} g(x, y, t)-f(x, y, t) g_{x}(x, y, t), \\
& \left.f(x, y, t)_{y} g(x, y, t)-f(x, y, t) g_{y}(x, y, t), t\right)
\end{aligned}
$$


is flat and finite. (The zero fibre is just a point, so finiteness follows from the Weierstraß finiteness theorem. Due to the same reason, the map is flat, compare [GLS07], prop 2.7.) From [GLS07], theorem 1.81 (principle of conservation of numbers) applied to the sheaf $\mathscr{O}_{\mathbb{C}^{3}}$ restricted to a small neighbourhood of $\mathbf{0}$ the assertion follows.

\subsection{Singularities in the Pencil}

Let $f, g$ be coprime holomorphic germs at the origin in $\mathbb{C}^{2}$ which vanish at the origin. For any parameter value $(s: t) \in \mathbb{P}^{1}$ we obtain the germ of a holomorphic function $s f+t g:(\mathbb{C}, 0) \rightarrow(\mathbb{C}, 0)$. We know that usually not all pencil members $s f+t g$ have an isolated singularity at the origin. We know a neccessary and sufficient condition for this taking place. With this statement in mind it is worth to look at two other questions which somewhat look similar but are not.

(I) Is there a neighbourhood $U$ of the origin in $\mathbb{C}^{2}$ such that all functions $s f+t g: U \rightarrow \mathbb{C}$ have no critical point except the origin?

(II) Is there a neighbourhood $U$ of the origin in $\mathbb{C}^{2}$ such that all varieties $\{\mathbf{x} \in U \mid s f(\mathbf{x})+\operatorname{tg}(\mathbf{x})=0\}$ are smooth except at the origin?

We come to question (I). The answer is no, as can be seen by the following example. Let $f(x, y):=x y, g(x, y):=x^{2}+y^{2}+x^{3}+y^{3}$. If $(x, y)$ is a critical point of $s f+t g$, then

$$
\begin{aligned}
& s y+t\left(2 x+3 x^{2}\right)=0 \\
& s x+t\left(2 y+3 y^{2}\right)=0
\end{aligned}
$$

When we multiply the first equation with $x$ and the second with $y$ and take differences, we get $t\left(2 x^{2}+3 x^{3}-2 y^{2}-3 y^{3}\right)=0$. If $t \neq 0$, this is equivalent to

$$
\left[2(x+y)+3\left(x^{2}+x y+y^{2}\right)\right](x-y)=0 .
$$

If we take the solution $x=y$ of this equation and insert it into one of the two equations above, we get $s x+t\left(2 x+3 x^{2}\right)=0$, hence either $x=0$ (which would 


\section{Milnor Number and Meromorphic Germs}

imply $y=0$, so the point is the origin) or

$$
(x(t), y(t))=\left(-\frac{s+2 t}{3 t},-\frac{s+2 t}{3 t}\right)
$$

would be a critical point of $s f+t g$. As $(s: t)$ approaches $(-2: 1)$, the point $(x(t), y(t))$ tends to the origin. This already shows the assertion. On the other hand, it is interesting to note that $(-2: 1)$ is one of the bifurcation values. I believe that this is a general phenomenon:

Conjecture 3.22. If a sequence of points $\mathbf{x}_{k} \neq 0$ converges to the origin $0 \in \mathbb{C}^{2}$ and if there are $\left(s_{k}: t_{k}\right) \in \mathbb{P}^{1}$ such that $\mathbf{x}_{k}$ is a critical point of $s_{k} f+t_{k} g$, then $\left(s_{k}: t_{k}\right)$ has as accumulation points only values in $\mathscr{B}(f, g)$.

I thought of a proof using the Łojasiewicz-inequality. For example it is known ([Lê73] $)$ that for a $\mu$-constant family of isolated singularities

$$
f+\operatorname{tg}:\left(\mathbb{C}^{2} \times \mathbb{C}, 0\right) \rightarrow(\mathbb{C}, 0)
$$

there exist a neighbourhood $U$ of $0 \in \mathbb{C}^{2}$ such that for all small $t$ the following holds. When $\partial_{x}(f+t g)=0$ and $\partial_{y}(f+t g)=0$, then $g=0$. But this does not bring us further. Maybe it follows from a characterization of the bifurcation locus using the gradient.

Let us approach question (II).

Proposition 3.23. If $\mu(f, g)<\infty$ there is a neighbourhood $U$ of the origin $0 \in \mathbb{C}^{2}$ such that every variety $\{\mathbf{x} \mid s f(\mathbf{x})+\operatorname{tg}(\mathbf{x})=0\}$ is smooth in $U \backslash\{0\}$.

I cannot find an example where the condition $\mu(f, g)<\infty$ is really necessary.

Proof. Let $\mathbf{x}$ be a nonzero point in $\left(\mathbb{C}^{2}, 0\right)$ and $(s: t) \in \mathbb{P}^{1}$ such that

$$
\begin{array}{r}
s f(\mathbf{x})-\operatorname{tg}(\mathbf{x})=0, \\
\partial_{x}(s f-\operatorname{tg})(\mathbf{x})=0, \\
\partial_{y}(s f-\operatorname{tg})(\mathbf{x})=0 .
\end{array}
$$


Assume without loss of generality $s \neq 0$ and put $\lambda=t / s$. We compute

$$
\begin{aligned}
& \left(f_{x} g-f g_{x}\right)(\mathbf{x})=\left(\lambda g_{x} g-f g_{x}\right)(\mathbf{x})=g_{x}(\mathbf{x})(\lambda g-f)(\mathbf{x})=0 \\
& \left(f_{y} g-f g_{y}\right)(\mathbf{x})=\left(\lambda g_{y} g-f g_{y}\right)(\mathbf{x})=g_{y}(\mathbf{x})(\lambda g-f)(\mathbf{x})=0 .
\end{aligned}
$$

On the other hand since $\mu(f, g)<\infty$ there is a neighbourhood of the origin such that $f_{x} g-f g_{x}, f_{y} g-f g_{y}$ have no common nonzero solution. This completes the proof.

In the next statement we show that the bifurcation set can have any desired cardinality even with the assumption that $\mu(f, g)<\infty$.

Proposition 3.24. Let $n \geq 0$ be a given integer.

(a) Then there exists $f, g \in \mathfrak{m}$ such that the pencil generated by $f$ and $g$ has precisely $n$ nonisolated members and all other members have the same Milnor number.

(b) Then there exists $p, q \in \mathfrak{m}$ with $\mu(p, q)<\infty$ such that $\mathscr{B}(p, q)$ has cardinality $n$.

Proof. If $n=0$ we can take $(f, g)=(p, q)=(x, y)$. If $n=1$, then $(f, g)=$ $\left(x, x+y^{2}\right)$ resp. $(p, q)=\left(x, x+x^{2}+y^{2}\right)$ will provide examples for $(a),(b)$ respectively. Assume $n \geq 2$ from now on. For part $(a)$ we let $f(x, y)=(n-1) x^{n}$ and $g(x, y)=y^{n}-n y x^{n-1}$. If $(s: t)=(1: 0)$ we have $s f+t g=(n-1) x^{n}$ which is a nonisolated singularity, for $n \geq 2$. Let now $t \neq 0$. Then we may put $\lambda:=s / t$ and compute the partial derivatives

$$
\begin{aligned}
& (\lambda f+g)_{x}=n(n-1)\left(\lambda x^{n-1}-y x^{n-2}\right) \\
& (\lambda f+g)_{y}=n\left(y^{n-1}-x^{n-1}\right) .
\end{aligned}
$$

So if both derivatives vanished at $(x, y)$, then from the second equation we would get $\xi x=y$ for some $\xi \in \mathbb{C}$ with $\xi^{n-1}=1$. Inserting this into the first equation gives

$$
\begin{aligned}
\lambda x^{n-1}-y x^{n-2} & =0 \\
\lambda x^{n-1}-\xi x^{n-1} & =0 \\
\Rightarrow x^{n-1}(\lambda-\xi) & =0 .
\end{aligned}
$$




\section{Milnor Number and Meromorphic Germs}

So if $\lambda$ is not an $(n-1)$ st root of unity then any common solution $(x, y)$ of $(\lambda f+g)_{x}=0$ and $(\lambda f+g)_{y}=0$ is only the origin itself. I.e. we have an isolated singularity. Hence the values of $(s: t)$ for which $s f+t g$ is a nonisolated singularity are thus given by $(1: 0)$ and $(\xi: 1)$ where $\xi$ goes through the $(n-1)$ st roots of unity. At all other values of $(s: t)$ we have an isolated singularity given by a homogeneous polynomial of degree $n$. By a result of Milnor and Orlik any such singularity has Milnor number $(n-1)^{2}$. In particular the Milnor numbers are equal for those $(s: t)$. This proves part $(a)$ of the assertion.

Now we prove part $(b)$. When $n=2$ we can use $(p, q)=\left(x^{2}+y^{3}, x^{3}+y^{2}\right)$ where obviously the special pencil members are $p$ and $q$ with Milnor numbers both equal to 2 and all other pencil members have Milnor number equal to 1 . Finally we have $\mu(p, q)=11<\infty$.

From now on assume $n \geq 3$. For any $m \geq(n-1)^{2}+1$ with the previously defined $f, g$ the function pair

$$
(p, q)=\left(f+(n-1) y^{m}, g\right)=\left((n-1)\left(x^{n}+y^{m}\right), y^{n}-n y x^{n-1}\right)
$$

is as desired.

First note that $m>n$ since $n \geq 3$. We show that the germs $x^{n}+y^{m}$ and $y^{n}-n y x^{n-1}$ are coprime. In general $x^{n}+y^{m}=\prod_{\xi}\left(x^{n / d}-\xi y^{m / d}\right)$ where the product is over the $d$ th roots of -1 and $d=\operatorname{gcd}(m, n)$. So for a branch of $x^{n}+y^{m}$ we have a parametrization of the form $\gamma(t)=\left(\sqrt[n / d]{\xi} t^{m / d}, t^{n / d}\right)$ which does not kill $\left(y^{n}-n y x^{n-1}\right) \circ \gamma(t)$ as is easily checked.

The germ $p$ is an isolated singularity with Milnor number $(n-1)(m-1)$. Now define $\lambda=s / t$ and compute the derivatives

$$
\begin{aligned}
(\lambda p+q)_{x} & =\lambda(n-1) n x^{n-1}-n(n-1) y x^{n-2} \\
& =n(n-1) x^{n-2}(\lambda x-y), \\
(\lambda p+q)_{y} & =\lambda(n-1) m y^{m-1}+n y^{n-1}-n x^{n-1} .
\end{aligned}
$$

Let $(x, y)$ be a critical point of $\lambda p+q$. Then we have either $x=0$ or $\lambda x-y=0$. 
In the first case we obtain $\lambda(n-1) m y^{m-1}+n y^{n-1}=0$ which can be rewritten as $y^{n-1}\left(\lambda(n-1) y^{m-n}+n\right)=0$. Since $m>n$, the expression in brackets does not vanish at the origin and so $y=0$, in which case we would get an isolated singularity of $\lambda p+q$. In the second case we have $\lambda x-y=0$. Inserting this into the equation for $\partial_{y}(\lambda p+q)$ we get

$$
\begin{aligned}
\lambda(n-1) m(\lambda x)^{m-1}+n(\lambda x)^{n-1}-n x^{n-1} & =0 \\
x^{n-1}\left((n-1) m \lambda^{m} x^{m-n}+n \lambda^{n-1}-n\right) & =0 .
\end{aligned}
$$

We again have two cases. Either $\lambda^{n-1}=1$ or not. If $\lambda^{n-1} \neq 1$, then the expression in brackets is nonvanishing at the origin. So $x=0$ and therefore $y=\lambda x=0$, too. In the case $\lambda^{n-1}=1$, we rewrite the last equation as

$$
x^{m-1}\left((n-1) m \lambda^{m}\right)=0 .
$$

which implies $x=0$, too. So for any $\lambda \in \mathbb{C}, \lambda p+q$ has an isolated singularity at the origin. Since this holds also for $\lambda=\infty$ (i.e. for $p$ ), we have $\mu(p, q)<$ $\infty$ by theorem 3.16 . What about the bifurcation set? We know by part ( $a$ ) that the Milnor number of $\lambda f+g$ is equal to $(n-1)^{2}$ for $\lambda \in \mathbb{C}$ not an $(n-$ 1)th root of unity. Since $m \geq \mu(\lambda f+g)+1$, by Tougeron's finite determinacy result adding $\lambda(n-1) y^{m}$ to the isolated singularity $\lambda f+g$ will not change the Milnor number. So the generic Milnor number of the pencil generated by $p, q$ is $\mu_{g e n}(p, q)=(n-1)^{2}$ and it remains to compare the Milnor number of $p$ resp. $\lambda p+q$ for $\lambda^{n-1}=1$ with $(n-1)^{2}$. For $p$ we have $\mu(p)=(n-1)(m-1)>$ $(n-1)^{2}$, so $(s: t)=(1: 0)$ belongs to $\mathscr{B}(p, q)$. Finally let $\lambda^{n-1}=1$. We have

$$
\begin{aligned}
\mu(\lambda p+q)= & i\left(\lambda p_{x}+q_{x}, \lambda p_{x}+q_{x}\right) \\
= & i\left(x^{n-2}(\lambda x-y), \lambda(n-1) m y^{m-1}+n y^{n-1}-n x^{n-1}\right) \\
= & (n-2) i\left(x, \lambda(n-1) m y^{m-1}+n y^{n-1}-n x^{n-1}\right) \\
& +i\left(\lambda x-y, \lambda(n-1) m y^{m-1}+n y^{n-1}-n x^{n-1}\right) \\
= & (n-2) \operatorname{ord}_{t}\left(-n t^{n-1}\right)+\operatorname{ord}_{t}\left(\lambda(n-1) m(\lambda t)^{m-1}+n(\lambda t)^{n-1}-n t^{n-1}\right) \\
= & (n-2)(n-1)+(m-1) \\
= & (n-1)^{2}+(m-n) \\
> & (n-1)^{2}=\mu_{g e n}(f, g) .
\end{aligned}
$$


So $(\lambda: 1) \in \mathscr{B}(p, q)$. Altogether we deduce that $\mathscr{B}(p, q)$ has $n$ elements.

\subsection{The Bifurcation Formula}

Conjecture 3.25 (Bifurcation Formula). Assume that $f, g \in \mathbb{C}\{x, y\}$ both vanish at the origin and that $\mu(f, g)$ is finite. If $\mu_{g e n}$ denotes the generic Milnor number and $\mathscr{B}^{*}=\mathscr{B}(f, g) \backslash\{0, \infty\}$ the reduced bifurcation set of the local pencil generated by $f$ and $g$ (in this order), then the following relation holds:

$$
\mu(f, g)=\mu(f \cdot g)+\sum_{(s: t) \in \mathscr{B}^{*}}\left(\mu(s f+t g)-\mu_{g e n}\right) .
$$

As a corollary of this conjecture we have

Conjecture 3.26. Under the above hypothesis $\mu(f, g) \geq \mu(f g)$ with equality if and only if the meromorphic germ $f / g$ is semitame (i.e. $\mathscr{B}(f, g) \subset\{0, \infty\})$.

At this point it seems worth to recall the formula

$$
\mu(f g)=\mu(f)+\mu(g)+2 \cdot i(f, g)-1 .
$$

Note that the action of $\operatorname{PGL}(2, \mathbb{C})$ on $\mathbb{P}^{1}=\mathbb{P}\left(\mathbb{C}^{2}\right)$ makes it possible to normalize the bifurcation set: three different members of $B$ can be assumed to be $\{0,1, \infty\}$ by changing $(f, g)$ to some $(a f+b g, c f+d g)$. If we write $\mathscr{B}=\left\{\left(s_{1}: t_{1}\right), \ldots\right\}$ as a subset of $\mathbb{P}^{1}$ then the map $A \in \operatorname{PGL}(2, \mathbb{C})$ can be explicitely given by

$$
A=\frac{1}{s_{1} t_{2}-s_{2} t_{1}}\left(\begin{array}{ll}
\lambda s_{1} & \mu s_{2} \\
\lambda t_{1} & \mu t_{2}
\end{array}\right) \text { where } \lambda=s_{3} t_{2}-s_{2} t_{3} \text { and } \mu=s_{3} t_{1}-s_{1} t_{3} .
$$

We give some examples with shifting the bifurcation set. Let $p(x, y)=$ $y^{4}+x y^{2}+x^{3}$ and $q(x, y)=x y^{2}+x^{2} y+x^{3}$. If $f=p, g=q$, then $\mathscr{B}=\left\{-\frac{2}{3},-2,-1\right\}$ and the above formula reads $30=27+(5-4)+(5-4)+(5-4)$.

If $f=p, g=q-p$, then $\mathscr{B}=\{-2,2, \infty\}$ and the above formula is $30=$ $28+(5-4)+(5-4)$. 
If $f=q-p, g=p$ (so interchanging $f, g$ from the last example) then $\mathscr{B}=$ $\left\{0,-\frac{1}{2}, \frac{1}{2}\right\}$ and the formula is $30=28+(5-4)+(5-4)$.

Finally if $f=3 p-2 q, g=-p+2 q$, then $\mathscr{B}=\{0,-1, \infty\}$ and the formula reads $30=29+(5-4)$.

Note that the preceding three examples clearly show that one needs to exclude 0 and $\infty$ from the bifurcation set in the formula.

Here is an example with higher Milnor numbers:

$$
\begin{aligned}
& f(x, y)=-y^{4}+y^{5}-x y^{4}+x^{5}, g(x, y)=\frac{1}{2} y^{5}+\frac{1}{2} x^{3}+\frac{1}{2} x^{5}, \\
& \mathscr{B}(f, g)=\{0, \infty\}, \mu(f)=12, \mu(g)=8, \mu_{g e n}=6, \mu(f, g)=\mu(f g)=43 .
\end{aligned}
$$

Proposition 3.27. The bifurcation formula holds when one of $f, g$ is a smooth germ.

Proof. Let $f$ be smooth. After a linear coordinate transformation we can assume $f(x, y)=x$. If $g$ is smooth, too, it is either transversal to $f$ or not. The case of smooth transversals $f, g$ is trivial since $\mathscr{B}(f, g)=\varnothing$ and $\mu(f g)=1$ as well as $\mu(x, y)=1$. In the second case $g(x, y)$ is a complex multiple of $x$ so that $f, g$ are not coprime, which contradicts the hypothesis. Now let $g$ be nonsmooth. Then $g \in \mathfrak{m}^{2}$. Then $\mu(f, g)<\infty$ if and only if $x$ does not divide $g$ and $g$ is reduced. So let us assume this.

Since all germs $s f+t g=s x+t g$ are smooth except the one with $s=0$, we have $\mathscr{B}(x, g)=\{\infty\}$, so that $\mathscr{B}^{*}(x, g)=\mathscr{B}(x, g) \backslash\{0, \infty\}=\varnothing$. By the general formula $\mu(x g)=\mu(x)+\mu(g)+2 i(x, g)-1$, the asserted formula is

$$
\mu(x, g)=\mu(g)+2 i(x, g)-1+0 .
$$

Now it is easily checked that $\mu(x, g)=i\left(g-x g_{x}, x g_{y}\right)$ so that the formula to be shown is equivalent to

$$
i\left(g-x g_{x}, x g_{y}\right)=\mu(g)+2 i(x, g)-1 .
$$

We now apply a special case of Lê's formula 3.4 (applied to the pair $(g, x)$ ) 
$\mu(g)+i(x, g)-1=i\left(g, g_{y}\right)$ to get the equivalent statements

$$
\begin{aligned}
i\left(g-x g_{x}, g_{y}\right)+i\left(g-x g_{y}, x\right) & =i\left(g, g_{y}\right)+i(x, g) . \\
i\left(g-x g_{x}, g_{y}\right) & =i\left(g, g_{y}\right)
\end{aligned}
$$

Finally, for a quasihomogeneous germ $g$, this equality is easily seen, while in general this last equation follows from (the proof of) lemma 6.5.7 in [Wal04.

In the remaining part of this section I like to discuss some ideas for a proof of the bifurcation formula.

As part of the big conjecture we have the following

Conjecture 3.28 (Partial Conjecture). If $f, g \in \mathfrak{m}_{\mathbb{C}^{2}, 0}$ with $\mu(f, g)<\infty$ and $\mathscr{B}(f, g) \subset\{0, \infty\}$ then $\mu(f, g)=\mu(f g)$.

I can think of two ways of proving it. The first is to use repeated blowup. The second is to use a homotopy.

For the homotopy approach I suggest to look at the map

$$
[0,1] \times S_{\epsilon}^{3} \rightarrow S^{3}, \quad(\tau, x, y) \mapsto \frac{\left(s(\tau) f_{x} g+t(\tau) f g_{x}, s(\tau) f_{y} g+t(\tau) f g_{y}\right)}{\left\|\left(s(\tau) f_{x} g+t(\tau) f g_{x}, s(\tau) f_{y} g+t(\tau) f g_{y}\right)\right\|} .
$$

Here the path $[0,1] \ni \tau \mapsto(s(\tau), t(\tau)) \in \mathbb{P}^{1}$ has to be chosen to connect the points $(1: 1)$ (corresponding to $\mu(f g))$ and $(1:-1)$ (corresponding to $\mu(f, g)$ ). Of course the above map does not exist in general. If it exists, it will immediately follow that $\mu(f, g)=\mu(f g)$ since the degree is a homotopy invariant. In order to show that it exists one has to pay attention to the fact that $\epsilon$ must not depend on $\tau$, i.e. we make the

Conjecture 3.29. Let $f, g \in \mathfrak{m}_{\mathbb{C}^{2}, 0}, \mu(f, g)<\infty$ and $\mathscr{B}(f, g) \subset\{0, \infty\}$. Then there exists a neighbourhood $U$ of the origin in $\mathbb{C}^{2}$ and a path $(s, t)=(s(\tau), t(\tau))$ connecting $(1: \pm 1)$ such that the common zero locus of $s(\tau) f_{x} g+t(\tau) f g_{x}=0$ and $s(\tau) f_{y} g+t(\tau) f g_{y}$ in $U$ is only the origin itself!

We can make this claim more precise in 
Conjecture 3.30. Under the above hypothesis the following holds for sufficiently small $\epsilon>0$. There does not exist a series $\left(s_{i}: t_{i}\right) \in \mathbb{P}^{1}$ and points $\left(x_{i}, y_{i}\right) \in B_{\epsilon}(0) \backslash\{0\}$ with the following properties

1 .

$$
\begin{aligned}
& s_{i} f_{x}\left(x_{i}, y_{i}\right) g\left(x_{i}, y_{i}\right)+t_{i} f\left(x_{i}, y_{i}\right) g_{x}\left(x_{i}, y_{i}\right)=0, \\
& s_{i} f_{y}\left(x_{i}, y_{i}\right) g\left(x_{i}, y_{i}\right)+t_{i} f\left(x_{i}, y_{i}\right) g_{y}\left(x_{i}, y_{i}\right)=0
\end{aligned}
$$

2. $\left(x_{i}, y_{i}\right) \rightarrow(0,0)$

3. $\left(s_{i}: t_{i}\right) \rightarrow(1: 1)$ or $(1:-1)$.

To be more general we could ask to determine the set

$$
\begin{aligned}
P(f, g):=\{ & \left\{\lim _{i \rightarrow \infty}\left(s_{i}: t_{i}\right) \mid\left(s_{i}: t_{i}\right) \in \mathbb{P}^{1}\right. \text { convergent, } \\
& \exists \text { nonzero }\left(x_{i}, y_{i}\right) \rightarrow(0,0) \text { solving } 3.83 .9 .
\end{aligned}
$$

Let us give concrete examples. Let us consider the pair $(f, g)=y^{2}-y^{3}-$ $\left.x^{3}, y^{3}+x^{2}+x^{3}\right)$. Here we have $\mu(f, g)=11$ and $\mathscr{B}(f, g)=\{0, \infty\}$ with generic Milnor number equal to one and $\mu(f)=\mu(g)=2$. If $(x, y) \neq(0,0)$ and $(s: t) \in$ $\mathbb{P}^{1} \backslash\{0, \infty\}$ solve both equations

$$
\begin{aligned}
& s f_{x} g+t f g_{x}=0 \\
& s f_{y} g+t f g_{y}=0
\end{aligned}
$$

and $(x, y)$ is sufficiently close to the origin ("sufficiently close" depending only on $f$ and $g$ not on $(s: t)$ !), then $(x, y)$ neccessarily solves

$$
f_{x} g_{y}-f_{y} g_{x}=0 .
$$

In fact, since the vector $(s, t)$ does not vanish we must have zero determinant of the matrix that occurs in the above equations: $f g\left(f_{x} g_{y}-f_{y} g_{x}\right)=0$. But if we had $f=0$, this would imply $s f_{x} g=0$ and $s f_{y} g=0$. Because $f$ and $g$ are coprime and $f$ has an isolated singularity we must have $s=0$, which contradicts our assumptions. Hence $f \neq 0$ and analogously $g \neq 0$ as was to be shown. In this example we have $f_{x} g_{y}-f_{y} g_{x}=x y(-4+6 y-6 x)$ which leaves us with two cases: either $x=0$ or $y=0$. In the first case both equations are 


\section{Milnor Number and Meromorphic Germs}

fulfilled if and only if $y^{4}(2 s+3 t)+y^{5}(-3 s-3 t)=0$ which means $y=\frac{2 s+3 t}{3 s+3 t}$. In the second case we have the solution $\left(x=-\frac{3 s+2 t}{2 s+t}, y=0\right)$. So, whenever we choose $(s: t)$ to stay away from $\{0, \infty,(-3: 2),(-2: 3)\}$, all points $(x, y) \neq(0,0)$ which solve the equations 3.11 and 3.12 will stay away from the origin in a uniform manner, i.e. there is a neighbourhood $U \subset \mathbb{C}^{2}$ of $(0,0)$ and neighbourhoods $V\left(\left(s_{i}: t_{i}\right)\right) \subset \mathbb{P}^{1}$ of each of $\left(s_{i}: t_{i}\right) \in\{0, \infty,(-3: 2),(-2: 3)\}$ so any solution $(x, y) \in U,(s: t) \in \mathbb{P}^{1} \backslash \bigcup_{i} V\left(s_{i}: t_{i}\right)$ of equations 3.11 and 3.12 will be just the origin.

If we shift the above pair $\left(f_{o}, g_{o}\right)$ to the new pair $(f, g)=\left(f_{o}+g_{o}, g_{o}\right)=$ $\left(x^{2}+y^{2}, x^{2}+x^{3}+y^{3}\right)$, then $\mu(f, g)=11$ and $\mathscr{B}(f, g)=\{(1:-1), \infty)$. The generic Milnor number is 1 and the special Milnor numbers in the pencil are $\mu(f-g)=2, \mu(g)=2$. The equation $f_{x} g_{y}-f_{y} g_{x}=0$ does not change after the shift. Inserting the solution $x=0$ into the equations 3.11 and 3.12 will give $(2 s+3 t) y^{4}=0$. Inserting the solution $y=0$ however gives $x=-2 \frac{s+t}{2 s+3 t}$. Now when $(s: t)$ approximates the value $(1:-1)$ which is one of our homotopy end values, $x$ tends to zero, i.e. in any neighbourhood of the origin there exists a nonzero solution $(x, y)$ of the set of equations with $(s: t) \approx(1:-1)$.

For the blowup approach we assume again $\mathscr{B}(f, g) \subset\{0, \infty\}$. The conjecture is $\mu(f, g)=\mu(f g)$ when the left side is finite. Now we know how the blowup-behaviour of the classical Milnor number is, namely

$$
\mu(h)-\mu(\widehat{h})=\operatorname{mult}(h) *(\operatorname{mult}(h)-1)-r(h)+1
$$

where $\widehat{h}$ is the strict transform of $h$ under a single blowup (which is a multigerm, so $\mu(\widehat{h})$ is a sum of Milnor numbers) and where $r(h)$ is the number of different tangents of the germ $h \in \mathfrak{m}_{\mathbb{C}^{2}, 0}$. If $f, g$ are given with $f(0) \cdot g(0)=0$ and $\widehat{f}, \widehat{g}$ denote their strict transforms, then we define $\mu(\widehat{f}, \widehat{g})$ as the sum of the meromorphic Milnor number of $(\widehat{f}, \widehat{g})$ at all points of the exceptional divisor $\mathbb{E}$ where at least one of $\widehat{f}$ or $\widehat{g}$ vanishes. This is the only reasonable definition since taking a point $p \in \mathbb{E}$ where $\widehat{f}(p) \neq 0, \widehat{g}(p) \neq 0$ into account would mean that we take every point $p \in \mathbb{E}$, which are of course infinitely many. In particular if $f$ has a tangent corresponding to $p \in \mathbb{E}$, but $g$ has not, then $\widehat{g}(p) \neq 0$ and so $\mu_{p}(\widehat{f}, \widehat{g})=\mu_{p}(\widehat{f})$, i.e. the classical Milnor number. The Milnor number of pairs occurs for the strict transforms only if $f$ and $g$ have a common tangent. Let $\widehat{\mathscr{B}}$ be the union of all the bifucation sets of the blowup multigerms of pairs $(\widehat{f}, \widehat{g})$. Then I guess a way to prove the conjecture 


$$
f, g \in \mathbb{C}\{x, y\}, f(0) g(0)=0, \mathscr{B}(f, g) \subset\{0, \infty\}, \mu(f, g)<\infty \Rightarrow \mu(f, g)=\mu(f g) .
$$

should consist of the following steps.

(a) Show $\widehat{\mathscr{B}}^{*} \subset \mathscr{B}^{*}$. (Recall that $\mathscr{B}^{*}:=\mathscr{B} \backslash\{0, \infty\}$.) This would make it possible to use induction on the number of blowups.

(b) Show $\mu(\widehat{f}, \widehat{g})<\infty$.

(c) Under the above assumptions we have the following blowup behaviour

$$
\mu(f, g)-\mu(\widehat{f}, \widehat{g})=\operatorname{mult}(f g) *(\operatorname{mult}(f g)-1)-r(f g)+1 .
$$

(d) Show that after sufficiently many blowups the conjecture is true.

Part $(d)$ is immediate because we can resolve the singularities of $(f: g)$ ([LW97]). Part (a) maybe also follows from this paper.

Regarding $(b)$ we have to note that it may happen that $\widehat{\mu}:=\mu(\widehat{f}, \widehat{g})$ is infinite. E.g. when $f=x$ and $g=x+h$ (with $h \in \mathfrak{m}^{2}$ reduced and $x$ does not divide $h$, see above $)$, then $\mu(\widehat{x}, \widehat{x+h})=\infty$. But $\mu(f, g)=\mu(f, f+g)$ and $\mu(\widehat{x}, \widehat{h})$ is finite! This example motivates the following

Conjecture 3.31. When we assume that the multiplicity of the pencil members $s f+t g$ changes only when $(s: t) \in\{0, \infty\}$ (in fact, there is only one member where it can change), then $\mu(\widehat{f}, \widehat{g})$ is finite!

In the above example, the multiplicity of $s \cdot f+t \cdot g=s x+t(x+h)$ changes when $(s: t)=(1:-1)$, so here $\mu(\widehat{f}, \widehat{g})$ is not neccessarily finite.

We will proceed along the lines of the proof of the blowup formula for the classical Milnor number. There are various proofs of it. For example Pham in [Pha74] proved it by using the formula $i\left(f, f_{x}\right)=\mu(f)+i(f, y)-1$ and computing the blowup in just one chart, namely the one where the derivative $f_{x}$ looks nicer. In our case we do not have such a nice formula so we have to proceed differently. We try to follow the method presented in Wall's book ([Wal04], chapter 6) but will see where it has its drawbacks. 


\section{Milnor Number and Meromorphic Germs}

Let $m:=\operatorname{mult}(f)$ and $n:=\operatorname{mult}(g)$. In the chart $(x, y) \mapsto(x y, y)$ of the blowup we compute

$$
\begin{aligned}
\widehat{f}(x, y) & =\frac{f(x y, y)}{y^{m}} \\
\widehat{g}(x, y) & =\frac{g(x y, y)}{y^{n}} \\
\partial_{x} \widehat{f}(x, y) & =\partial_{x} \frac{f(x y, y)}{y^{m}} \\
& =\frac{f_{x}(x y, y) y}{y^{m}} \\
& =\frac{f_{x}(x y, y)}{y^{m-1}} \\
\partial_{y} \widehat{f}(x, y)=\partial_{y} \frac{f(x y, y)}{y^{m}} & \\
=\frac{f_{x}(x y, y) x}{y^{m}} &
\end{aligned}
$$

It follows

$$
\begin{aligned}
\partial_{x} \widehat{f}(x, y) \cdot \widehat{g}(x, y)-\widehat{f} \cdot \partial_{x} \widehat{g}(x, y)= & \frac{\left(f_{x} g-f g_{x}\right)(x y, y)}{y^{m+n-1}} \\
\partial_{y} \widehat{f}(x, y) \cdot \widehat{g}(x, y)-\widehat{f} \cdot \partial_{y} \widehat{g}(x, y)= & \frac{\left(f_{x} g-f g_{x}\right)(x y, y) x}{y^{m+n}}+\frac{\left(f_{y} g-f g_{y}\right)(x y, y)}{y^{m+n}}+ \\
& -(m-n) \frac{(f g)(x y, y)}{y^{m+n+1}}
\end{aligned}
$$

I think the following is correct

Lemma 3.32. We have mult $\left(f_{x} g-f g_{x}\right)>=m+n-1$ and the strict inequality holds if and only if for the homogeneous parts of $f, g$ we have $f_{\text {hom }}=$ $C \cdot g_{\text {hom }} y^{m-n}$ with $C \in \mathbb{C}^{*}$. 
Let us assume that we have the equalities

$$
\begin{aligned}
& \operatorname{mult}\left(f_{x} g-f g_{x}\right)=m+n-1, \\
& \operatorname{mult}\left(f_{y} g-f g_{y}\right)=m+n-1 .
\end{aligned}
$$

Then from equation 3.15 see that for any branch $\gamma$ of $f_{x} g-f g_{x}$ the strict transform $\widehat{\gamma}$ is a "branch" of $\widehat{f}_{x} \widehat{g}-\widehat{f} \widehat{g}_{x}$. By equation 3.16 we get at any point of the above blowup chart

$$
i\left(\widehat{\gamma}, y \cdot\left(\partial_{y} \widehat{f} \cdot \widehat{g}-\widehat{f} \cdot \partial_{y} \widehat{g}\right)\right)=i\left(\widehat{\gamma}, \frac{\left(f_{y} g-f g_{y}\right)(x y, y)}{y^{m+n-1}}-(m-n) \frac{(f g)(x y, y)}{y^{m+n}}\right)
$$

Note that one summand vanishes in this computation! Now the exceptional divisor is covered by this chart except for one point. Assume that all action takes places there! (This is an assumptions on the tangents of $f_{x} g-$ $f g_{x}, f g_{y}-f_{y} g$ !) By summing up over all branches $\gamma$ this can be rewritten as

$$
\begin{aligned}
& \mu(\widehat{f}, \widehat{g})+i\left({\widehat{f_{x} g-f g}}_{x}, \mathbb{E}\right)=i\left({\widehat{f_{x} g-f g}}_{x},{\widehat{f_{y} g-f g}}_{y}-(m-n) \widehat{f g}\right), \\
& \widehat{\mu}+\operatorname{mult}\left(f_{x} g-f g_{x}\right)=i\left(\widehat{f x g-f g}_{x},{\widehat{f_{y} g-f g}}_{y}-(m-n) \widehat{f g}\right) .
\end{aligned}
$$

The right hand side is very close to

$$
\begin{aligned}
i\left(\widehat{f x}_{x-f g_{x}},{\widehat{f_{y} g-f g}}_{y}=\right. & i\left(f_{x} g-f g_{x}, f_{y} g-f g_{y}+\right. \\
& -\operatorname{mult}\left(f_{x} g-f g_{x}\right) \operatorname{mult}\left(f_{y} g-f g_{y}\right) .
\end{aligned}
$$

If it was like this we would get in the end

$$
\mu(f, g)-\mu(\widehat{f}, \widehat{g})=(m+n-1)(m+n)
$$

which is nothing but the formula $\mu(f g)-\mu(\widehat{f g})=\operatorname{mult}(f g)(\operatorname{mult}(f g)-1)+$ $r(f g)-1$ if $f$, $g$ were irreducible and shared their tangent.

However, apart from the other assumptions made during the deduction of this result, it is not true that

$$
i\left(f_{y} g-f g_{y}, x\left(f_{x} g-f g_{x}\right)-(m-n) f g\right)=i\left(f_{y} g-f g_{y}, x\left(f_{x} g-f g_{x}\right)\right) .
$$


E.g. in the example $f(x, y)=y^{3}-y^{4}-x^{3}, g(x, y)=y^{4}+x^{2}+x^{3}(\mu(f, g)=$ $\left.18, \mu(f)=4, \mu(g)=3, \mu_{\text {gen }}=3, \mathscr{B}(f, g)=\{0, \infty\}\right)$ we have $26 \neq 24$. If it was true that would be a meromorphic analogue to Wall's lemma 6.5.7.

I like to leave the discussion of the blowup approach here and discuss shortly the full conjecture. Maybe a way to prove the conjecture is to use the deformation property of $\mu$ (proposition 3.21) and to find an unfolding $\left(f_{t}, g_{t}\right)$ which has for small nonzero $t$ only points in $\left(f_{t} g_{t}=0\right)$ which have $\mu\left(f_{t}, g_{t}\right)=1$ (compare proposition 3.20). This would resemble the proof by Brieskorn ([Bri70], Appendix) that the covering degree of $\nabla f:\left(\mathbb{C}^{n}, 0\right) \rightarrow$ $\left(\mathbb{C}^{n}, 0\right)$ equals $\mathrm{rk} H^{n-1}\left(\mathrm{Mil}_{f, 0}\right)$. Can one use Suwa's result ([Suw83]$)$ on the existence of meromorphic unfoldings? 


\section{A. Appendix}

\section{A.1. Implementation in SINGULAR}

We use the computer algebra system SINGULAR ([Dec10] $)$ to compute all the ingredients of the bifurcation formula. For a given polynomial input $(f, g)$ the following code will compute

$$
i(f, g), \mu(f, g), \mu(f g), \mu_{g e n}(f, g)
$$

and $\mathscr{B}(f, g)$ together with the Milnor numbers of the special pencil members. Of course most of the data exist only if $f$ and $g$ vanish at the origin and are coprime. The implementation below follows the description of the bifurcation set due to Maugendre and Michel. Let us first show how to apply the procedure.

$$
\begin{aligned}
& >\text { LIB "bfs.lib"; } \\
& >\text { ring } \mathrm{r}=0,(\mathrm{x}, \mathrm{y}), \mathrm{ls} \text {; } \\
& >\text { poly } \mathrm{f}=\mathrm{y}^{\wedge} 4+\mathrm{x} * \mathrm{y}^{\wedge} 2+\mathrm{x}^{\wedge} 3 \text {; } \\
& >\text { poly } g=x^{\wedge} 2 * y+y^{\wedge} 2 * x \text {; } \\
& >\text { bfs }(\mathrm{f}, \mathrm{g}) \text {; } \\
& \Rightarrow \mathrm{i}(\mathrm{f}, \mathrm{g})=10 \\
& \Rightarrow \text { milnor }(\mathrm{f}, \mathrm{g})=30 \\
& \Rightarrow \text { milnor }(\mathrm{f} * \mathrm{~g})=27 \\
& \Rightarrow \text { Minmal polynomial is : } \\
& \Rightarrow(\mathrm{a} 2-2 \mathrm{a}-1) \\
& \Rightarrow \ldots \\
& \Rightarrow \mathrm{B}(\mathrm{f}, \mathrm{g})=\{(2 \mathrm{a}),(-2 \mathrm{a}+4),-1\} \\
& \Rightarrow \mathrm{mu} \quad=\{5,5,5\} \\
& \Rightarrow \text { mugen }=4
\end{aligned}
$$

The value $(s: t) \in \mathscr{B}(f, g)$ such that $s f+t g$ is a special member of the pencil is encoded as $\lambda=t / s$ if $s \neq 0$ or INF if $s=0$. I.e. the special pencil members 


\section{A. Appendix}

in the above example are $f+2 a g, f+(-2 a+4) g, f-g$. Here $a$ is a complex number annulating the minimal polynomial. Another example is

$$
\begin{aligned}
& >\mathrm{bfs}\left(\mathrm{x}^{\wedge} 2+\mathrm{y}^{\wedge} 3, \mathrm{y}^{\wedge} 2\right) ; \\
& =>\mathrm{i}(\mathrm{f}, \mathrm{g})=4 \\
& =>\operatorname{milnor}(\mathrm{f}, \mathrm{g})=\mathrm{INF} \\
& =>\operatorname{milnor}(\mathrm{f} * \mathrm{~g})=-1 \\
& =>\mathrm{B}(\mathrm{f}, \mathrm{g})=\{\mathrm{INF}, 0\} \\
& =>\operatorname{mu}=\{-1,2\} \\
& =>\operatorname{mugen}=1
\end{aligned}
$$

Here the special pencil members are $f+\infty \cdot g "=g$ and $f+0 \cdot g=f$. The first has a nonisolated singularity (therefore $\mu(f, g)=\infty$ ), while the second has Milnor number two. The generic Milnor number of the pencil is 1 .

In the implementation the Hamburger-Noether expansion of $f_{x} g_{y}-f_{y} g_{x}$ was used. One should use the printlevel= $=1$; command to turn off comments during the computation of the branches (which are indicated by ... in the above listing) or use printlevel=1; to allow for further user input during this computation. The implementation checks whether a change of ring was neccessary in order to compute the branches $\gamma$ of $f_{x} g_{y}-f_{y} g_{x}$. It uses the OrderCoeff auxiliary function to compute the order of $f \circ \gamma$. Such an expression can however be identically zero, in which case the algorithm would not terminate. However since this happens if and only if $f$ is not reduced (assuming $(f, g)=1$ ), in the very beginning we have shifted $(f, g)$ to a pair $(f+n g, n f+g)$ with a shift $n \in \mathbb{N}_{\geq 2}$ such that $f+n g$ and $n f+g$ are both reduced. Then we compute $\mathscr{B}(f+n g, n f+g)$ using the algorithm of Michel and Maugendre and extract from it the bifurcation set of the original pair $(f, g)$ using the following: If $(s: t) \in \mathscr{B}(f+n g, n f+g)$, then $(s+n t: s n+t) \in \mathscr{B}(f, g)$ and vice versa.

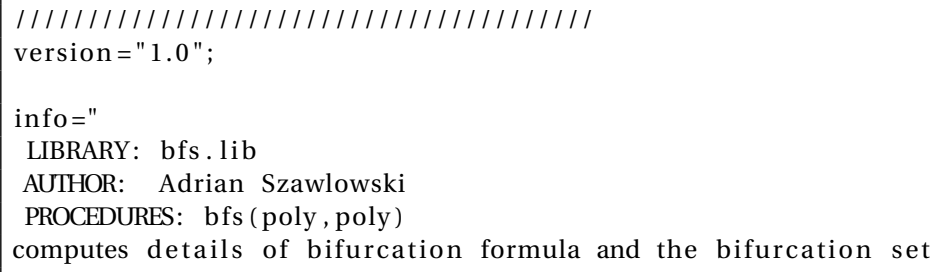




\section{A.1. Implementation in SINGULAR}

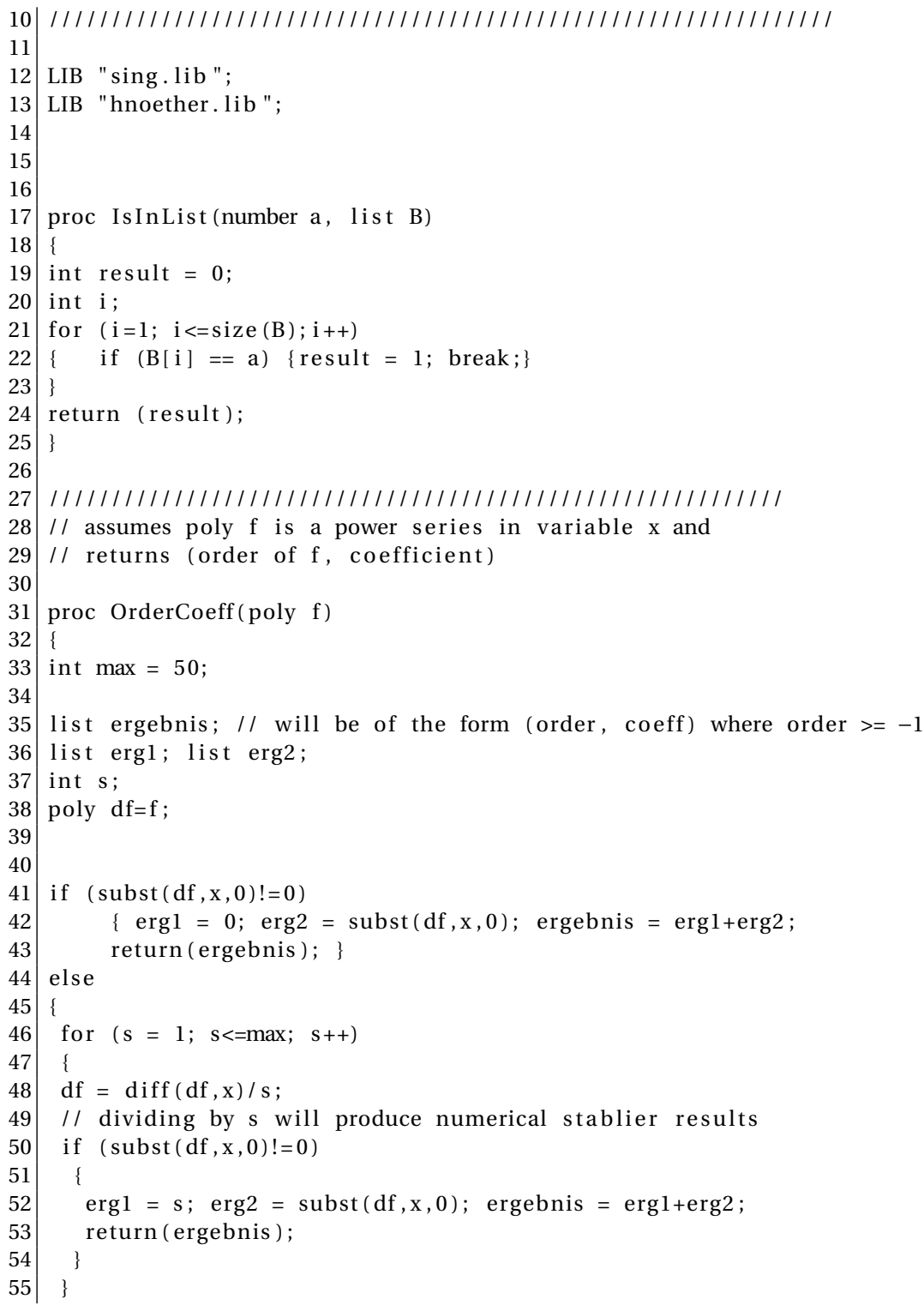




\section{A. Appendix}

56$$
69
$$

72

73\{

74 ideal $\mathrm{S}=\operatorname{diff}(\mathrm{f}, \mathrm{x}) * \mathrm{~g}-\operatorname{diff}(\mathrm{g}, \mathrm{x}) * \mathrm{f}, \operatorname{diff}(\mathrm{f}, \mathrm{y}) * \mathrm{~g}-\operatorname{diff}(\mathrm{g}, \mathrm{y}) * \mathrm{f}$;

75 int mer_milnor $=\operatorname{vdim}(\operatorname{std}(\mathrm{S}))$;

76 


\section{A.1. Implementation in SINGULAR}

102

103

104

105

106

107

108

109

110

111

112

113

114

115

116

117

118

119

120

121

122

123

124

125

126

127

128

129

130

131

132

133

134

135

136

137

138

139

140

141

142

143

144

145

146

147

ideal $\mathrm{T}=\mathrm{f}, \mathrm{g}$;

int intersection_number $=\operatorname{vdim}(\operatorname{std}(T))$;

if (intersection_number $==-1$ )

\{

"(f,g) are not coprime"; return ();

\}

else

"i $(\mathrm{f}, \mathrm{g})=$ " +string(intersection_number);

\}

if (mer_milnor! $=-1$ )

$\{" \operatorname{milnor}(\mathrm{f}, \mathrm{g})=$ "+string (mer_milnor) ;

else $\{$ "milnor $(\mathrm{f}, \mathrm{g})=\mathrm{INF} " ;\}$

$" \operatorname{milnor}(f * g)="+\operatorname{string}(\operatorname{milnor}(f * g))$;

// COMPUTATION OF THE BIFURCATION SET

$/ /$ if $\mathrm{f}$ or $\mathrm{g}$ are not reduced, then

// the algorithm by Michel, Maugendre will fail

// since $\mathrm{f} \backslash$ circ Igamma will be identically zero

// for some branch Igamma of $D=f_{-}$xg_y-f_yg_x

// so we need to shift $f$, g to reduced germs

int shift $=0$;

if $(\operatorname{milnor}(\mathrm{f})==-1$ or $\operatorname{milnor}(\mathrm{g})==-1)$

\{

for $($ shift $=2$; shift $<=100$; shift ++ )

\{

if $($ milnor $(f+\operatorname{shift} * g) !=-1$ and milnor $(\operatorname{shift} * f+g) !=-1)$

\{break;

\}

if $(\mathrm{shift}==100) \quad$ " $\mathrm{f}$ or $\mathrm{g}$ is not reduced, computation

of $\mathrm{B}(\mathrm{f}, \mathrm{g})$ not done. Raise upper bound for $<$ shift $>$ in code!"; return (); \}

poly fold = $\mathrm{f}$;

poly gold $=\mathrm{g}$;

$\mathrm{f}=$ fold + shift $*$ gold

$\mathrm{g}=\operatorname{shift} *$ fold + gold

def oldring $=$ basering;

poly $D=\operatorname{diff}(f, x) * \operatorname{diff}(g, y)-\operatorname{diff}(f, y) * \operatorname{diff}(g, x)$; 


\section{A. Appendix}

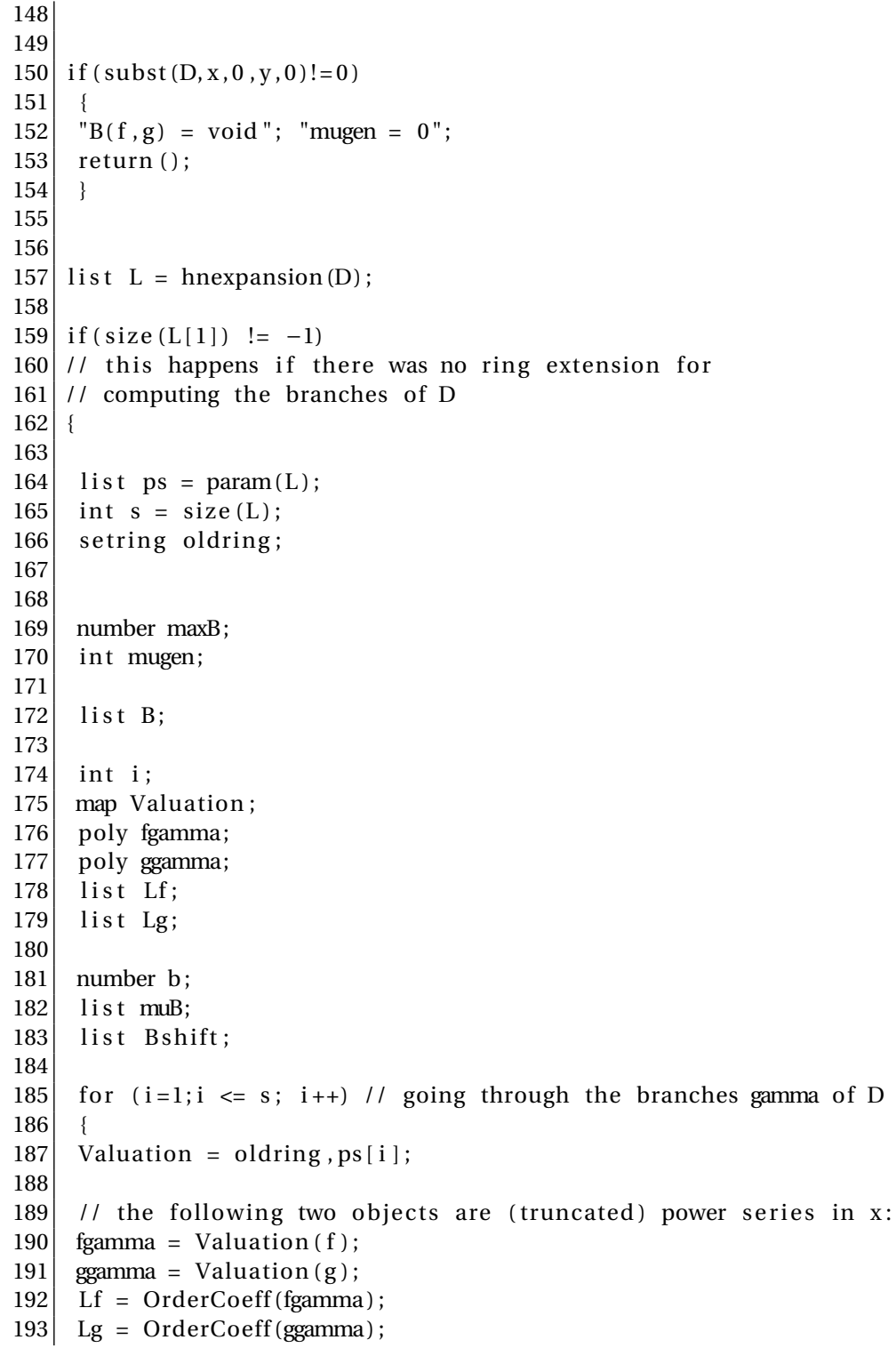




\section{A.1. Implementation in SINGULAR}

194

if $(\operatorname{Lf}[1]==-1$ or $\operatorname{Lg}[1]==-1) \quad\{\operatorname{return}() ;\}$

if $((\operatorname{Lf}[1]>\operatorname{Lg}[1]) \& \&($ IsInList $(0, \mathrm{~B}) !=1)) \quad\{\mathrm{B}=\mathrm{B}+$ list $(0) ;\}$

if $(\operatorname{Lf}[1]==\operatorname{Lg}[1]) \quad\{b=-\operatorname{number}(\operatorname{Lf}[2]) / \operatorname{number}(\operatorname{Lg}[2])$;

if (IsInList (b,B) $!=1) \quad\{\mathrm{B}=\mathrm{B}+$ list (b); $\}$

\} // for

if $(\operatorname{size}(B)==0)$

\{

mugen $=$ milnor $(\mathrm{f})$;

if $($ shift $==0$ )

\{

if $(\operatorname{milnor}(\mathrm{g})==-1)$

$\{\mathrm{B}=$ list ("INF"); "B(f,g) $=\{\mathrm{INF}\} "$;

"mu = "+string (milnor $(\mathrm{g}))$;

"mugen = "+string (mugen) $;$ \}

if (milnor $(\mathrm{g}) !=-1$ and milnor $(\mathrm{g})>$ mugen)

$\{\mathrm{B}=$ list ("INF"); "B(f, g) $=\{$ INF $\}$ ";

"mu = "+string $($ milnor $(\mathrm{g}))$;

"mugen $="+$ string $($ mugen $) ;\}$

$/ /$ in the remaining case $B$ is void,

// but this case was already treated above

// (corresp. to $\mathrm{D}(0) !=0$ )

\}

else

\{

if $(\operatorname{milnor}(g)==-1)\{$ Bshift $=1$ ist $(1 / \operatorname{number}(\operatorname{shift}))$;

$" \mathrm{~B}(\mathrm{f}, \mathrm{g})=\{"+\operatorname{string}($ Bshift $)+"\} "$;

$" m u="+\operatorname{string}($ milnor $($ fold $+(1 /$ shift $) *$ gold $))$;

"mugen $="+$ string $($ mugen $) ; \operatorname{return~}() ;\}$

if (milnor $(\mathrm{g}) !=-1$ and milnor $(\mathrm{g})>$ mugen)

$\{$ Bshift $=$ list $(1 /$ number $($ shift $))$;

$" \mathrm{~B}(\mathrm{f}, \mathrm{g})=\{$ "+string $($ Bshift $)+"\} "$;

"mu $="+\operatorname{string}($ milnor $($ fold $+(1 /$ shift $) *$ gold $)) ;$

"mugen $="+$ string $($ mugen $) ;$ return ()$;\}$

$/ /$ in the remaining case $B$ is void,

// but this case was actually already

$/ /$ treated above (corresp. to $\mathrm{D}(0) !=0$ )

\} // shift $>=2$

return (); // !

\} $/ / \operatorname{size}(\mathrm{B})==0$

if $(\operatorname{size}(\mathrm{B}) \quad !=0)$

\{

if $($ shift $==0)$

\{ 


\section{A. Appendix}

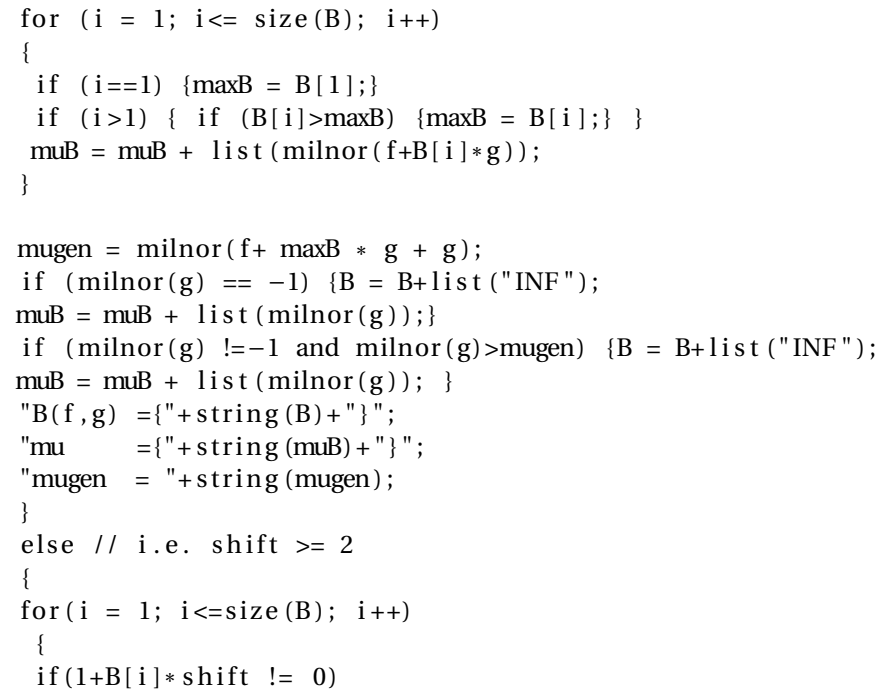


"B(f,g) $=\{"+\operatorname{string}($ B shift $)+"\} "$;

"mu $=\{"+\operatorname{string}(\mathrm{muB})+"\}$ ";

291 "mugen = "+string (mugen);

292 \} // shift $>=2$

331 for $(\mathrm{i}=1 ; \mathrm{i}<=\mathrm{s} ; \mathrm{i}++)$ 


\section{A. Appendix}

332 // going through the branches gamma of D

333 \{

334 Valuation = HNring, ps [i ] ;

// the following two objects are (truncated) power series in $\mathrm{x}$ :

fgamma = Valuation $($ hnf $)$;

ggamma = Valuation (hng);

Lf = OrderCoeff (fgamma);

Lg = OrderCoeff (ggamma);

if $(\operatorname{Lf}[1]==-1$ or $\operatorname{Lg}[1]==-1) \quad\{\operatorname{return}() ;\}$

if $((\operatorname{Lf}[1]>\operatorname{Lg}[1]) \& \&(\operatorname{IsInList}(0, \mathrm{~B}) !=1)$ )

$\{\mathrm{B}=\mathrm{B}+\mathrm{list}$ (number $(0)) ;\}$

if $(\operatorname{Lf}[1]==\operatorname{Lg}[1])\{b=-\operatorname{number}(\operatorname{Lf}[2]) / \operatorname{number}(\operatorname{Lg}[2])$;

if (IsInList $(\mathrm{b}, \mathrm{B}) \quad !=1) \quad\{\mathrm{B}=\mathrm{B}+\mathrm{list}($ number $(\mathrm{b})) ;\}\}$

\} // for

if $(\operatorname{size}(B)==0)$

\{

mugen = milnor $($ hnf $)$;

if $($ shift $==0)$

\{

if (milnor $(\mathrm{hng})==-1)$

$\{\mathrm{B}=$ list ("INF"); "B(f, g $)=\{$ INF $\} "$;

"mu = "+string (milnor (hng) );

"mugen $=$ "+string (mugen) $;$ return ()$; \quad\}$

if (milnor(hng)!=-1 and milnor(hng) > mugen)

$\{\mathrm{B}=$ list ("INF"); "B(f,g) $=\{\mathrm{INF}\}$ ";

"mu = "+string (milnor (hng) $)$;

"mugen $="+$ string (mugen) $;$ return ()$;\}$

$/ /$ in the remaining case $B$ is void

// which was already treated above

\}

else

\{

if $($ milnor $($ hng $)==-1)\{$

Bshift $=$ list $(1 /$ number $($ shift $))$;

"B(f,g) = \{ "+string (Bshift $)+"\} "$;

$" \mathrm{mu}="+\operatorname{string}($ milnor $($ hnfold $+(1 /$ shift $) *$ hngold $)) ;$

"mugen = "+string (mugen); \}

if (milnor(hng)!=-1 and milnor(hng) > mugen)

$\{$ Bshift $=$ list $(1 /$ number $($ shift $))$;

"B(f,g) = \{ "+string (Bshift $)+"\} "$;

$" \mathrm{mu}="+\operatorname{string}($ milnor $($ hnfold $+(1 /$ shift $) *$ hngold $)) ;$

"mugen = "+string (mugen) $; \quad\}$

$/ /$ in the remaining case $B$ is void, but this case 


\section{A.1. Implementation in SINGULAR}

378 // was actually already treated above

379 // (corresp . to $\mathrm{D}(0) !=0$ )

$380\}$

381 return (); // !

$382 \quad\}$

383 


\section{A. Appendix}

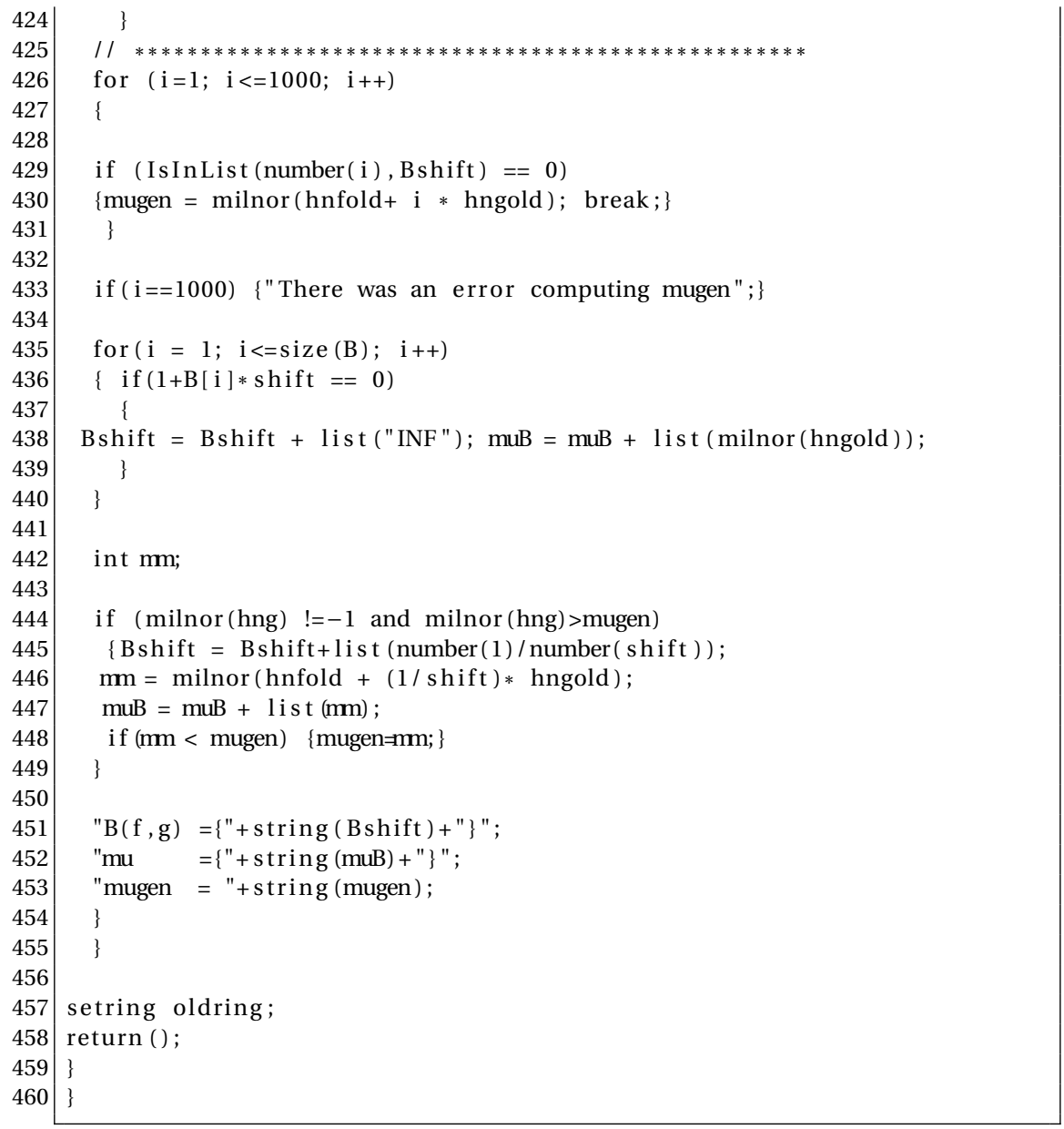




\section{Bibliography}

[AGZV85] Arnol'D, V.I. ; Gusejn-Zade, S.M. ; Varchenko, A.N.: Singularities of differentiable maps. Volume I: The classification of critical points, caustics and wave fronts. Transl. from the Russian by Ian Porteous, ed. by V. I. Arnol'd. , 1985 pg. 10]

[AGZV88] ARnol'D, V.I. ; Gusejn-Zade, S.M. ; VArChenko, A.N.: Singularities of differentiable maps. Volume II: Monodromy and asymptotics of integrals. Transl. from the Russian by Hugh Porteous. Boston, MA etc.: Birkhäuser Verlag, 1988 pg. 10

[Bar06] BARLET, Daniel: Sur certaines singularités non isolées d'hypersurfaces I. Bull. Soc. Math. France 134 (2006), no. 2, pp. 173-200 pg. 16]

[BBT03] Babelon, Oliver ; Bernard, Denis ; TAlon, Michel: Introduction to Classical Integrable Systems. Cambridge University Press, 2003 (Cambridge Monographs on Mathematical Physics) pg. 11

[BGG80] BrianÇON, Joél ; Galligo, André ; Granger, Michel: Déformations équisingulières des germes de courbes gauches réduites. Mém. Soc. Math. France 1 (1980), pp. 1-69. http://www.numdam.org/ item?id=MSMF_1980_2_1__1_0 pg. 47]

[BP07] Bodin, Arnaud ; PICHON, Anne: Meromorphic functions, bifurcation sets and fibred links. Math. Res. Lett. 14 (2007), no. 3, pp. 413-422 pg. 46

[Bri70] BRIESKORN, Egbert: Die Monodromie der isolierten Singularitäten von Hyperflächen. Manuscr. Math. 2 (1970), pp. 103-161. http:// dx.doi.org/10.1007/BF01155695 pg. 4, pg. 15, pg. 78

[BS07] BARLET, Daniel ; SAITO, Morihiko: Brieskorn modules and GaussManin systems for non-isolated hypersurface singularities. J. Lond. 
Bibliography

Math. Soc., II. Ser. 76 (2007), no. 1, pp. 211-224. http://dx.doi. org/10.1112/jlms/jdm027 pg. 16, pg. 17]

[CA00] CASAS-Alvero, Eduardo: Singularities of plane curves. London Mathematical Society Lecture Note Series. 276. Cambridge University Press., 2000 pg. 49

[Dec10] Decker, G.-M.; Pfister G.; Schönemann H. W.; Greuel G. W.; Greuel: SingULAR 3-1-1 - A computer algebra system for polynomial computations. (2010). - http://www.singular.uni-kl.de pg. 79

[Dim92] DimCA, AleXANdru: Singularities and Topology of Hypersurfaces. Springer, 1992 (Universitext) pg. 1, pg. 3, pg. 43

[Ebe01] Ebeling, Wolfgang: Funktionentheorie, Differentialtopologie und Singularitäten. Wiesbaden: Vieweg, 2001 pg. 10

[Fra78] Françoise, Jean-Pierre: Modèle local simultané d'une fonction et d'une forme de volume. Astérisque 59-60 (1978), pp. 119-130 pg. ix, pg. 15, pg. 35

[Fra82] FranÇOISE, Jean-Pierre: Réduction simultanée d'un croisement normal et d'un volume. Bol. Soc. Bras. Mat. 13 (1982), no. 1, pp. 7983. http://dx.doi.org/10.1007/BF02584736 pg. ix, pg. 17, pg. 35, pg. 43

[Gar04] GARAY, Mauricio: An isochore versal deformation theorem. Topology 43 (2004), no. 5, pp. 1081-1088. http://dx.doi.org/10.1016/ j.top. 2003.12.001 pg. 22, pg. 23

[Gar07] GARAY, Mauricio: Analytic geometry and semi-classical analysis. Proceedings of the Steklov Institute of Mathematics 259 (2007), pp. 3559. http://dx.doi.org/10.1134/S0081543807040049 pg. 28

[GLS07] Greuel, Gert-Martin ; Lossen, Christoph ; Shustin, Eugenii: Introduction to Singularities and Deformations. Springer, 2007 pg. 1, pg. 59, pg. 65

[Gre75] GreueL, Gert-Martin: Der Gauß-Manin-Zusammenhang isolierter Singularitäten von vollständigen Durchschnitten. Math. Ann. 214 
(1975), pp. 235-266. http://dx.doi.org/10.1007/BF01352108 pg. 6, pg. 16, pg. 49

[Ham71] Haмm, Helmut: Lokale topologische Eigenschaften komplexer Räume. Math. Ann. 191 (1971), pp. 235-252. http://dx.doi.org/ 10.1007/BF01578709 pg. 6

[Hir77] HironaKa, H.: Stratification and Flatness. Real and Complex Singularities (Proc. Ninth Nordic Summer School/NAVF Sympos. Math., Oslo), Sijthoff and Noordhoff, Alphen aan den Rijn, 1977, pp. 199-265 pg. 9

[Lam81] LAMотKE, Klaus: The topology of complex projective varieties after S. Lefschetz. Topology 20 (1981), pp. 15-51.http://dx.doi.org/ 10.1016/0040-9383(81)90013-6 pg. 33

[Lê73] Lê, Dũng Tráng and SAITo, Kyoji: La constance du nombre de Milnor donne des bonnes stratifications. C. R. Acad. Sc. Paris, Série A t. 277 (1973), pp. 793-795 pg. 66

[Lê74] LÊ, Dũng Tráng: Calculation of Milnor number of isolated singularity of complete intersection. Funct. Anal. Appl. 8 (1974), pp. 127-131. http://dx.doi.org/10.1007/BF01078597 pg. 49

[Lê77] Lê, Dũng Tráng: Some remarks on relative monodromy. Real and Complex Singularities (Proc. Ninth Nordic Summer School/NAVF Sympos. Math., Oslo), Sijthoff and Noordhoff, Alphen aan den Rijn, 1977, pp. 397-403 pg. 9

[Loo84] Looljenga, E. J. N: London Mathematical Society Lecture Note Series. Bd. 77: Isolated Singular Points on Complete Intersections. Cambridge University Press, 1984 pg. 5, pg. 6, pg. 7, pg. 49

[LR76] LÊ, Dũng Tráng ; RAMAnujam, Chidambaram P.: The invariance of Milnor's number implies the invariance of the topological type. Am. J. Math. 98 (1976), pp. 67-78. http://dx.doi.org/10.2307/ 2373614 pg. 51

[LT83] LÊ, Dũng Tráng ; TEISsIER, Bernard: Cycles evanescents, sections planes et conditions de Whitney. II. , 1983 pg. 5 
Bibliography

[LW97] LÊ, Dũng Tráng; WeBER, Claude: Equisingularité dans les pinceaux de germes de courbes planes et $C^{0}$-suffisance. L'Enseign. Math. 43 (1997). http://dx.doi.org/10.5169/seals-63285 pg. 46, pg. 75

[Mal74] Malgrange, Bernard: Intégrales asymptotiques et monodromie. Ann. Sci. Éc. Norm. Supér, Sér. 47 (1974), pp. 405-430. http://www. numdam.org/item?id=ASENS_1974_4_7_3_405_0 pg. 4, pg. 16

[Mar76] MARTINeT, Jean: Deploiements versels des applications différentiables et classification des applications stables. Singular. Appl. Differ., Semin. Plans-sur-Bex 1975, Lect. Notes Math. 535, 1-44 (1976)., 1976 pg. 18, pg. 22

[MH99] Melle-Hernández, Alejandro: Euler characteristic of the Milnor fibre of plane singularities. Proc. Am. Math. Soc. 127 (1999), no. 9, pp. 2653-2655. http://dx.doi.org/10.1090/ S0002-9939-99-05423-4 pg. 13

[Mil68] MiLnoR, John: Singular Points of Complex Hypersurfaces. Princeton University Press, 1968 pg. 1, pg. 6, pg. 8

[MM01] Maugendre, Hélène ; Michel, Françoise: Fibrations associées à un pinceau de courbes planes. Ann. Fac. Sci. Toulouse 10 (2001), no. 4, pp. 745-777 pg. 53

[Orl77] ORLIK, Peter: The multiplicity of a holomorphic map at an isolated critical point. , 1977 pg. 10

[Pha74] Pнам, Frédéric: Courbes discriminantes des singularités planes d'ordre 3. Asterisque 7-8 (1974) pg. 75

[Pha05] PHAM, Frédéric: Intégrales singulières. Paris: CNRS Éditions; Les Ulis: EDP Sciences, 2005 pg. 17

[Pł04] PŁosKI, ARKADIUSZ: On special values for pencils of plane curve singularities. (2004) pg. 51, pg. 54

[Seb70] Sebastiani, Marcos: Preuve d'une conjecture de Brieskorn. Manuscr. Math. 2 (1970), pp. 301-308. http://dx.doi.org/10. 1007/BF01168382 pg. 4, pg. 16. 
[Str87] Straten, Duco van: On the Betti numbers of the Milnor fibre of a certain class of hypersurface singularities. Lect. Notes Math. 1273 (1987), pp. 203-220. http://dx.doi.org/10.1007/BFb0078845 pg. 16

[Suw83] SuwA, Tatsuo: Unfoldings of meromorphic functions. Math. Ann. 262 (1983), pp. 215-224. http://dx.doi.org/10.1007/ BF01455312 pg.x, pg. 78

[Sza12a] SZAWLOWSKI, Adrian: A volume-preserving normal form for a reduced normal crossing function germ. J. of. Sing. (2012) http: //dx.doi.org/10.5427/jsing.2012.4f pg. ix, pg. 35

[Sza12b] SzAwLowsKi, Adrian: Milnor Number of Pairs and Pencils of Plane Holomorphic Germs. Sent in for publication (2012) pg. xii

[Tei76] TeIssien, Bernard: The Hunting of Invariants in the Geometry of Discriminants. Real and complex singularities. (Proc. Ninth Nordic Summer School NAVF Sympos. Math.), 1976, pp. 565-678 pg. 18, pg. 47

[Vey77] VeY, Jacques: Sur le lemme de Morse. Inventiones Mathematicae 40 (1977), pp. 1-9. http://dx.doi.org/10.1007/BF01389858 pg. 27

[Wal04] WALL, Charles Terence C.: $\quad$ Singular Points of Plane Curves. Cambridge University Press, 2004. http://dx.doi.org/10.2277/ 0521839041. pg. 72, pg. 75

[Was74] WassermanN, Gordon: Stability of unfoldings. Lecture Notes in Mathematics. Springer-Verlag, 1974 pg. 18

[Zar65a] ZARISKI, Otto: Studies in equisingularity. I: Equivalent singularities of plane algebroid curves. Am. J. Math. 87 (1965), pp. 507-536. http: //dx.doi.org/10.2307/2373019 pg. 47

[Zar65b] ZARISKI, Otto: Studies in equisingularity. II: Equisingularity in codimension 1 (and characteristic zero). Am. J. Math. 87 (1965), pp. 972-1006. http://dx.doi.org/10.2307/2373257 pg. 47 


\title{
Curriculum Vitae
}

\author{
Personal
}

Name

Adrian Szawlowski

Birth

10th Feb. 1981 in Berlin, Germany

\begin{abstract}
Academic
Oct. 2000 - Sept. 2005

Study of Mathematics at the FernUniversität Hagen
\end{abstract}

Oct. 2001 - June 2007

Study of "Technische Informatik" at the Technical University Berlin

Sept. 2005 - June 2006

Study of Mathematics at the University of Cambridge, UK

(Part III of the Mathematical Tripos)

April 2007 - April 2012

Ph.D. studies at the University of Göttingen 